\title{
Black Box Maximization of Circular Coverage*
}

\author{
C. T. Zahn, Jr.
}

\begin{abstract}
August 28, 1962
The principal problem considered is that of determining which piacement of $n$ disks of equal radius will cover as much as possible of a circular area $A$. Extensive computer experiments were performed to find the optimal arrangements and to compare the performances of several "black box" maximization methods as applied to this problem. A second version, in which $A$ is divided into subregions and each disk is regarded as contributing to the coverage of only one subregion, is also treated. Related mathematical results and questions are discussed.
\end{abstract}

\section{The Problem ${ }^{1}$}

\subsection{Description of Covering Problem}

We are given a circular area $A$ of radius $R$, centered at the origin of the $X Y$-plane, and a specified number $n$ of circular disks $C_{i}(1 \leq i \leq n)$ all having the same radius $r<R$. How should the $n$ disks be placed so that they cover as great a portion of $A$ as possible? And for this optimal placement of the disks, what is the ratio between (1) the area of the portion of $A$ covered by the disks, and (2) the total area of $A$ ?

To describe the problem more precisely we shall make the following definitions. A placement or configuration of $n$ disks is uniquely determined by specifying the $(X, Y)$ coordinates of centers of the disks. If we suppose that the $n$ disks are ordered by their indices $C_{1}, C_{2}, . ., C_{n}$, we may construct the vector $X=\left(X_{1}, Y_{1}, X_{2}, Y_{2}, \ldots . X_{n}, Y_{n}\right)$ where $\left(X_{i}, Y_{i}\right)$ denotes the center of the disk $C_{i}$. This vector $X$ with $2 n$ components completely determines a configuration of the $n$ disks with respect to the large area $A$. The configuration of figure 1 would be represented by the vector.

$$
X=(-11,6,-1,12,-3,3,-5,-4,6,1,12,7) .
$$

Now for each vector there is a uniquely determined area of that region of the plane which is within $A$ and at least one of the disks $C_{i}$. In set theoretic notation this region would be given as $\left(\bigcup_{i=1}^{n} C_{i}\right) \cap A$. In figure 1 the region we want is shaded. It should be clear that the ratio referred to above is a function $F(X)$. If we restrict the pairs $\left(X_{i}, Y_{i}\right)$ by requiring that the centers of all disks lie within $A$, then our problem is that of maximizing the function $F(X)$ over some bounded subregion of $2 n$-dimensional Euclidean space.

* Supported in part by the U.S. Army Signal Air Defense Engineering Agency. 1 The author is especially indebted to A. J. Goldman (NBS Operations Research Section) for many helpful suggestions during this research.

\subsection{Some Related Problems}

There are several problems that are closely related to our problem and which seem to be somewhat more interesting from a purely mathematical viewpoint. Since their solution for the most part involves obtaining a solution to the general problem stated in section 1.1, we shall briefly mention these others:

(a) Given disks of radius $r<R$, what is the minimum number of them required to cover $A$ completely?

(b) Given $r<R$, what is the maximum number of disks of radius $r$ that can be packed into $A$ so that there is no overlap between disks and each disk lies entirely within $A$ ? See figure 2 .

(c) Given the number $n$ of disks, what is the minimum radius $r$ for which these disks can completely cover $A$ ?

(d) Given the number $n$ of disks, what is the maximum radius $r$ consistent with packing?

(e) Given the value of $P=n r^{2}$, what values of $n$ and $r$ determine the best coverage?

Certain of the above classes of problems lend themselves to direct analytical solution. For example, problem (a) above with ${ }^{2} r=1 / 2$ can be solved briefly as follows: For complete coverage it is required that the circumference of $A$ be covered. Remembering that a regular hexagon inscribed in a circle of radius $R$ has edges of length $R$, we see that at least six disks are required to cover the circumference. But if exactly six are used then the center of $A$ is left uncovered and a seventh disk is required. It can then be shown that seven disks are in fact sufficient and the problem is solved. A similar argument can be used to show that $r=1 / 2$ is the solution to (c) when $n=7$. Problem (b) in the case $r=1 / 2$ can also be solved easily.

Probably the least trivial analytical solution in this class of problems is due to Neville [1] ${ }^{3}$, who solved problem (c) in the case $n=5$. He showed

${ }^{2}$ We assume $R=1$ for convenience; it should be noted that the ratio of coverage
depends only on $r / R$ and therefore our assumption involves no loss of generality. depends only on $r / R$ and therefore our assumption involves no loss of generality.
${ }^{3}$ Figures in brackets indicate the literature references at the end of this paper. 


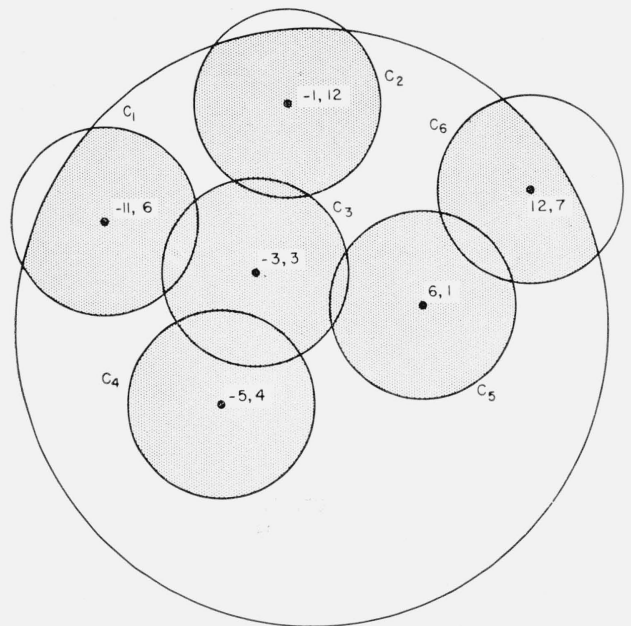

Figure 1. Coverage by six disks.

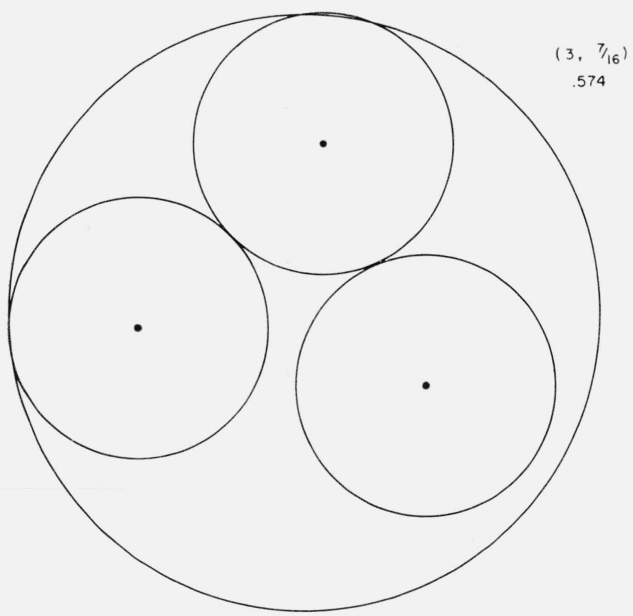

Figure 2. Loose packing of three disks.

that the minimum radius required is approximately 0.609 . It is interesting to note that the configuration of disks that achieves complete coverage with this radius does not have central symmetry. In fact the boundaries of three disks (see fig. 3) pass thru a point near the center of $A$, whereas the other two disks are considerably displaced from its center. If three of the disk-boundaries are required to pass through the center of $A$, the minimum radius needed for complete coverage increases to 0.610 , and it rises to 0.618 if all five boundaries are required to pass through the center.

Neville's result indicates that our intuitive expectations, concerning the symmetry of solutions of such problems, are not necessarily reliable. Accordingly no symmetry conditions were pre-imposed in the following work. It would be interesting to investiate further what symmetry properties can be asserted for the configuration maximizing $F(X)$, and for the configurations yielding "local maxima." It would be quite helpful, for possible subsequent

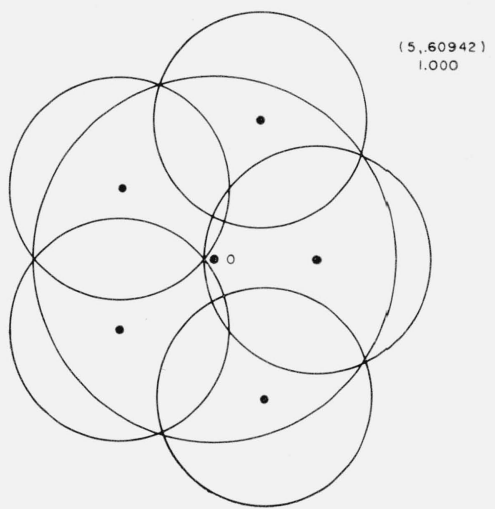

Figure 3. Neville's five disk covering.

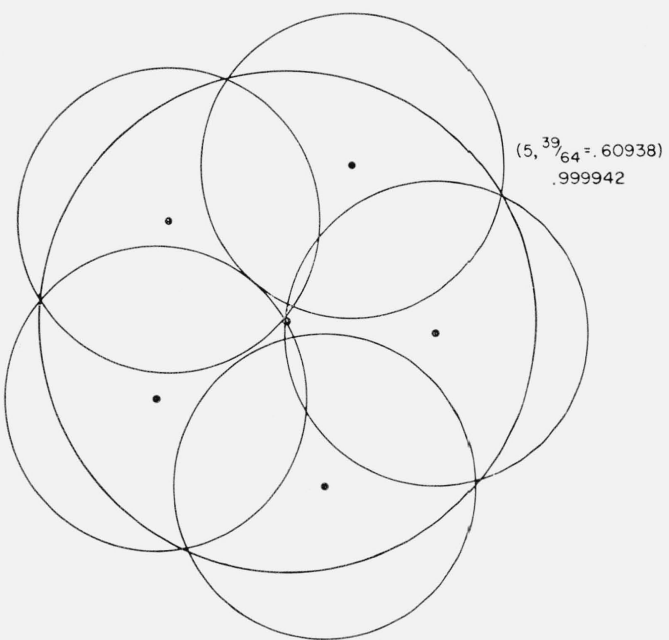

Figure 68. Computer configuration for Neville case.

research, to be assured that the maximizing configurations within certain restricted classes of symmetric patterns actually do represent at least local maxima for the covering problem. Many of the accompanying drawings (discussed later in the text) display a high degree of regularity, and the deviations from symmetry may well be due to our use of a discrete grid (see sec. 3), as well as the inevitable inexactitude of draftmanship.

\subsection{Some Related Mathematical Literature}

The several paragraphs that follow contain references to some mathematical papers that are relevant to the problems discussed in this paper. We hope the interest of these topics will be an adequate compensation for the lumpiness of their presentation.

An interesting result concerning coverage by disks of equal size is the following theorem of $R$. Kerschner [2]. 
If $N(r)$ is the smallest number of disks of radius $r$ needed to cover a plane set of area $A$, then

$$
\lim _{r \rightarrow 0} \pi r^{2} N(r)=2 \pi \sqrt{3} A / 9
$$

This formula suggests the possibility of using

$$
N_{0}(r)=2 \pi \sqrt{3} / 9 r^{2}
$$

as an estimate for the minimum number of disks of radius $r$ required to cover completely a large disk whose area is $\pi$ (i.e., whose radius is $R=1$ ). For example, $N_{0}(3 / 8)=9$ to the nearest integer whereas 10 disks at this radius can be made to cover 99 percent of the area $A$, as indicated in table 1 .

The following theorem of Verblunsky [3] relates to how fast the convergence is in Kerschner's result:

There is a number $c>1 / 2$ such that, for all small enough $r$

$$
N(r)-\left(2 \sqrt{3} / 9 r^{2}\right)>(2 \sqrt{3} c / 9 r)
$$

where $N(r)$ is the least number of disks of radius $r$ required to cover a square of area 1 . This means that the approximation $N_{0}(r)$ suggested above converges at best on the order of $(1 / r)$. In the absence of other knowledge, however, this might be used to get some idea of what sort of coverage might reasonably be expected with a particular pair $(n, r)$. The Verblunsky result applies to coverage of a square but it seems quite likely that the convergence is similar for the circular coverage problem.

The following result [4] establishes a relationship between the problems of packing and covering. If $r_{1}$ and $r_{2}$ denote respectively the maximum $r$ for packing and the minimum $r$ for covering with $n$ disks then

$$
3 r_{2}>4 r_{1}
$$

This is true whenever the region to be covered or packed is convex. We can use this result to get a lower bound on $r_{2}$ if we know $r_{1}$, and vice versa.

The two-dimensional case of a more general theorem of D. Gale [5] implies that any plane set of diameter 2 can be covered by three properly chosen sets each of diameter $\leq \sqrt{3}$. The author also points out that no three sets each of diameter $<\sqrt{3}$ will cover a disk of diameter 2 . This essentially solves problem (c) for the case $n=3$ and indicates that a disk is the "hardest" set to cover among sets of equal diameter.

An interesting related problem arises if the $n$ disks $C_{i}$ are "thrown down" independently at random, i.e., with their centers uniformly distributed subject only to the condition that they overlap the circular area $A$. Here $F(X)$ becomes a random variable whose mean is an appropriate reference point in deciding which values of $F(X)$ might be considered high ones. The value of such a reference point is enhanced if one also has at hand the standard deviation $\sigma$ of $F(X)$, which can be obtained from

$$
\sigma_{2}=M_{2}-\left(M_{1}\right)^{2}
$$

where $M_{2}$ is the second movement of $F(X)$ and $M_{1}$ is the mean (i.e., the first moment).

Such problems of "random coverage" have been treated in the technical literature. The basic theorem on this subject, due to A. Kolmogoroff [6] can be stated for our purposes as follows: Suppose one has a probability distribution over a specified class of sets $S$ in $m$-dimensional Euclidean space. ${ }^{4}$ Then the measure ${ }^{5} \mu(S)$ of a set $S$ is a random variable. If points of the Euclidean space are denoted $x=\left(x_{1}, \ldots, x_{m}\right)$ and $y=\left(y_{1}, \ldots, y_{m}\right)$, then the mean of $\mu(S)$ is given by ${ }^{6}$

$$
M_{1}=\int \operatorname{Prob}(x \text { is in } S) d x_{1} \ldots d x_{m},
$$

its second moment by

$$
\begin{aligned}
& M_{2}=\iint \operatorname{Prob}(x \text { is in } S \text { and } y \text { is in } S) \\
& \qquad d x_{1} \ldots d x_{m} d y_{1} \ldots d y_{m},
\end{aligned}
$$

and similarly for higher moments.

This theorem was rediscovered by H. E. Robbins [7], who used it to study the one-dimensional analog of our problem, i.e., random coverage of a linear interval by smaller intervals. He calculated $M_{1}$ and $M_{2}$ for this case, and observed that his formula for $M_{1}$ remains valid for the two-dimensional ("circles") case that concerns us here. Subsequently J. Bronowski and J. Neyman [8] treated the random coverage of a fixed rectangle by smaller rectangles with sides parallel to those of the fixed one. Robbins [9] solved the $m$-dimensional generalization of the problem for rectangles, and also treated random coverage of a rectangle by circular disks. L. A. Santalo [10] treated random coverage of an $m$ dimensional rectangle by spheres, ${ }^{7}$ and also the coverage of a (two-dimensional) rectangle by rectangles of random orientation. He also solved the problem of random coverage of a sphere in $m$ dimensional space by smaller spheres, which for $m=2$ is the problem that concerns us.

Of the many formulas derived in these papers, only two will be cited here. Both refer to the area of a circular region $A$ of radius $R=1$, which is covered by the union of $n$ circular disks $C_{i}$ of radius $r<1$, whose centers are independently chosen and uniformly distributed over a disk of radius $1+r$ concentric with $A$.

The first formula, due to Robbins, gives the mean ${ }^{8}$ of this "random covered area" as

$$
M_{1}=\pi\left[1-\left(1-\left(\frac{r}{1+r}\right)^{2}\right)^{n}\right]
$$

\footnotetext{
${ }^{4}$ In our case $m=2$ and the sets $S$ are non-empty intersections of the circular disk $A$ with the union of $n$ circular disks of radius $r$.

" "Measure" is here a generic term which means "length" in one-dimensional situations, "area" in two dimensions, and "volume" in three.

6 The integral formally extends over the entire Euclidean space, but in most applications the integrand is zero outside some bounded region.

7 Note that a "sphere" is just a linear interval in one-dimensional situations, and is a circular disk in two dimensions.

8 See table 0 for values of $M_{1} / \pi$ pertinent to this study.
} 
this must be divided by the area $\pi$ of $A$ to obtain the mean of the ratio $F(X)$. The second formula, due to Santalo, gives the corresponding variance as

$$
\begin{gathered}
\sigma^{2}=2 \pi \int_{0}^{2 r} \frac{\left[1-2 \pi r^{2}-2 r^{2} \operatorname{arc} \cos (t / 2 r)+\frac{1}{2} t\left(4 r^{2}-t^{2}\right)^{1 / 2}\right.}{\pi(1+r)^{2}} \\
\left.+(2 \arccos (t / 2))-\frac{1}{2} t\left(4-t^{2}\right)^{1 / 2}\right] t d t \\
+\left(1-\frac{2 r^{2}}{(1+r)^{2}}\right)^{n}\left\{\pi^{2}-2 \pi\left(2\left(2 r^{2}-1\right) \arccos r\right.\right. \\
-3 r\left(1-r^{2}\right)^{1 / 2}+\pi+2 r\left(1-r^{2}\right)^{3 / 2} \\
-\arcsin r)\}-\pi^{2}\left(1-\left(\frac{r}{1+r}\right)^{2}\right)^{2 n}
\end{gathered}
$$

this must be divided by $\pi^{2}$ to obtain the variance of $F(X)$.

\section{Attempts at Analytical Solution}

\subsection{Formula for Maximand}

Returning to the main problem presented in section 1.1, we shall describe some attempts that were made to obtain an analytical solution. Problems

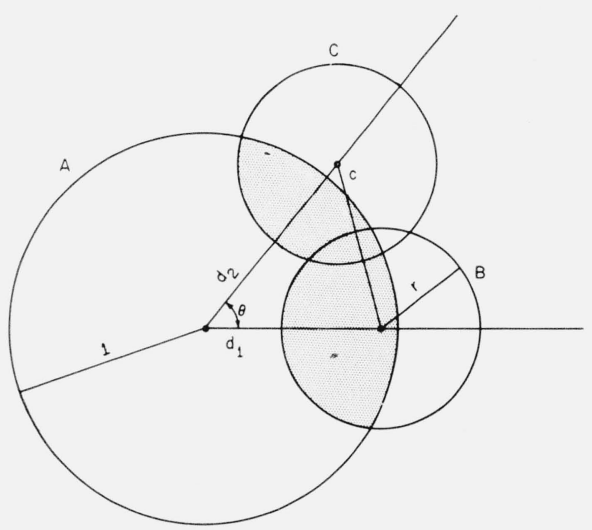

Figure 4. Parameters in the two disk case.

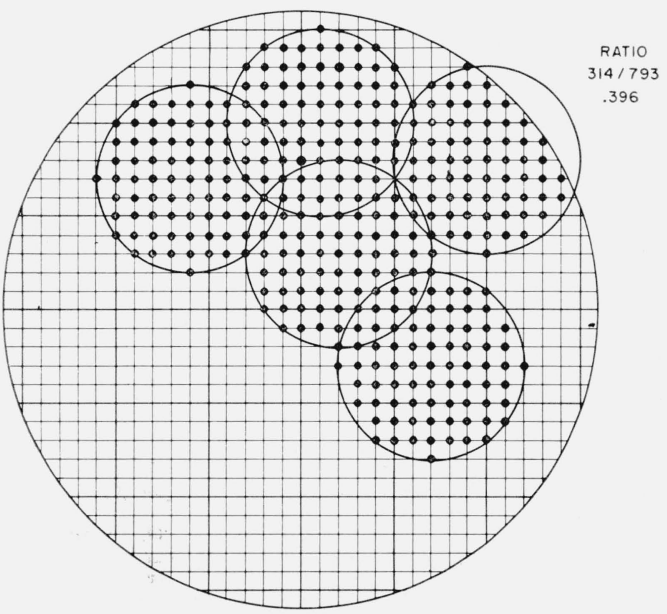

Figure 5. Approximating the coverage. involving the maximization of a function of several variables can usually be handled by calculus if the function $F(X)$ can be written as an expression involving the components of $X$ and familiar functions of them. The first step in any attempt at an analytical solution to our problem is to obtain some "formula" for that portion of the area of $A$ that is covered by the configuration $X=\left(X_{1}, Y_{1}, \ldots, X_{n}, Y_{n}\right)$.

\subsection{The Two-Disk Problem}

An initial attempt was made to derive a "formula" for the area covered by two disks of radius $r<R=1$. The parameters describing the placement (see fig. 4) of the disks were

(1) $d_{1}$ and $d_{2}$, the distances from the center of $A$ to the centers of disks $C_{1}$ and $C_{2}$, respectively,

(2) $\theta$, the angle between these two distances $(\theta \leq \pi)$. It was thought that with these parameters in place of $\left(X_{1}, Y_{1}, X_{2}, Y_{2}\right)$, it would be easier to obtain the formula desired. ${ }^{9}$ It was found that a single formula could not be obtained for the area covered but an algorithm was devised which uses no less than eight

${ }^{9}$ Every configuration of $n$ dis's can actually be specified by only $(2 n-1)$ variables, by arbitrarily setting $X_{1}=0$. This involves no loss in generality, for if we are given a configuration where $X_{1} \neq 0$, then a rotation of the coordinates can be performed so as to make $X_{1}=0$. Such a rotation will not alter the coverage of the configuration.

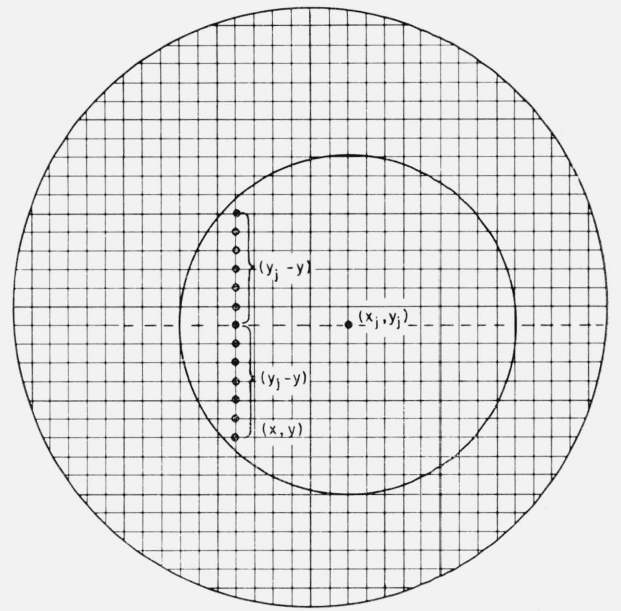

Figure 6. Reflection short-cut.

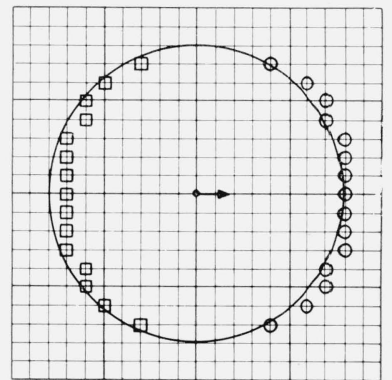

Figure 7. Points to be scanned. 
"formulas," depending on certain geometric properties of the covering configuration. ${ }^{10}$

\subsection{Abandonment of Analytical Methods}

The impossibility of obtaining any reasonable "formula" for the function we are trying to maximize in the relatively trivial case $n=2$ seems to indicate the futility of the analytical approach especially when $n$ is larger. On this sad note the general analytical approach was abandoned and another method of a somewhat experimental nature, using high-speed electronic computers, was adopted.

\section{Black Box Maximization}

\subsection{General Description}

Procedures collectively known as "Black Box Maximization" have been used recently to search for the maximum value of a function [11-14]. They are employed when the following conditions exist:

(1) It is required to maximize a certain function $F(X)$, where $X$ ranges over some finite set of objects $S$.

(2) For each individual $X$ it is possible to calculate $F(X)$, but there is no neat analytical expression for $F(X)$.

(3) The function $F$ has some sort of continuity which makes it possible to define, for each $X$ in $S$, a subset $N(X)$ of the points of $S$ in such a way that the value of $F$ at $X$ differs from the value of $F$ at any point of $N(X)$ by a small amount. This set $N(X)$ is called the set of neighbors of $X$.

In one such method, the search for the maximum of $F$ over $S$ proceeds as follows: Pick some point $X_{1}$ in $S$ as a starting point and calculate $F\left(X_{1}\right)$. Then determine the points belonging to $N\left(X_{1}\right)$ and compute $F$ for each of these points. Among the members of $N\left(X_{1}\right)$ select one that yields the highest function value. If this point $X_{2}$ has a higher function value than $X_{1}$, then repeat the process with $X_{2}$ replacing $X_{1}$. However, if $F\left(X_{2}\right) \leq F\left(X_{1}\right)$, then $X_{1}$ is a relative maximum of $F$ and the iteration terminates.

The method we have just described is known as the method of "steepest ascent," since in selecting the new point $X_{2}$ we picked that member of $N\left(X_{1}\right)$ with the greatest function value. Two other methods which might be employed are worthy of notice. If we select as $X_{2}$, that neighbor of $X_{1}$ which has the smallest value of $F$ among those whose function values are greater than $F\left(X_{1}\right)$, this is known as the method of "slowest ascent" or "least positive ascent." This method derives its rationale by analogy with the case of searching for the absolute maximum of a function of 2 variables where the function can be considered as a surface in 3 space with hills and valleys representing extrema. There is some intuitive reason [11] for surmising that following a "river bed" may lead to a higher peak than a "steep climb" would obtain. The third method, known as the "first positive ascent," derives its

10 See appendixes 1 and 2. value from the fact that the selection of the new point $X_{2}$ at each stage generally takes less computation than for the other two methods, and thus saves valuable time when an electronic computer is being used to solve the problem. In this method the members of $N\left(X_{1}\right)$ are arbitrarily ordered into a sequence $N_{1}, N_{2}, \ldots, N_{p} . F\left(N_{1}\right)$ is computed and if it exceeds $F\left(X_{1}\right)$ then $N_{1}$ is selected as $X_{2}$. If $F\left(N_{1}\right) \leq F\left(X_{1}\right)$ the computation is repeated with $N_{2}$ and so on until one of the neighbors of $X_{1}$ is selected or all the neighbors are exhausted. If the latter occurs, then no points of $N\left(X_{1}\right)$ have a higher function value than $X_{1}$, so $X_{1}$ is a relative maximum of $F$. There is no known way of selecting one of these methods as best, even given certain characteristics of the maximand. Gleason [11] presents interesting statistics comparing the "steepest ascent" with the "slowest ascent" for one particular problem, but no general comparison seems possible short of numerous experiments.

\subsection{Application to the Coverage Problem}

We observed in section 2.2 that the function $F(X)$ we want to maximize could not be expressed in any neat formula. In fact, where more than two disks are involved, the construction of an algorithm to calculate the function would probably be too difficult and time-consuming to be worthwhile. What we need, first of all, is an approximation for $F(X)$. We assume that the circular area $A$ is centered at the origin and its radius $R$ is a positive integer. Furthermore, the radius $r$ of the disks is also a positive integer. ${ }^{11}$ The points of the plane $(p, q)$ where both $p$ and $q$ are integers are called gridpoints and the area of $A$ is approximated by the number of grid-points $n_{1}$ which lie inside the boundary of $A$. We shall also require that each pair $\left(\dot{X}_{i}, Y_{i}\right)$ determining the center of a disk $C_{i}$ be a grid-point. Our estimate for the portion of $A$ covered by the disks is the number of grid-points $n_{2}$ that lie inside $A$ and at least one of the disks (see fig. 5). The ratio $F(X)$ is approximated by the quotient $n_{2} / n_{1}$.

The problem is now to maximize $F(X)$ where $F$ is given by the approximation, over all vectors $X$ such that the components are integers $j$ in the range $-R \leq j \leq R$. In this form the problem satisfies conditions (1) and (2) of section 3.1. To satisfy the third condition we must specify the neighborhood $N(X)$ for each vector $X$ in the domain of $F$. We shall define $N(X)$ to be all those vectors in the domain of $F$ which can be derived from $X$ by adding \pm 1 to exactly one component of $X$. This means that each $X$ has $4 n$ neighbors except in the boundary situations (some component of $X$ is $\pm R$ ) where it has less.

The problem as now formulated can be submitted to the methods of maximization described above.

${ }^{11}$ Since the function $F(X)$ is a ratio, the value of $F$ is not changed if $R, r$, and the vector $X$ are multiplied by a constant factor. 
Some rather slight deviations from the general method were employed because of certain peculiarities of our problem, but for the most part these methods were the techniques that were programed for use on a computer.

\subsection{Drawbacks of the Methods}

At this point we should acknowledge several rather sobering facts about our method. The black box maximization methods described (steepest, slowest, and first positive ascent) all find a point ${ }^{12}$ which is a relative or "local" maximum of the function; if one were certain beforehand that the function has only one such "local" maximum, there would be no further problem. Unfortunately, this is usually not the case because one is dealing primarily with functions whose behavior is generally unknown. The best way to increase the probability of hitting on the true "global" maximum seems to be to repeat the search many times with random initial points and different schemes of ascent. Secondly, the discretizing of the problem which was effected in order to be able to approximate the function has introduced some error into the numerical results. Although theoretically the mesh can be refined ${ }^{13}$ to obtain any accuracy desired, the limitations set by time and the size of computer memory make it impossible to refine the mesh indefinitely.

\subsection{A Related Topic}

A topic related to the methods discussed in section 3 of this paper is that of maximizing an "unknown" function whose every evaluation requires physical experimentation and so, besides being costly, involves experimental errors. Since the classical paper of Box and Wilson [15] appeared, much work has been published in statistical journals on the design of efficient explorations schemes for such "response surfaces"; we mention here only a paper of Box and Hunter [16] and those by Brooks [17].

\section{The Computer Program}

\subsection{Specializations and Subroutines}

The technique we have described was programed ${ }^{14}$ and coded in FORTRAN and SAP for use on an IBM 704 electronic computer. The code has been debugged and a large body of data has been collected. There are several things which did not appear in the preceding description of the method but were necessary additions or at least were clearly indicated.

The procedure for choosing an initial "vector" $X$ to begin a search was left unspecified in the foregoing.

\footnotetext{
12 "Point" is used here to mean an element in a vector space; i.e., a vector. 13 The mesh is refined by multiplying the appropriate variables by a constant factor; this does not sound like refinement but it amounts to the same.

14 See appendix 3 ; the FORTRAN program there reproduced is for the Cycling First Positive Gradient described in section 4.2, paragraph 5.
}

As the computer code was written, the selection is made as follows :

(1) Using "steepest ascent," the initial $X$ is chosen by randomly generating a certain number of vectors and selecting the one that achieves the highest function value.

(2) Using either "slowest ascent" or "first positive ascent," the initial $X$ is selected by a single random generation.

(3) Using any of the methods, the initial $X$ may be read into the computer as an input variable.

Another variation on the general method that was present in the program was refinement of the mesh. In section 3.2 we required that $R$ and $r$ be positive integers and that the centers of the disks be gridpoints (i.e., $X_{i}$ and $Y_{i}$ must be integers for all $i$ ). The value of $R$ is further limited by the program to the powers of 2 , and each time a relative maximum is achieved with some value of $R$ the mesh is refined by doubling $R, r$, and $X$. This is effectively the same as if we had actually refined the mesh of grid-points by constructing new lines halfway between those that already define our grid-points. After this refinement has been carried out, the search is continued until a relative maximum is found. The mesh is then refined once again and so on until the mesh is as fine as we desire. Since the fineness of the mesh is indicated by the value of $R$, we specify as an input to the program the maximum value of $R$ indicating the final mesh size. The reason for this successive refinement is that if the search is begun with a coarse mesh, the bulk of the searching process can be done in a lesser amount of time; this is because the amount of computation depends very strongly on the number of grid-points, as might be expected.

There are ten subroutines that are called for by the main program; a listing of these and a brief description of each follows:

MK2T - computes and stores in memory a table of the squares of all positive integers less than 1,000.

XBAR - randomly generates $n$ pairs of coordinates $\left(X_{i}, Y_{i}\right)$ where $X_{i}^{2}+Y_{i}^{2}<R^{2}$ with $X_{i}$ and $Y_{i}$ integers.

SUMX - computes for the current $X$ and mesh size $R$, the number of grid-points which lie inside the large circle $A$ and at least one of the disks. This number is called NSUM.

RAT - computes the total number of grid-points in $A$ and divides NSUM by this number to get RATIO, our $F(X)$.

VECTOR - for each particular value of $r$, selects those points near the boundary of a disk which should be scanned to determine whether some movement of a disk represents a gain or loss in NSUM. ${ }^{18}$

NEAR - for each disk $C_{j}$ a determination is made of those disks near enough to $C_{j}$ so that they might overlap $C_{j}$ if $C_{j}$ were moved by one unit in some direction. For example, in figure 1 it should be quite clear that disk $C_{1}$ need not be considered when we are interested in knowing what effect small changes in the position of $C_{5}$ have on the value of NSUM.

\footnotetext{
15 See section 4.2, paragraph (4), for details
} 
SCAN 1-computes the change in NSUM due to one of the four possible changes in the position of a particular disk $C_{j}$, when the disk is entirely within the boundary of $A$ and would still be so even after one of the four changes in its position.

SCAN 2-performs the same computation as SCAN 1 when the disk $C_{j}$ is partially outside $A$ or might be after a single move.

XNEW-changes the "vector" $X_{1}$ to the new "vector" $X_{2}$ indicated by the search procedure.

REFINE - multiplies the variables $R, r$, and $X$ by two, thereby effecting the mesh refinement.

For each of the different methods of ascent a separate program has been written. Each program uses the ten subroutines to do the bulk of the work.

\subsection{Shortcuts in the Program}

Some special techniques and shortcuts were used in the program and subroutines; they were devised partly to save time in computation and partly as a result of certain peculiarities of the specific problem to be solved.

(1) The restrictions that were imposed on the problem in section 3.2 may seem somewhat artificial. As a matter of fact, there was a definite reason for recasting the problem in such a way that almost all the variables involved are integers. The computer for which the program was written (IBM 704) has separate sets of instructions to deal with integer variables and noninteger variables, and the time required to add two integers is two machine cycles ${ }^{16}$ where the (floating) addition of two nonintegers takes from 7 to 11 cycles. It was thought that this difference would affect greatly the total time required for a run.

(2) The computation of the table of integer squares up to 1000 which is done by the subroutine MK2T was inserted into the program to save time also. A single multiplication takes 20 machine cycles on the IBM 704 whereas looking up the square of an integer from a table stored in the memory takes only four cycles.

(3) In the calculation of NSUM, the total number of grid-points that are covered by the configuration $X$, it is possible to simplify the computation procedure by the following shortcut:

The calculation of NSUM is performed by scanning all the grid-points on some vertical line $\mathrm{x}=\mathrm{k}$, an integer. The lowest grid-point on the line that is also inside $A$ is the first to be scanned. For each successive grid-point proceeding in the direction of positive $y$ values, a decision is made as to whether or not the point falls in one of the disks $C_{j}$. Suppose that a certain grid-point $(X, Y)$ is determined to be inside a disk $C_{j}$ with center $\left(X_{j}, Y_{j}\right)$, as in figure 6 . Rather than continuing on to the next adjacent point, we calculate the quantity $\left(Y_{j}-Y\right)$ and draw the conclusion that all points between $(X, Y)$ and

$$
\left(X, Y+2\left(Y_{j}-Y\right)\right)=\left(X, 2 Y_{j}-Y\right)
$$

${ }^{16}$ A machine cycle in the IBM 704 requires $12 \mu$ sec. are also inside $C_{j}$. The number of points to be scanned has thus been substantially reduced.

(4) The subroutine NEAR (see sec. 4.1) saves some time in computation by selecting from the set of disks, those that have no effect on small movements of a certain disk $C_{j}$. For example, if the program were about to determine what changes occur in NSUM as a result of moving disk $C_{6}$ of figure 1 to any of the four positions possible, it would not be necessary to consider disks $C_{1}, C_{2}, C_{3}$, or $C_{4}$ in making this calculation. When the total number of disks is larger this shortcut in the computation should be quite effective.

(5) The general method calls for computing the function value $F(X)$ for all vectors $X$ that are "neighbors" of the current vector $X_{1}$. From our definition of neighbor we can see that a neighbor of $X_{1}$ corresponds to a configuration derived from that of $X_{1}$ by moving some $C_{j}$ one mesh-unit in one of four directions. Fortunately it is not necessary to compute $F(X)$ at all neighboring points. Whichever method of ascent is to be used, the important value to be computed is $\Delta F=F(X)-F\left(X_{1}\right)$, and for each $X$ this can be computed without computing either $F(X)$ or $F\left(X_{1}\right)$. We ask the questions: How many mesh-points that were not covered by $X_{1}$ are covered by $X$ ? How many points that were covered by $\dot{X}_{1}$ are not covered by $X$ ? The answer to the first question tells us how many points have been gained and the answer to the second, how many have been lost. The difference between these two is the net gain in covered points due to changing $X_{1}$ to $X$. In figure 7 the two separate sets of meshpoints indicate respectively the set of points that could either be "lost" or "gained" by moving the disk to the right. Furthermore, for any direction, two similar sets of mesh-points can be selected to scan in computing $\Delta F$.

(6) Using the terminology of the preceding section (5), only points that are within $A$ are candidates for classification as gains or losses. Therefore, if the disk $C_{j}$ being scanned is inside $A$ by at least a meshunit (that is, all points of $C_{j}$ are inside $A$ and no closer than one mesh-unit from the boundary of $A$ ), then all the points on the periphery of $C_{j}$ are inside $A$ and qualify as possible gains or losses. In this case SCAN 1 is used to compute $\Delta F$. However if $C_{j}$ overlaps $A$, we must determine for each meshpoint being scanned whether it lies inside $A$. In this case SCAN 2 is used.

\subsection{Inputs and Outputs}

Very few input variables are required for the program. A list of the most important and a description of each follows: ${ }^{17}$

N7 - the number of separate cases to be done.

J7-this variable indicates whether or not the initial "vector" is to be read in as an input.

$\mathrm{KO}$ - Initial radius of large area $A$ (this must be some power of 2 ).

17 Integer variables in a FORTRAN code must be designated by a symbol beginning with one of the letters $\mathrm{I}, \mathrm{J}, \mathrm{K}, \mathrm{L}, \mathrm{M}, \mathrm{N}$. This is why the radius $R$ of the disk $A$ to be covered is denoted by $\mathrm{KO}$. 
$\mathrm{NC}$ - The number of covering disks.

$\mathrm{N}$ - The number of random selections of a "vector" to choose the initial vector.

KBIG-This number represents the finest mesh to be used.

JXPO Satisfies the equation $2^{\mathrm{JXPO}}=\mathrm{KO}$.

IRO - Initial radius of covering disks (satisfies IRO $<\mathrm{KO}$ ).

$\mathrm{N} 4$ - Number of times each case is to be repeated with different initial "vectors."

As far as outputs are concerned, a fairly readable format has been devised. Initially, a general description of the case to be done and the important inputs are printed out. Next the initial "vector" is printed out with its function value RA'TIO. Each move that occurs is printed out and the final configuration at each mesh size is recorded with its RA'TIO. The total number of moves that have been made is also printed out when the final configuration has been attained.

\section{Results of Computer Runs}

\subsection{Description of Cases Studied}

A systematic series of runs was completed of cases involving between 2 and 10 disks where the ratio $r / R$ took on interesting values ${ }^{18}$ between 0 and 1 . Table 1 gives the fraction of $A$ that was covered by the best configuration found during the search for the global maximum. Diagrams of these configurations can be found in figures 8 to 44 . These numbers are accurate to about \pm 0.002 . A detailed explanation of the convergence properties of the approximation is given in section 6 .

\subsection{Closeness of Relative Maxima}

In the cases where two, three, or four disks were used to cover, the final configurations were all global maxima. In the other cases there were as many as

\footnotetext{
18 A case is not interesting if complete coverage is possible with a smaller number of disks, or if all disks can be placed inside $A$ so as not to overlap.
}

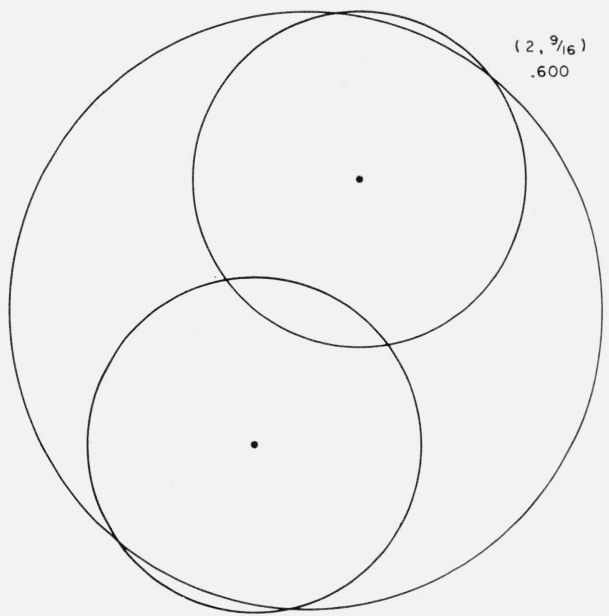

Figure 8. Case $(2,9 / 16)$. five different local maxima found. Two configurations were considered to be different if the configurations were geometrically unlike. For example, the configurations of figures 45 and 46 are considered to be the same whereas both are different from that of figure 47 .

Diagrams of the local maxima that were found in several cases are depicted in figures 45 to 60 . The value of RATIO is included with each configuration. It should be pointed out that the three best con-

TaвLE $0 . \quad$ Mean coverage ratio* with case $(\mathrm{n}, \mathrm{r})$

\begin{tabular}{|c|c|c|c|c|c|c|c|c|c|}
\hline$n$ & 2 & 3 & 4 & 5 & 6 & 7 & 8 & 9 & 10 \\
\hline $5 / 16$ & & & & & & & 0.373 & 0.409 & 0.442 \\
\hline $3 / 8$ & & & & 0.321 & 0.371 & 0.418 & .461 & .501 & .538 \\
\hline $\begin{array}{l}116 \\
1 / 2\end{array}$ & & 0.298 & $\begin{array}{r}0.322 \\
.376\end{array}$ & $\begin{array}{l}.385 \\
.445\end{array}$ & $\begin{array}{l}.442 \\
.507\end{array}$ & $\begin{array}{l}.494 \\
.562\end{array}$ & $\begin{array}{l}.541 \\
.610\end{array}$ & $\begin{array}{r}.583 \\
\end{array}$ & $\begin{array}{r}.622 \\
\end{array}$ \\
\hline 916 & 0.242 & .341 & .426 & .500 & .565 & & & & \\
\hline $5 / 8$ & .274 & .381 & .473 & .551 & & & & & \\
\hline $11_{16}$ & .304 & .420 & .516 & 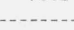 & & & $\cdots$ & $-\cdots$ & - . - \\
\hline $3 / 4$ & .334 & .456 & .556 & & & & & & \\
\hline $13 / 16$ & .362 & .490 & & & & & & & \\
\hline $7 / 8$ & .388 & .521 & & & & & & $\ldots$ & - \\
\hline $1^{15}{ }_{16}^{16}$ & .414 & - & & & & & & & -. \\
\hline 1 & .431 & & & & & & & & \\
\hline
\end{tabular}

*The centers of the disks are randomly chosen from a uniform distribution on the circular disk of radius $(1+r)$

The entry in the table is the expected value of the ratio of coverage where $n$ disks of radius $r$ are randomly placed as described.

This table contains the mean coverage for the same cases for which table 1 gives the maximum coverage.

TABLE 1. Maximum ratio of coverage with case $(\mathrm{n}, \mathrm{r})$

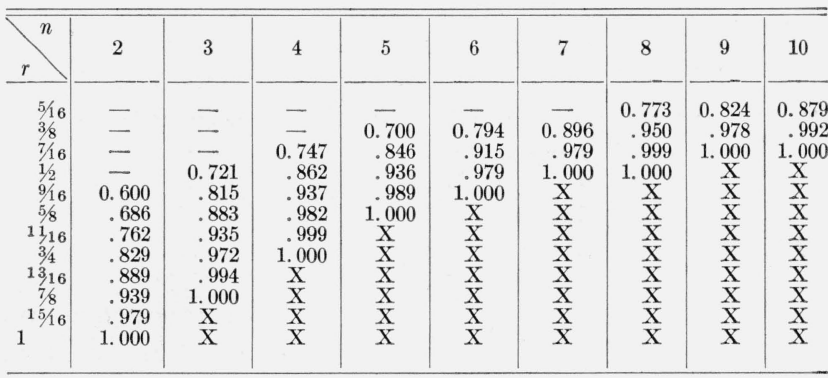

- indicates all disks can be packed into large circle.

$\mathrm{X}$ indicates total coverage is possible.

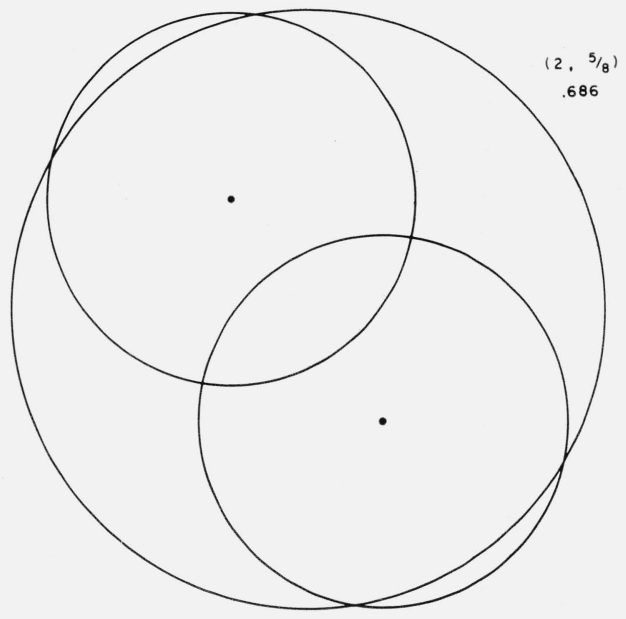

Figure 9. Case (2,5/8). 


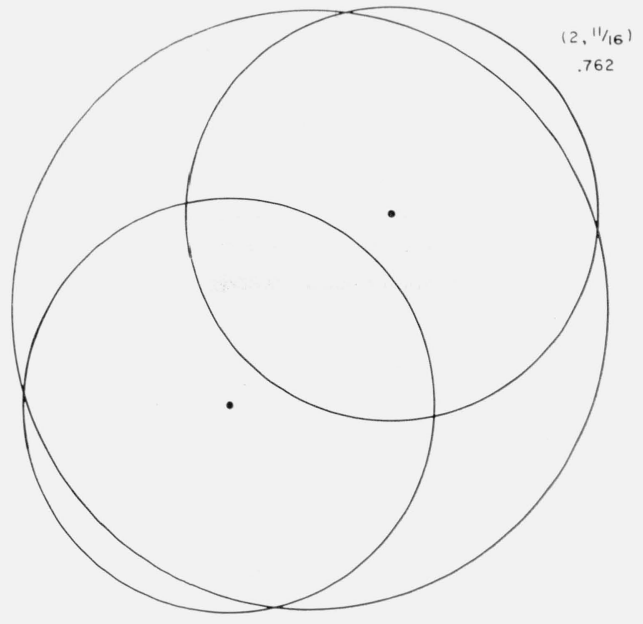

Figure 10. Case $(2,11 / 16)$.

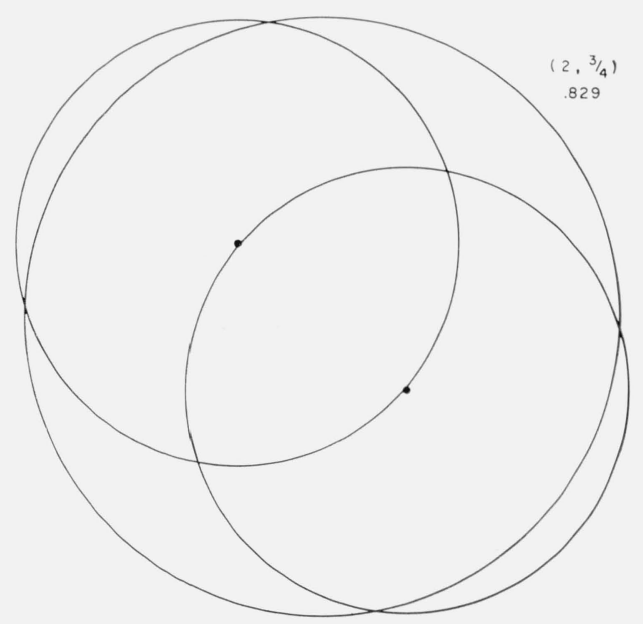

Figure 11. Case (2,3/4).

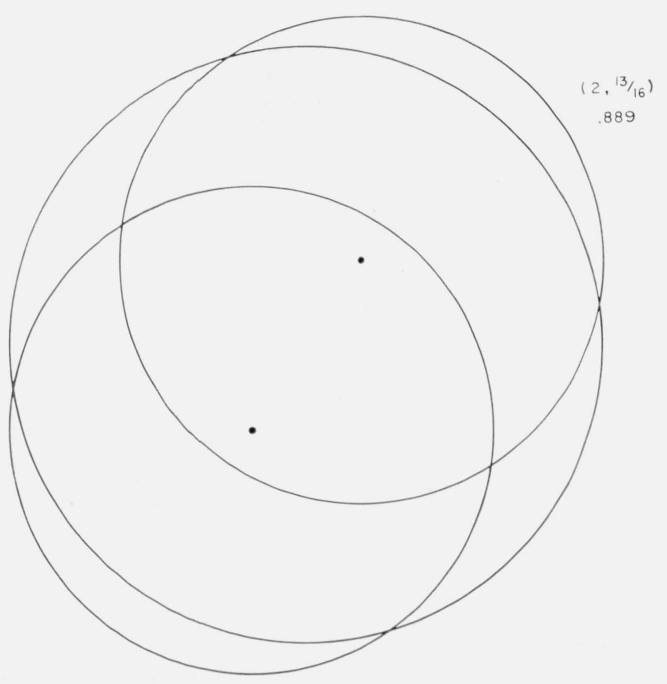

Figure 12. Case $(2,13 / 16)$.

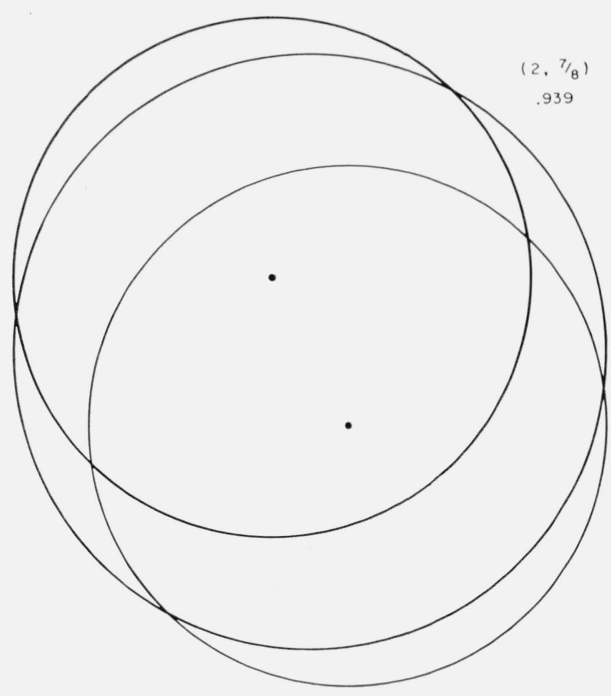

Figure 13. Case (2, $7 / 8)$.

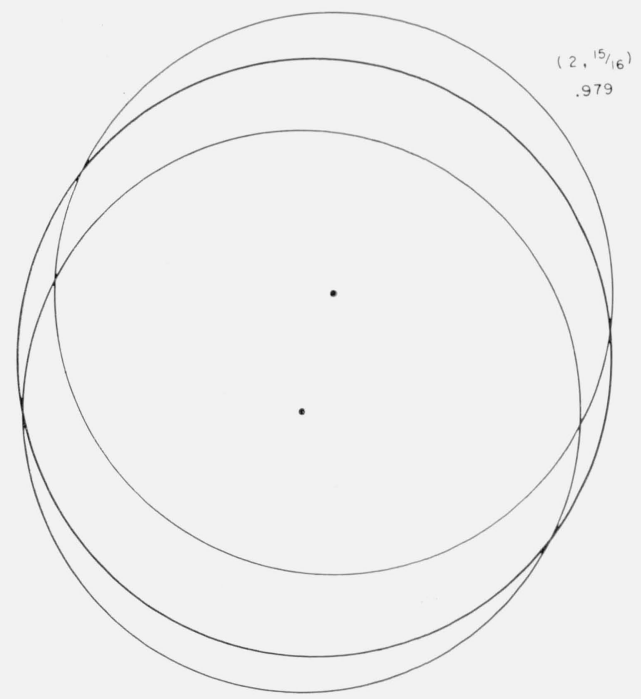

Figure 14. Case (2,15/16).

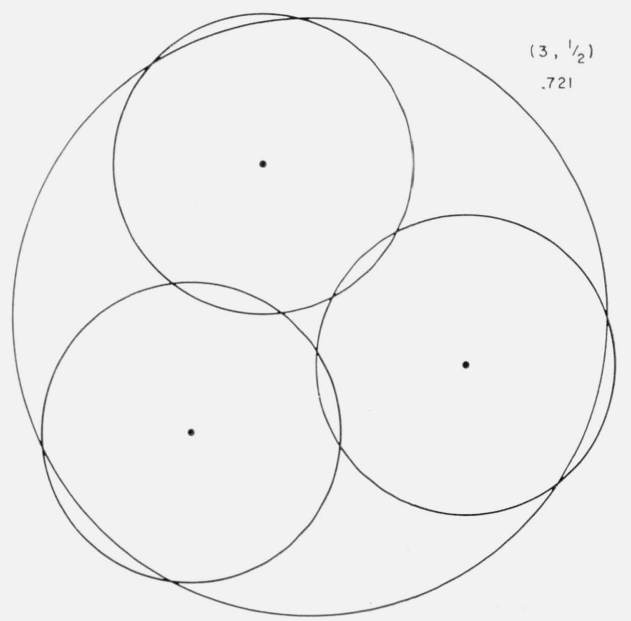

Figure 15. Case (3,1/2). 


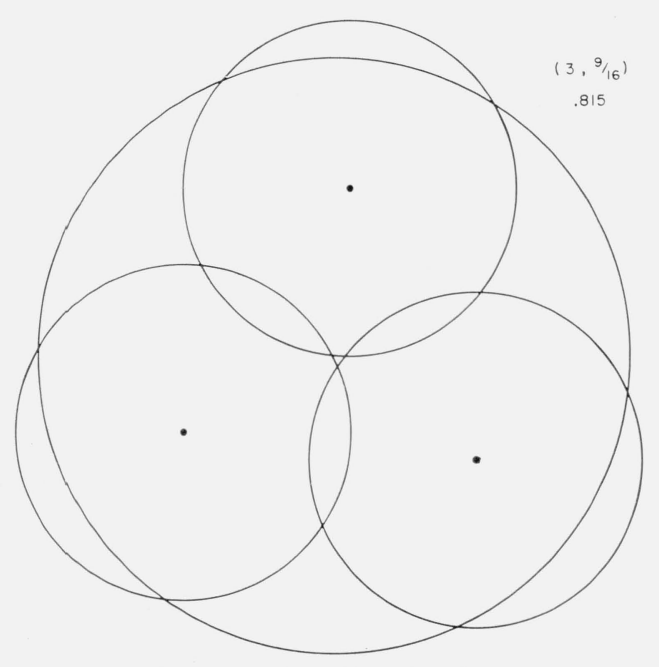

Figure 16. Case (3,9/16).

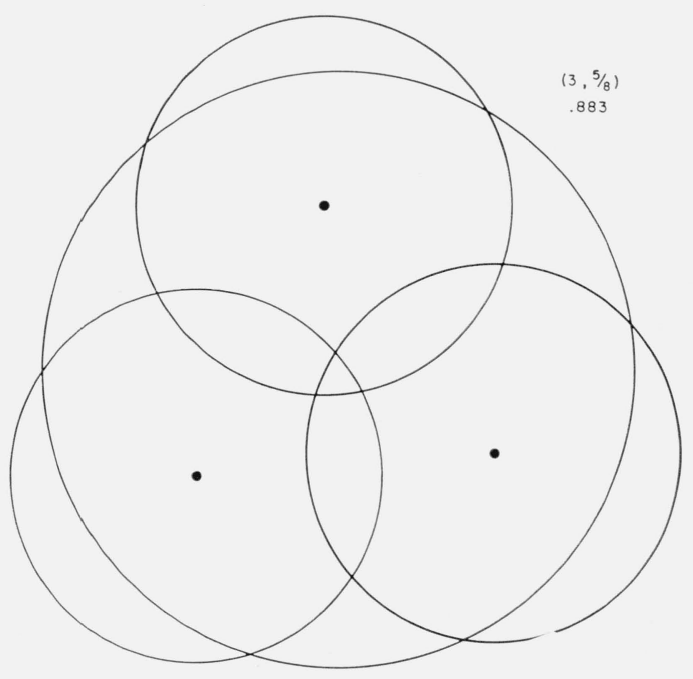

Figure 17. Case $(3,5 / 8)$.

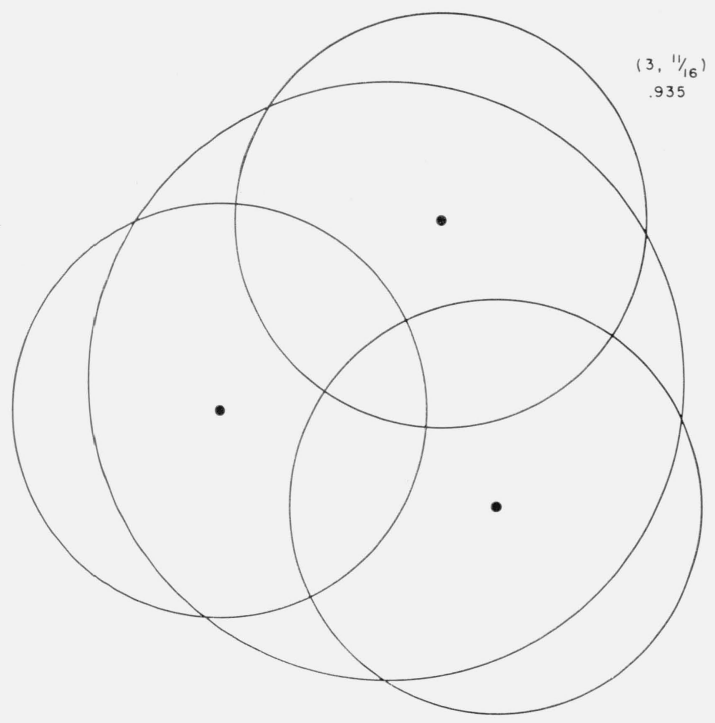

Figure 18. Case $(3,11 / 16)$.

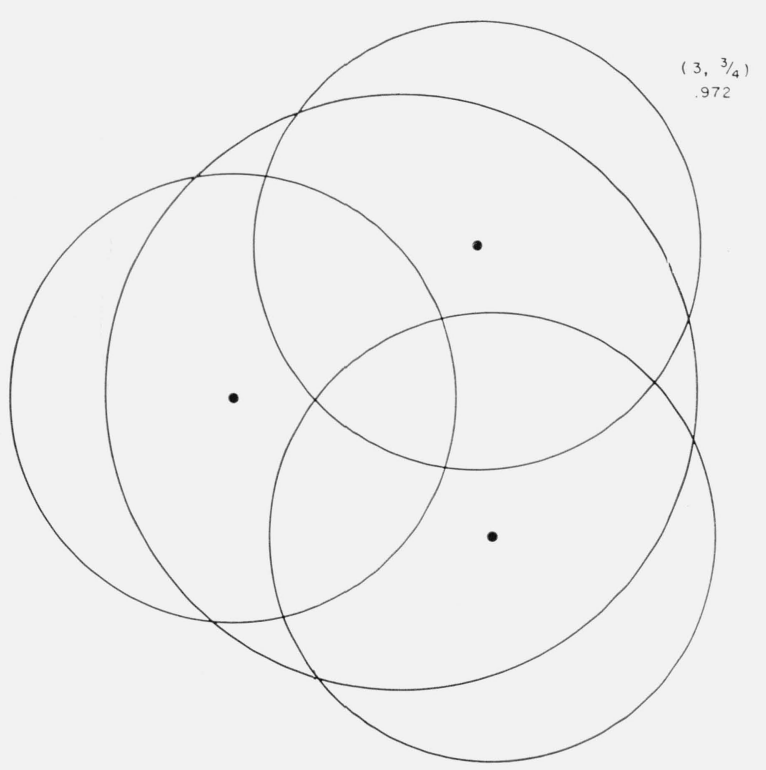

Figure 19. Case $(3,3 / 4)$.

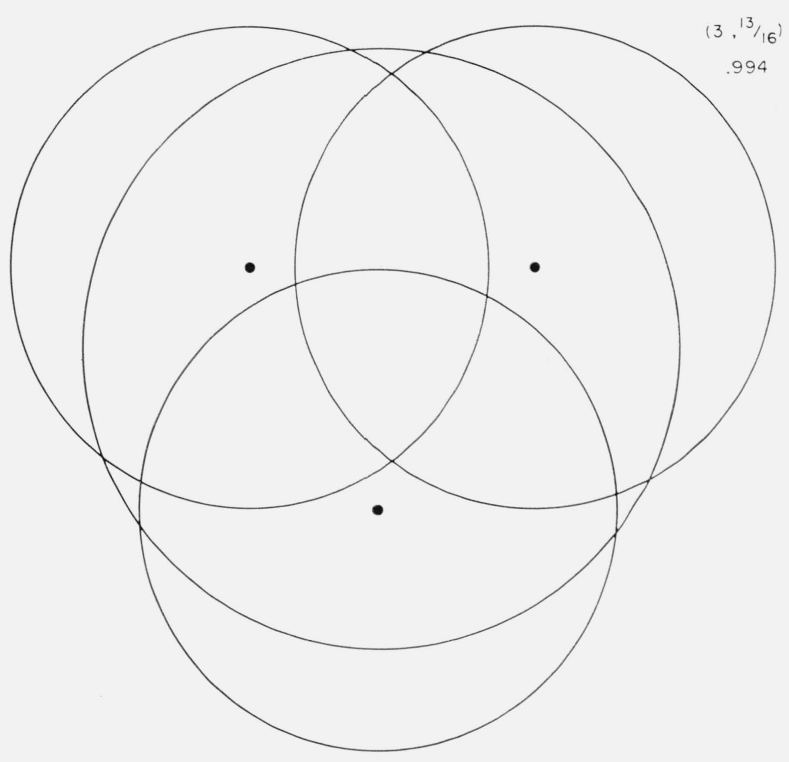

Figure 20. Case $(3,13 / 16)$.

figuration for the case ${ }^{19}(6,1 / 2)$, figures 48 and 49 , differ in function values by at most 0.006 . In case $(10,3 / 8)$, figures 43 and 44 , the two best local maxima differ by less than 0.002 , and in case $(9,5 / 16)$, figures 57 and 58, with the mesh refined to 256 , the two local maxima differ by less than 0.0002 . In the latter case the ratios must be considered as indistinguishable due to the error of the approximation at this mesh. ${ }^{20}$

19 The following notation specifies the situation $n=6, r=1 / 2$ (and $R=1$ ). This method of denoting cases will be used consistently in the rest of the paper.

20 See section 6 .

190 


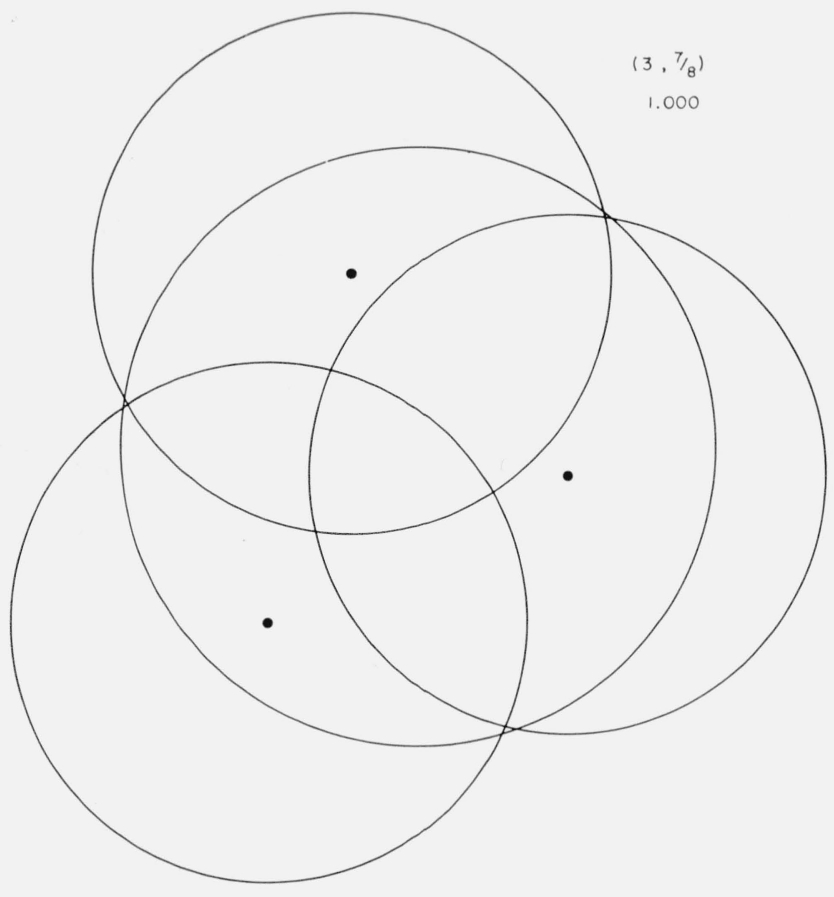

Figure 21. Case $(3, \gamma / 8)$.

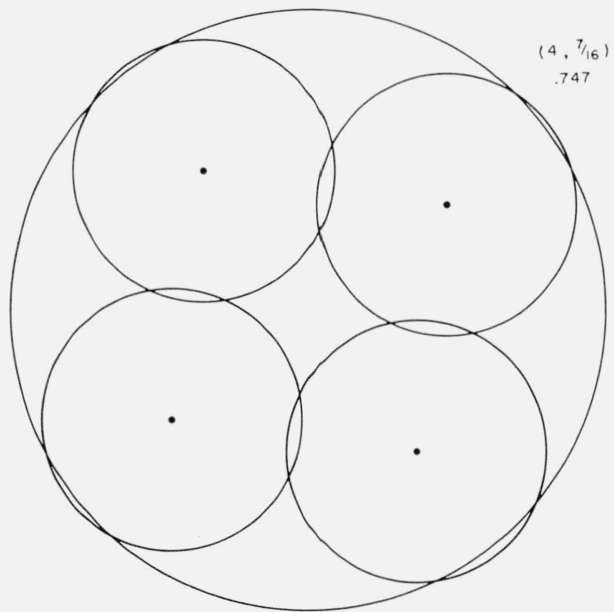

Figure 22. Case $(4,7 / 16)$.

In general it was found that the differences between the local maxima in each case were on the order of one or two percent of the total coverage. The largest such difference that was observed was in case $(8,3 / 8)$ where peaks of height 0.905 and 0.947 were found, constituting a difference of about 4 percent.

\subsection{Details for the Six-Disk Case}

A more detailed study was made of the local maxima achieved with six covering disks at radii of $5 / 16,3 / 8,7 / 16,1 / 2$, and $9 / 16$. The case $(6,5 / 16)$ is one that we termed "not interesting" in section 5.1. because

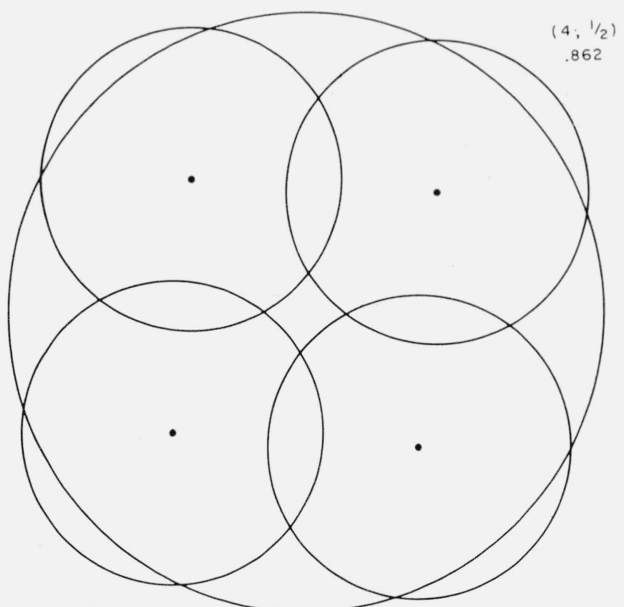

Figure 23. Case (4,1/2).

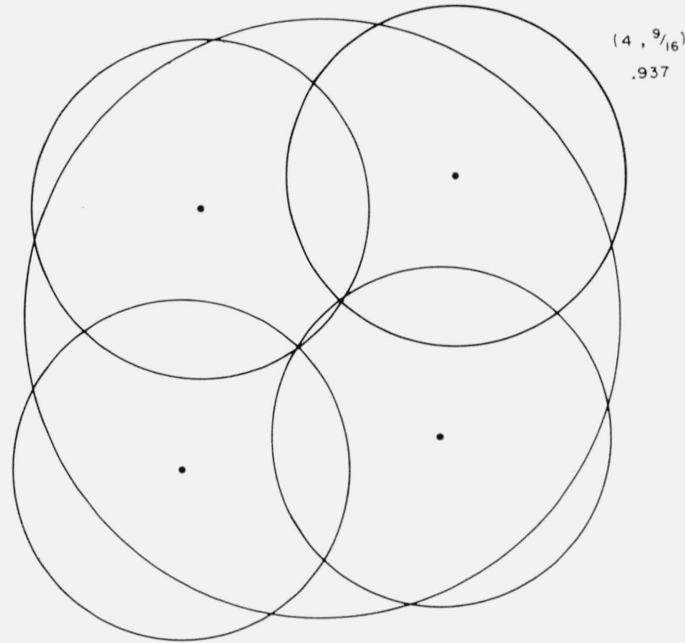

Figure 24. Case $(4,9 / 16)$.

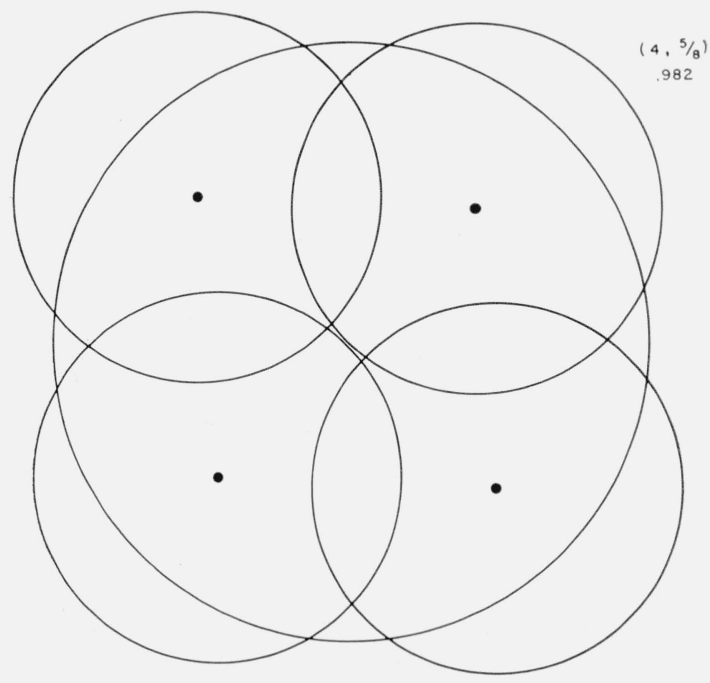

Figure 25. Case $(4,5 / 8)$. 


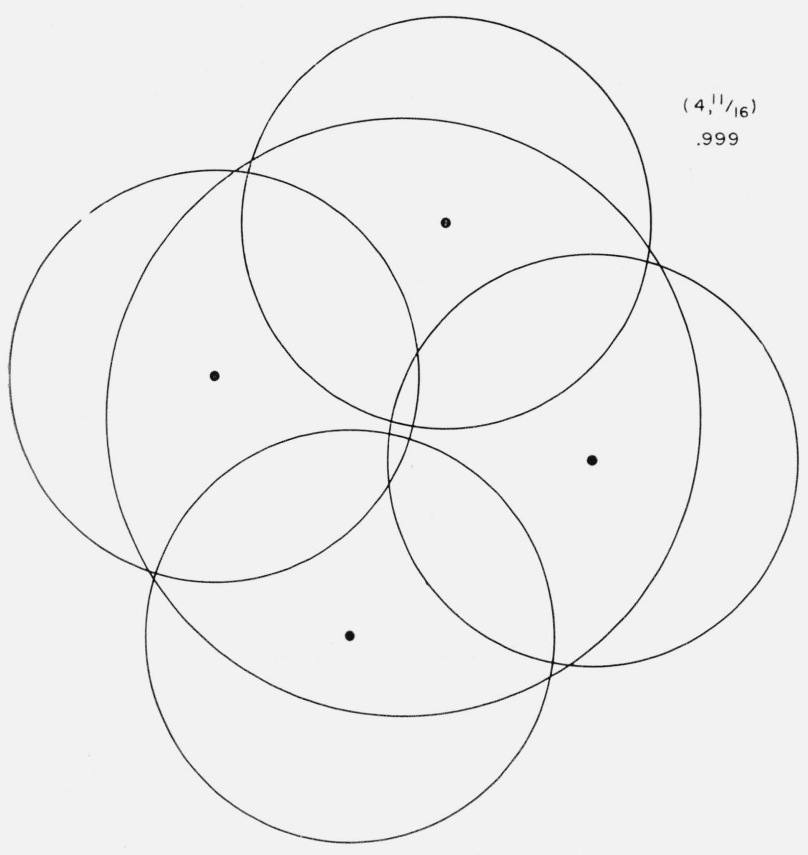

Figure 26. Case $(4,11 / 16)$.

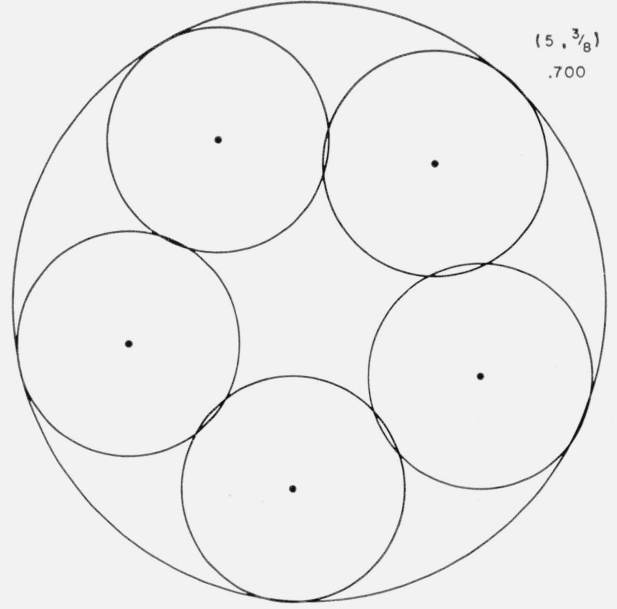

Figure 27. Case $(5,3 / 8)$.

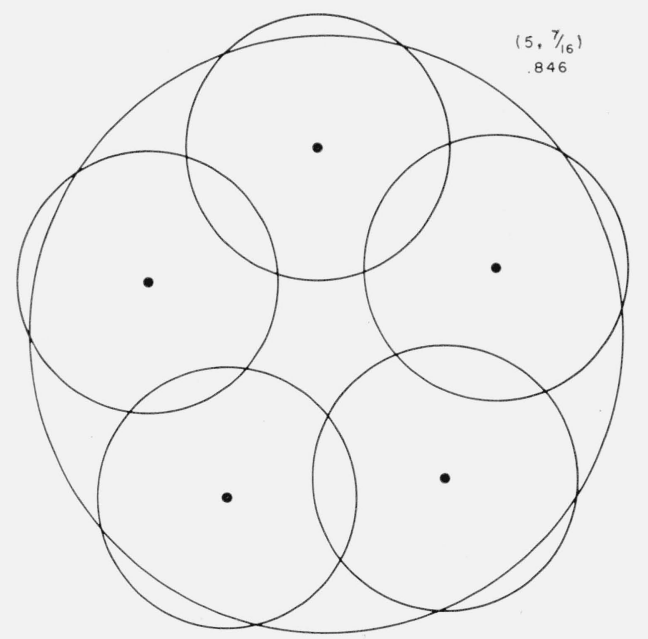

Figure 28. Case $(5, \tilde{\gamma} / 16)$.

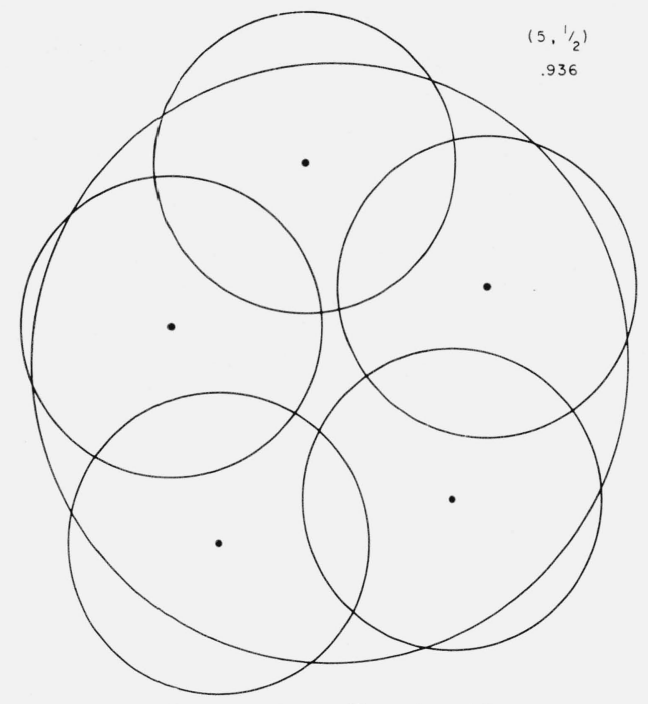

Figure 29. Case (5,1/2).

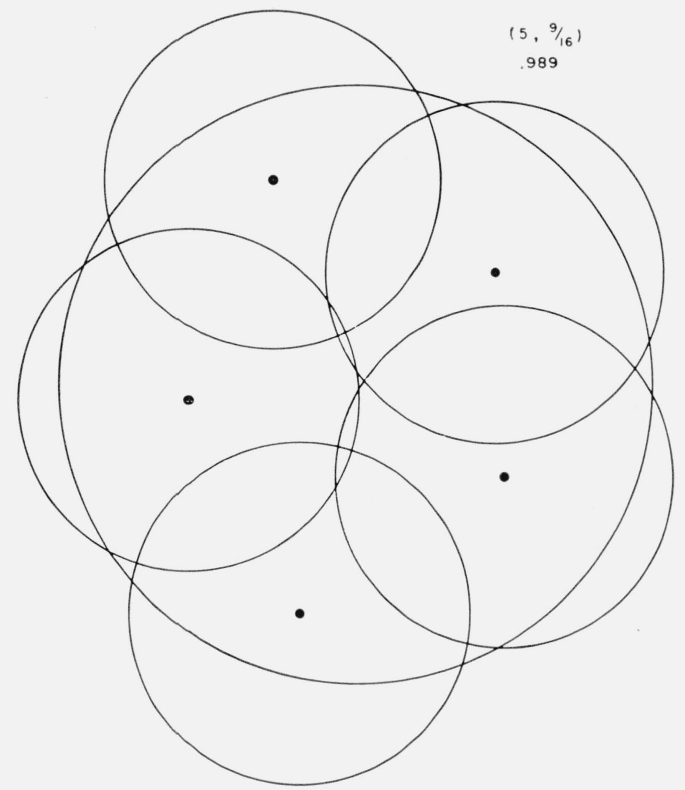

Figure 30. Case (5,9/16).

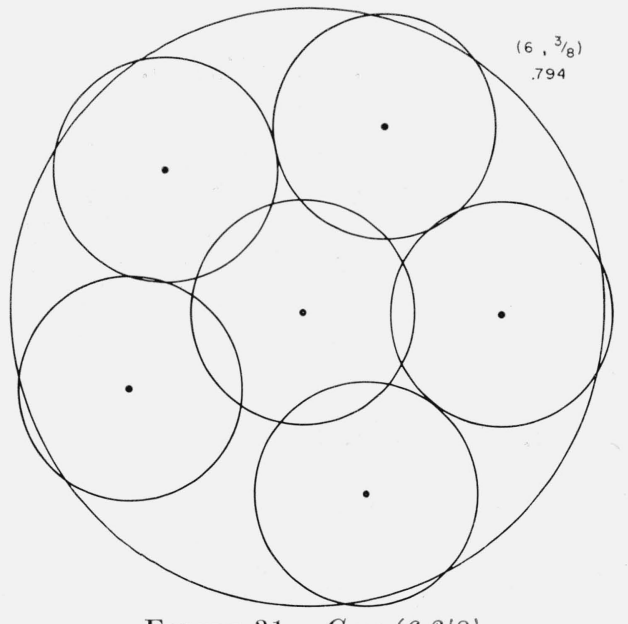

Figure 31 . Case $(6,3 / 8)$. 


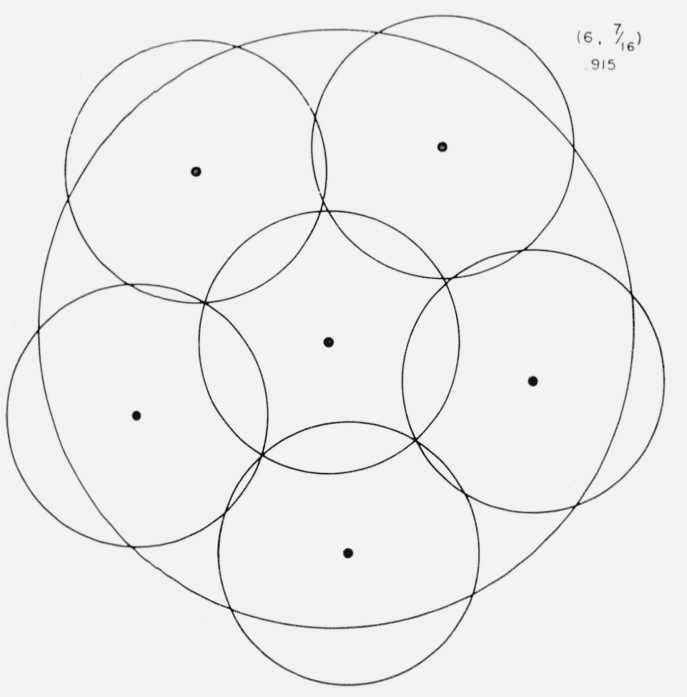

Figure 32. Case $(6,7 / 16)$.

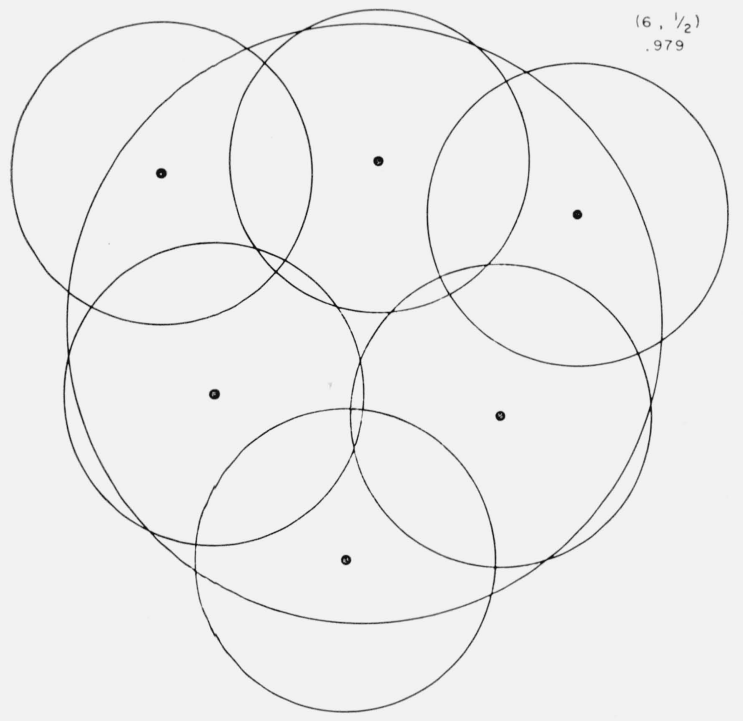

Figure 33. Case $(6,1 / 2)$.

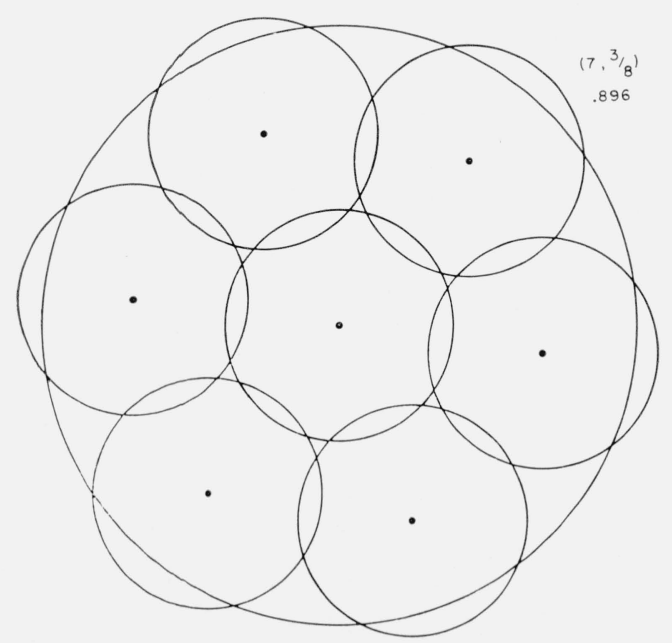

Figure 34. Case $(7,8 / 8)$.

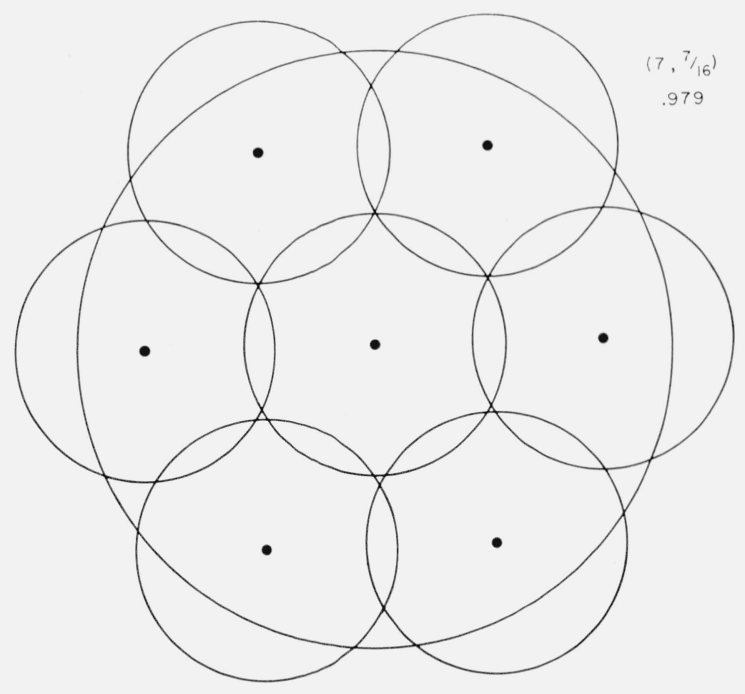

Figure 35. Case $(7,7 / 16)$.

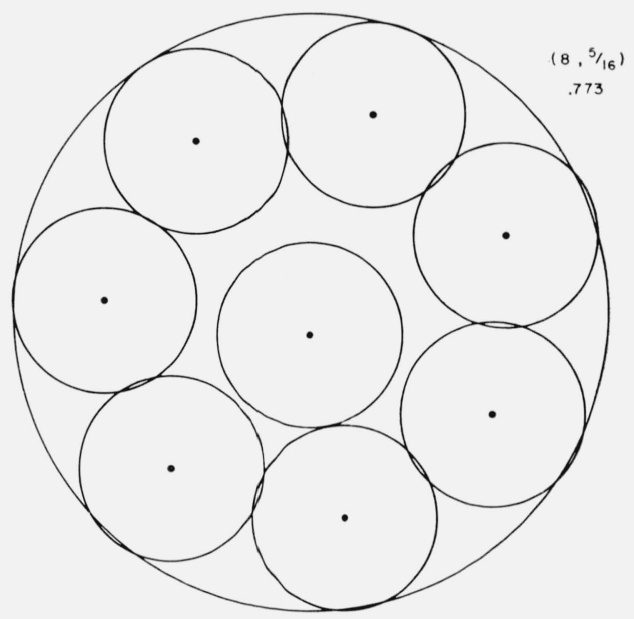

Figure 36. Case $(8,5 / 16)$.

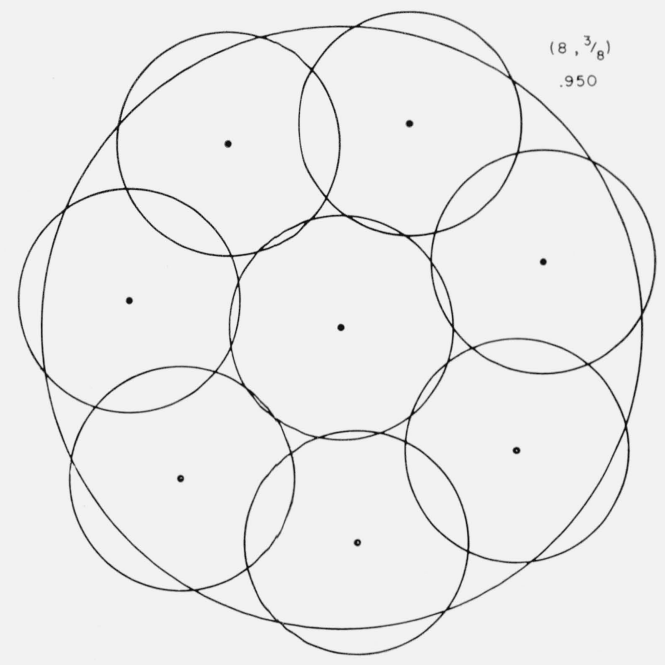

Figure 37. Case $(8,3 / 8)$. 


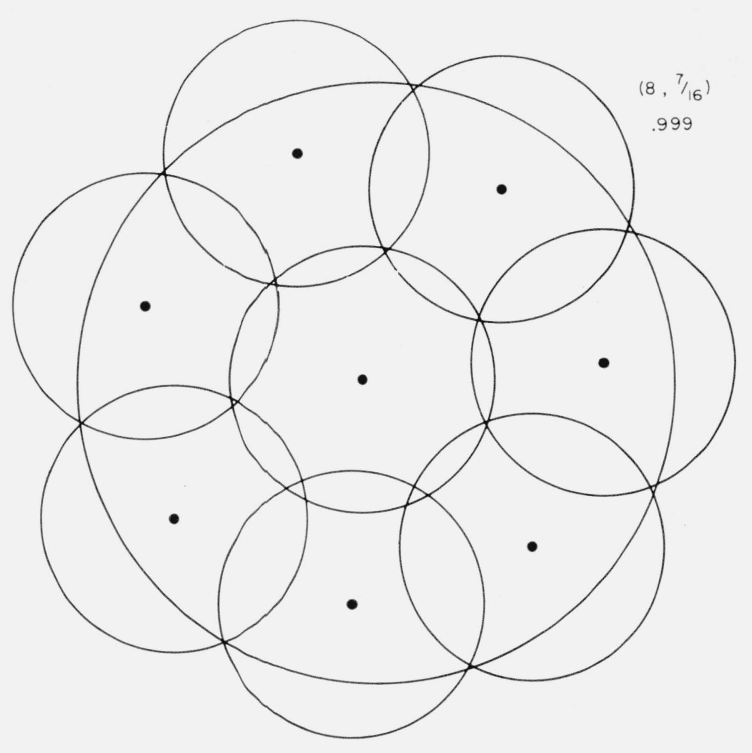

Figure $38 . \quad$ Case $(8, \% / 16)$.

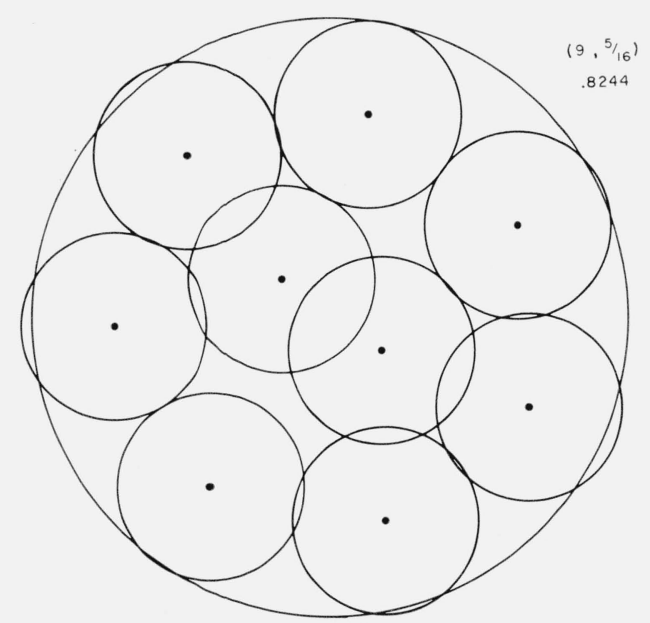

Figure 39. Case $(9,5 / 16) \quad$ Ratio $=0.8244$.

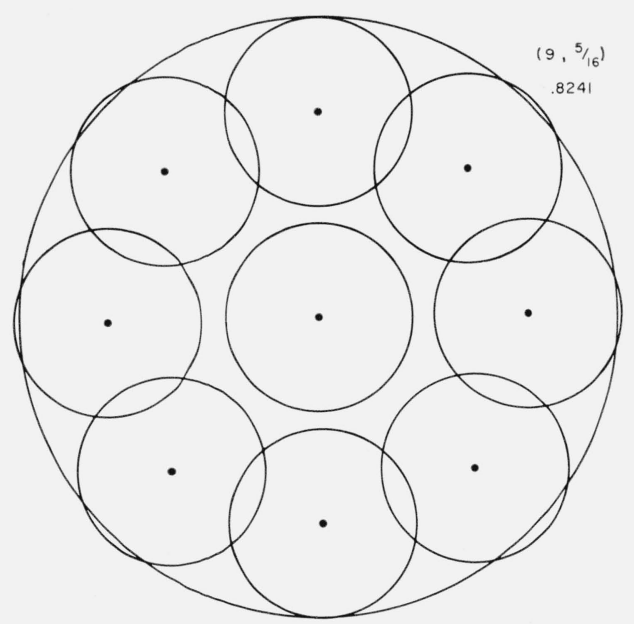

Figure 40. Case $(9,5 / 16) \quad$ Ratio $=0.8241$.

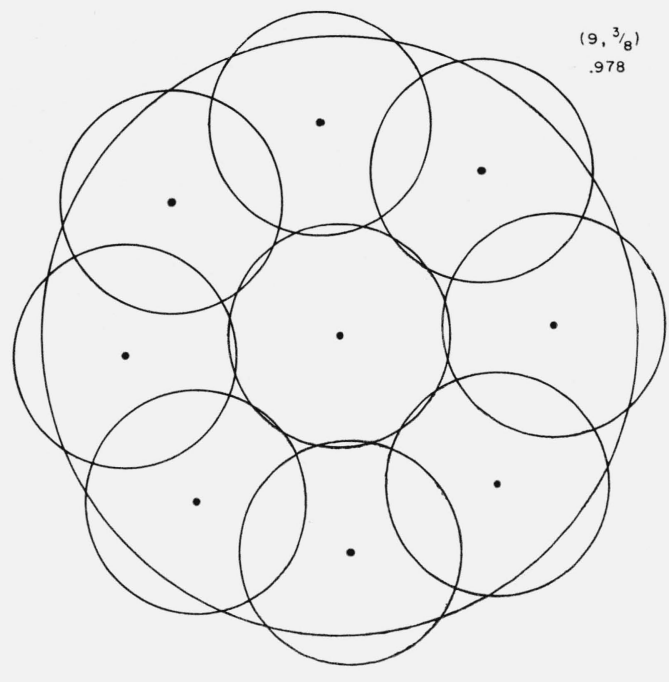

Figure 41. Case $(9,3 / 8)$.

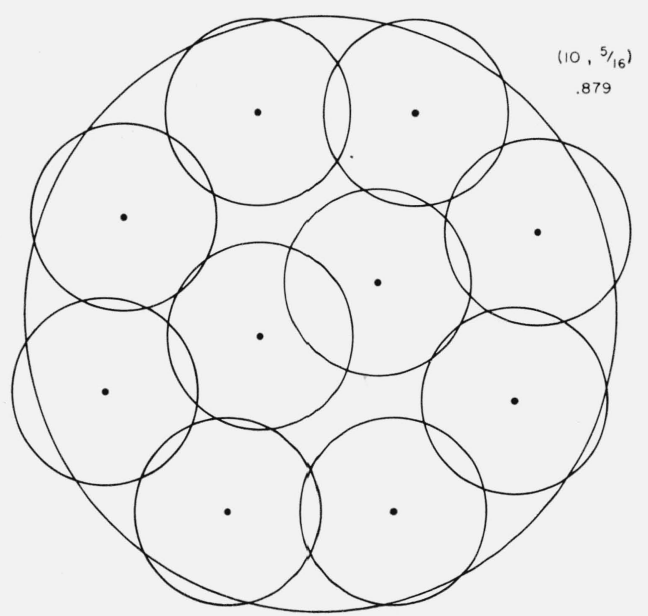

Figure 42. Case $(10,5 / 16)$.

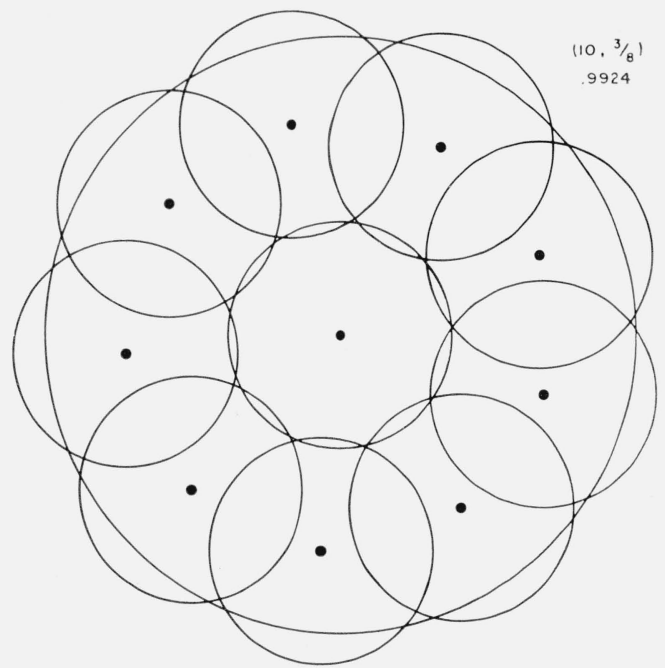

Figure 43. Case $(10,3 / 8) \quad$ Ratio $=0.9924$. 


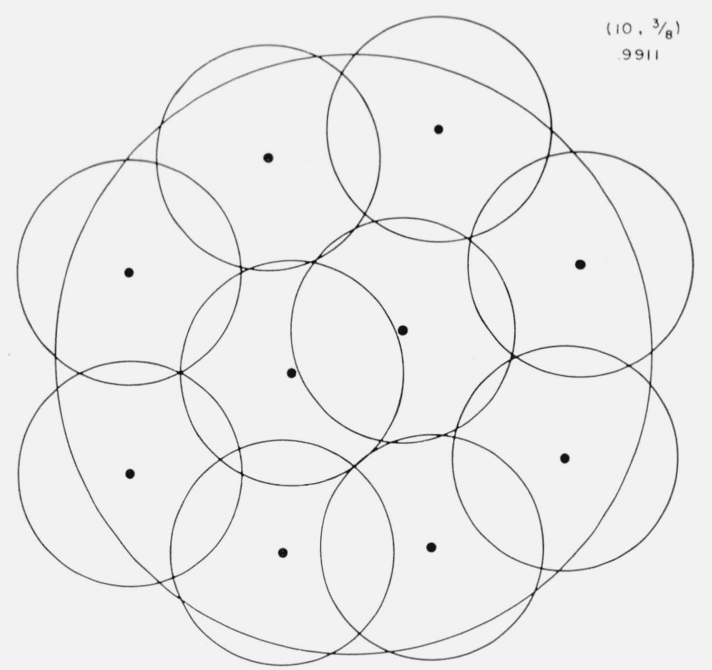

Figure 44. Case $(10,3 / 8) \quad$ Ratio $=0.9911$.

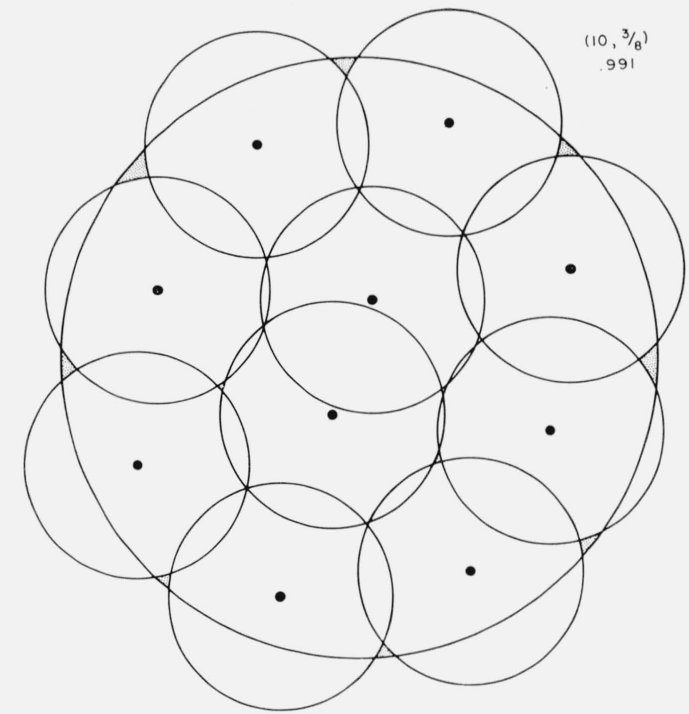

Figure 45. Case $(10,3 / 8) \quad$ Ratio $=0.991$.

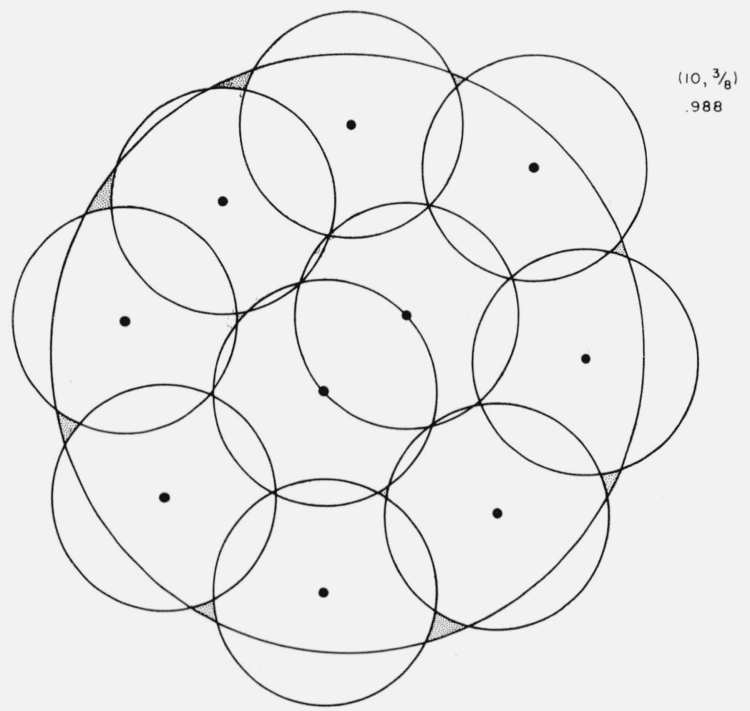

Figure 46. Case $(10,3 / 8) \quad$ Ratio $=0.988$.

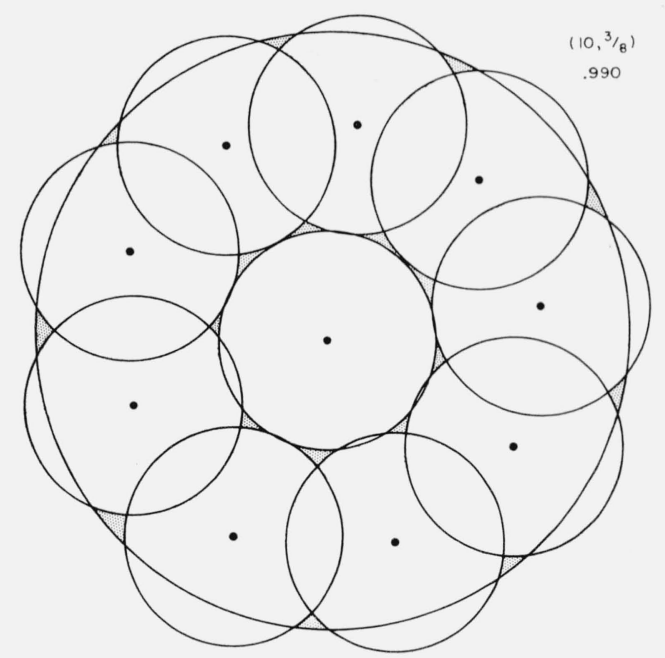

Figure 47. Case $(10,3 / 8) \quad$ Ratio $=0.990$.

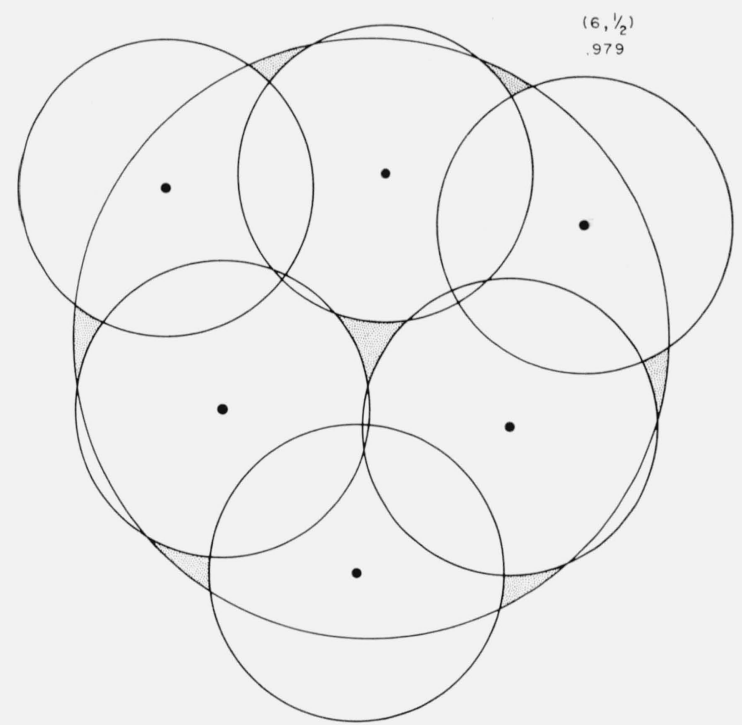

Figure 48. Case $(6,1 / 2)$.

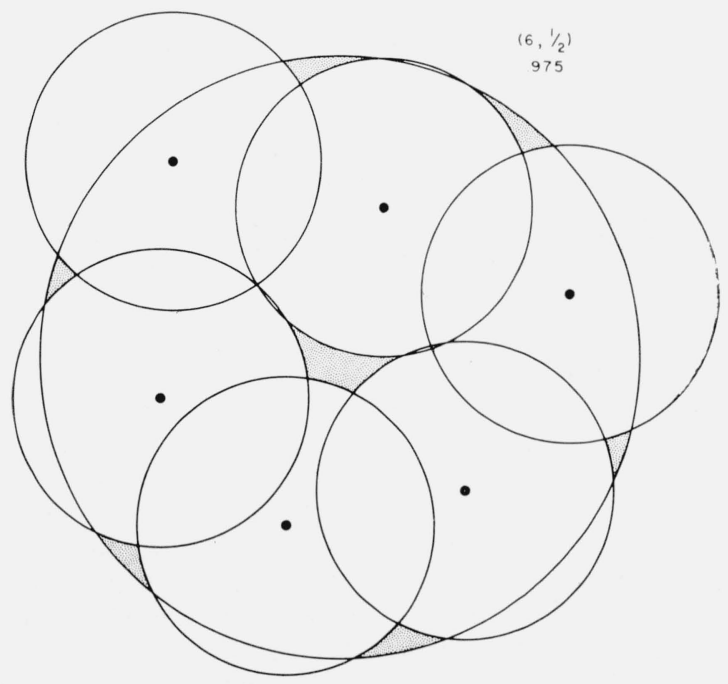

Figure 49. Case $(6,1 / 2)$. 


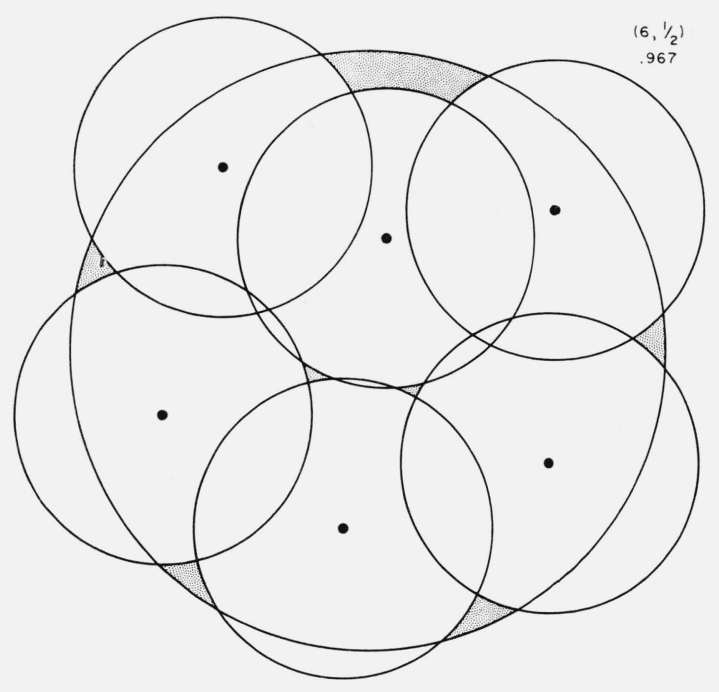

Figure 50. Case (6,1/2).

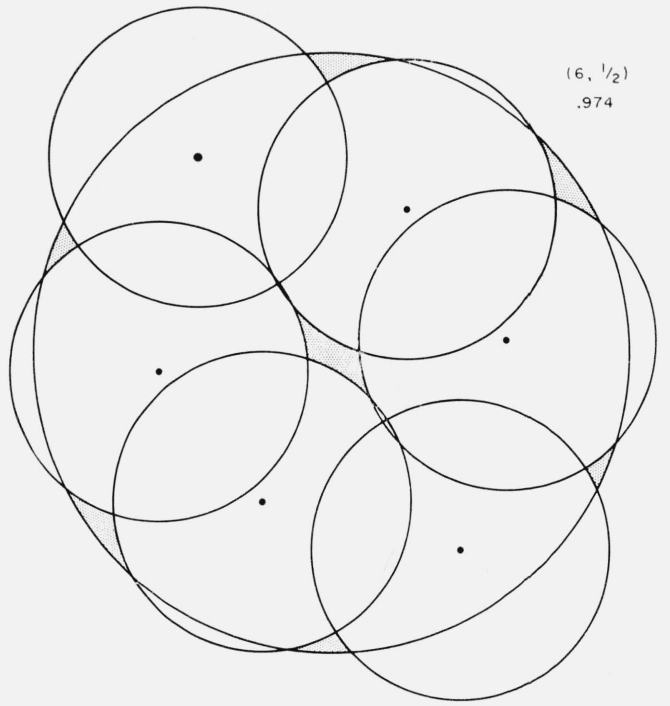

Figure 51. Case $(6,1 / 2)$.

all of the disks can be placed inside $A$ so as not to overlap each other; thus the ratio of coverage can be calculated accurately. The case $(6,9 / 16)$ is also "not interesting" because total coverage is possible. Figures 48 to 52 and 61 to 67 contain diagrams of the local maximum configurations for these cases.

An interesting phenomenon occurs in these cases. The best placement of the disks in case $(6,7 / 16)$ is a "central" or "flower-petal" configuration (see fig. 64), but in the case $\left(6, \frac{1}{2}\right)$ the same "central" configuration is merely the third best. This seems to indicate that there is an intermediate value of $r$ between $7 / 16$ and $1 / 2$, where the "central" and "triangular" (see fig. 48) configurations both cover equal portions of the total

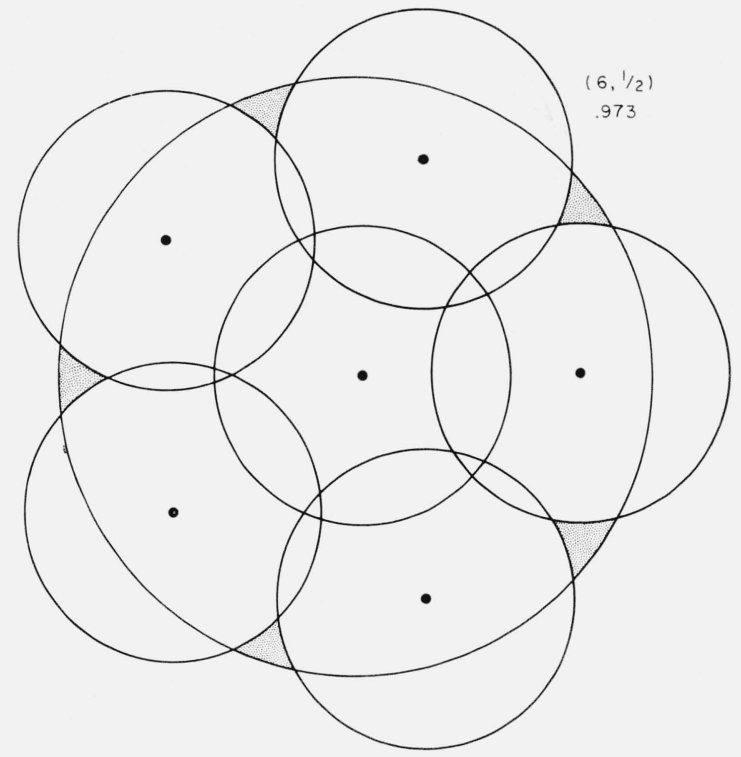

Figure 52. Case $(6,1 / 2)$.

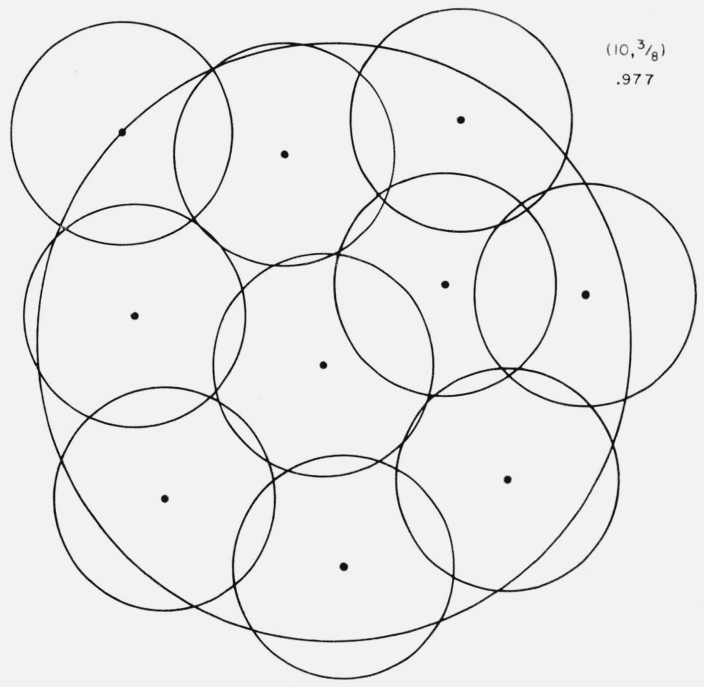

Figure 53. Case $(10,3 / 8)$.

area. The "ring" (see fig. 63 or 65) configuration of case $(6,7 / 16)$ seems to be a fairly natural analogy to either the "triangular" configuration or the "diamond" (see fig. 51) configuration of case (6, 1/2).

A further comparison was made between the "triangular" and "diamond" configurations. Three cases were used- $\left(6,{ }^{17 / 32}=0.531\right) ; \quad\left(6,{ }^{69} / 128=0.539\right)$; $\left(6,{ }^{35}{ }_{6}=0.547\right)$. In each case the "triangular" configuration of disks was the best, but its margin of victory decreased as the value of $r$ increased. Table 2 contains the results of the comparison. ${ }^{21}$

${ }_{21}$ The final mesh was 256 for all cases. 
Table 2. Comparison of two 6-disk configurations

\begin{tabular}{r|r|r|r}
\hline \hline \multicolumn{1}{r|}{} & Triangular & Diamond & Difference \\
\cline { 3 - 4 } 0.531 & 0.99591 & 0.99496 & 0.00095 \\
.539 & .99802 & .99768 & .00034 \\
.547 & .99932 & .99928 & .00004 \\
\hline
\end{tabular}

TaBle 3. Comparison of two 10-disk configurations

\begin{tabular}{r|r|r|r}
\hline \hline$r$ & \multicolumn{1}{r|}{ Central } & \multicolumn{1}{c|}{ Other } & \multicolumn{1}{c}{ Difference } \\
0.375 & 0.9924 & 0.9911 & 0.0013 \\
.383 & .9973 & .9957 & .0016 \\
.391 & .9996 & .9985 & .0011 \\
.398 & 1.0000 & .9998 & .0002 \\
.406 & 1.0000 & 1.0000 & .0000 \\
\hline
\end{tabular}

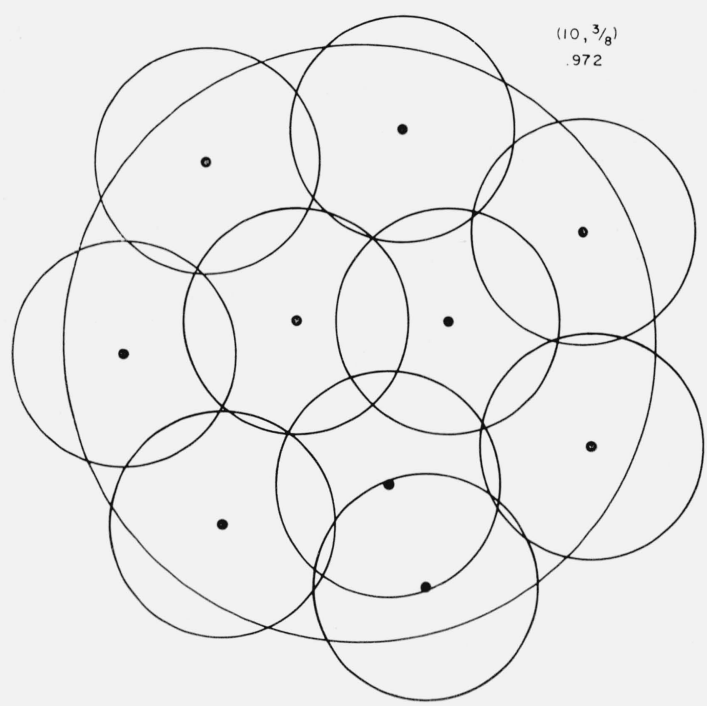

Figure 54. Case (10,3/8).

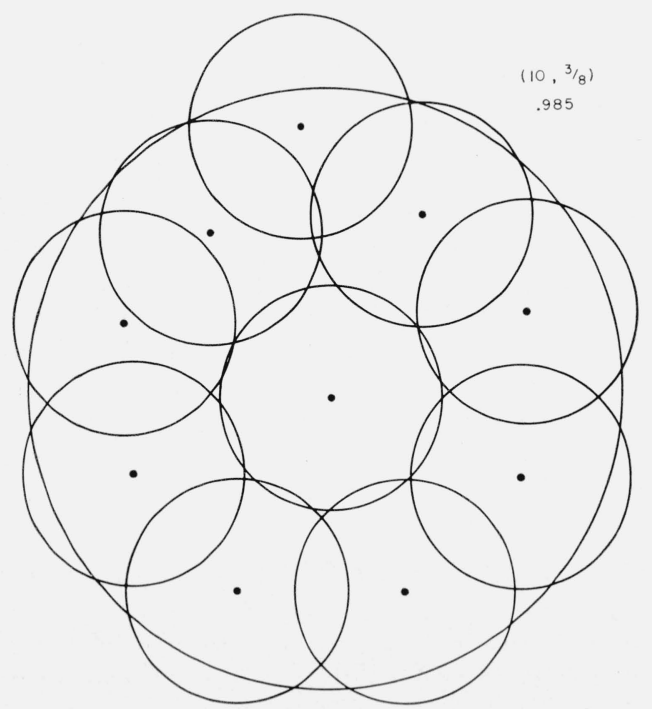

Figure 55. Case $(10,3 / 8)$.

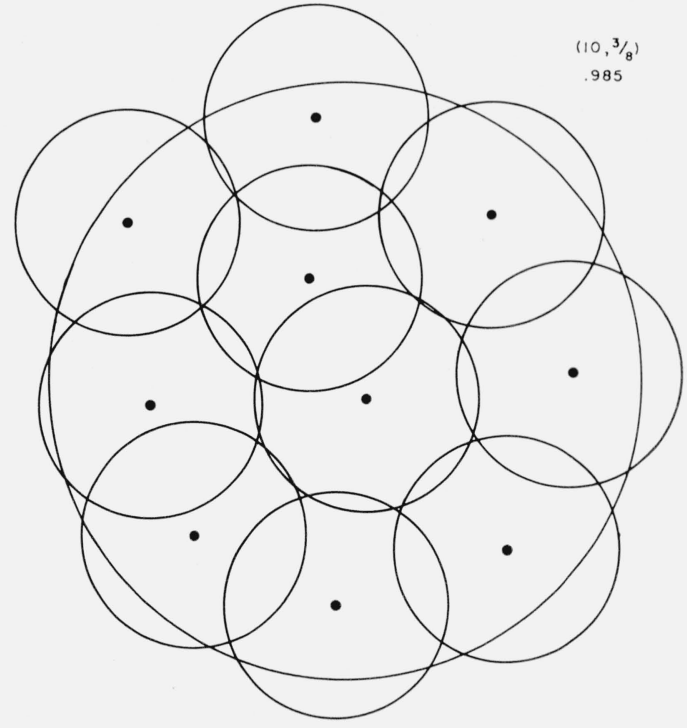

Figure 56. Case $(10,3 / 8)$.

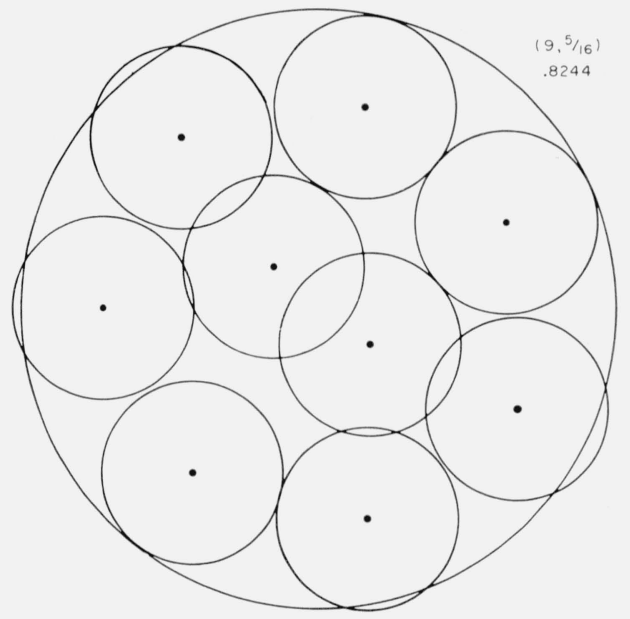

Figure 57. Case $(9,5 / 16)$.

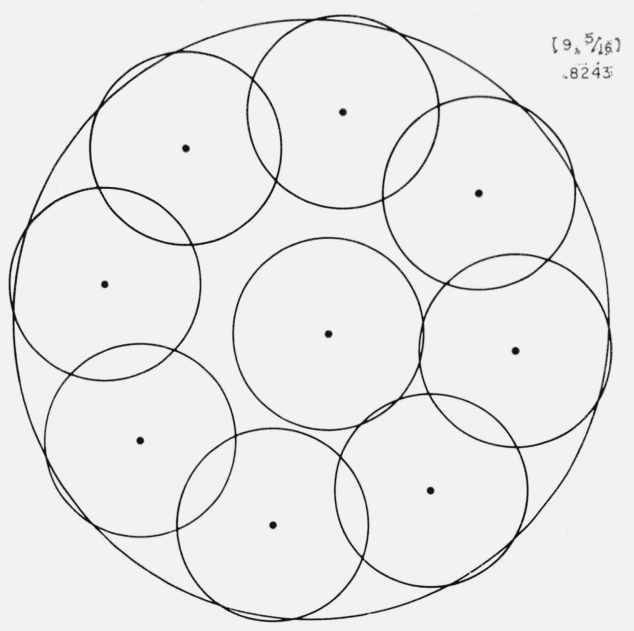

Figure 58. Case $(9,5 / 16)$. 


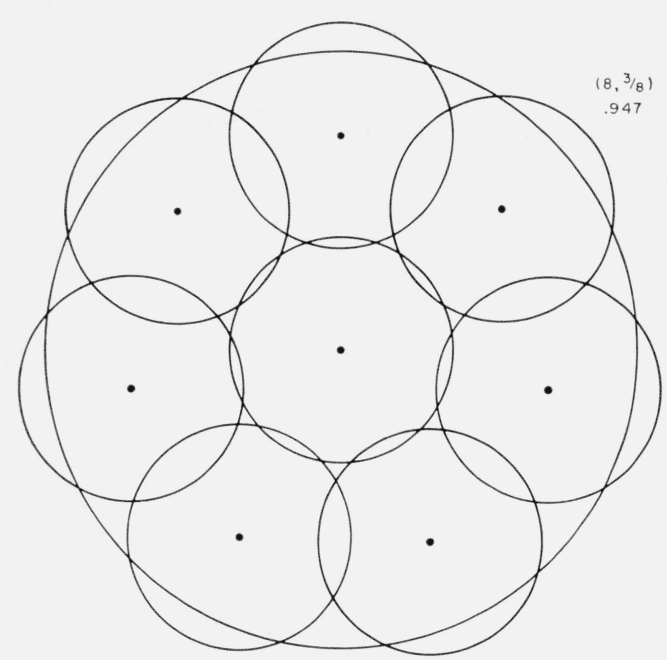

Figure 59. Case $(8,3 / 8)$.

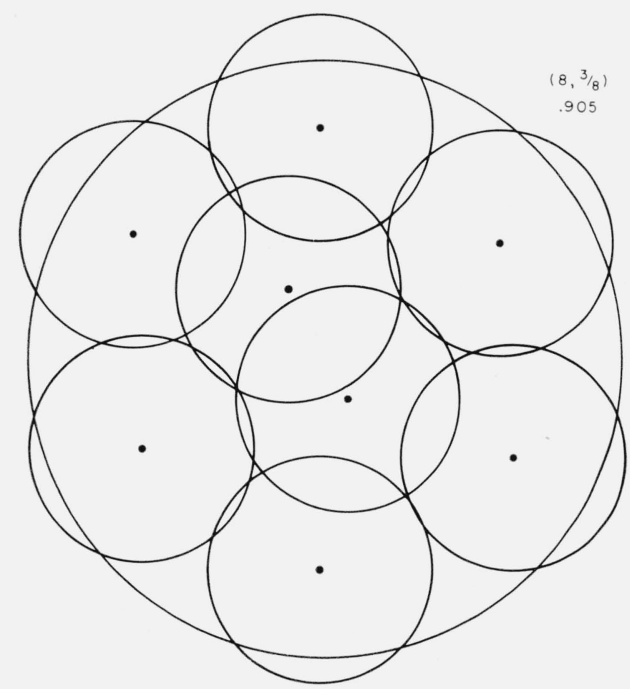

Figure 60. Case $(8,3 / 8)$.

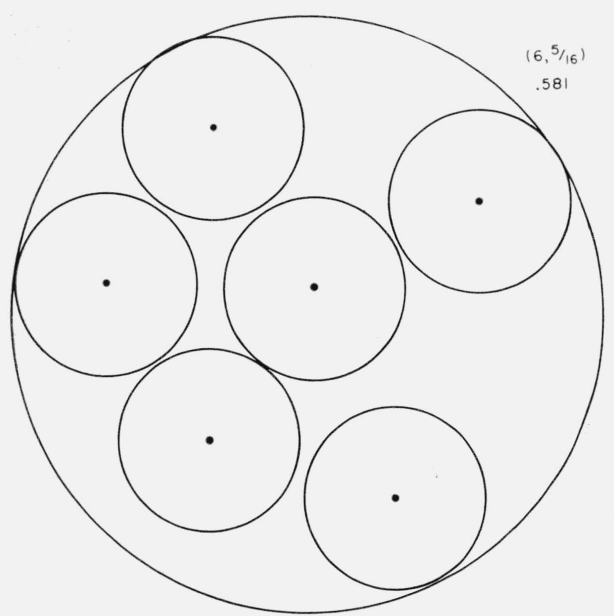

Figure 61. Case $(6,5 / 16)$.

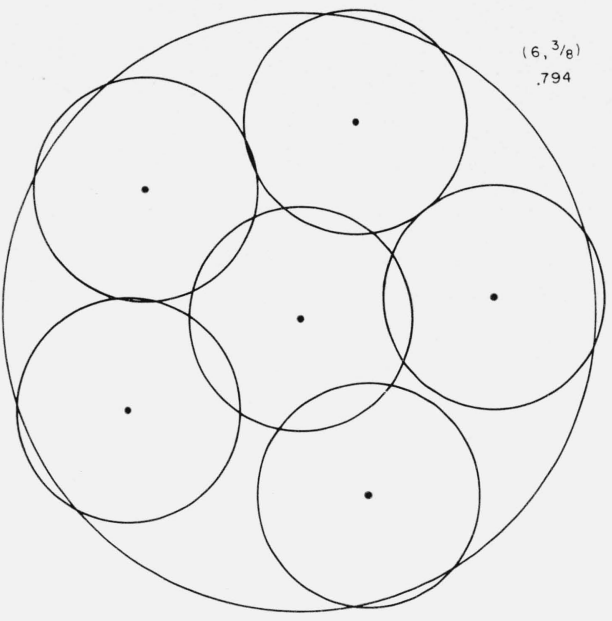

Figure 62. Case $(6,3 / 8)$.

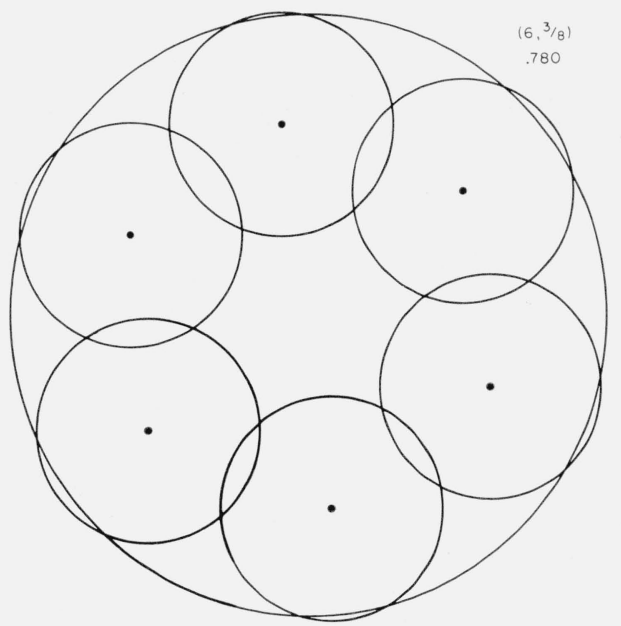

Figure 63 . Case $(6,3 / 8)$.

The chart shows that both configurations achieve total coverage at approximately equal values of $r$.

Runs were also made of several cases involving 10 disks at radii between $3 / 8=0.375$ and $13 / 32=0.406$. The two types of relative maxima were compared at five different values of the radius $r$. Diagrams of these relative maximum configurations for $r=3 / 8$ are contained in figures 45 and 47 . In all five cases the "central" configuration (fig. 47) was the best placement but usually by only a tenth of a percent. This data appears in table 3 .

Once again the two types of relative maxima achieve total coverage almost simultaneously.

\subsection{Comparison With Analytical Solution}

A run was made to compare our method with the analytical results of Neville for the case of five disks which was mentioned in section 2.3. According to Neville, the smallest value of $r$ for which coverage 


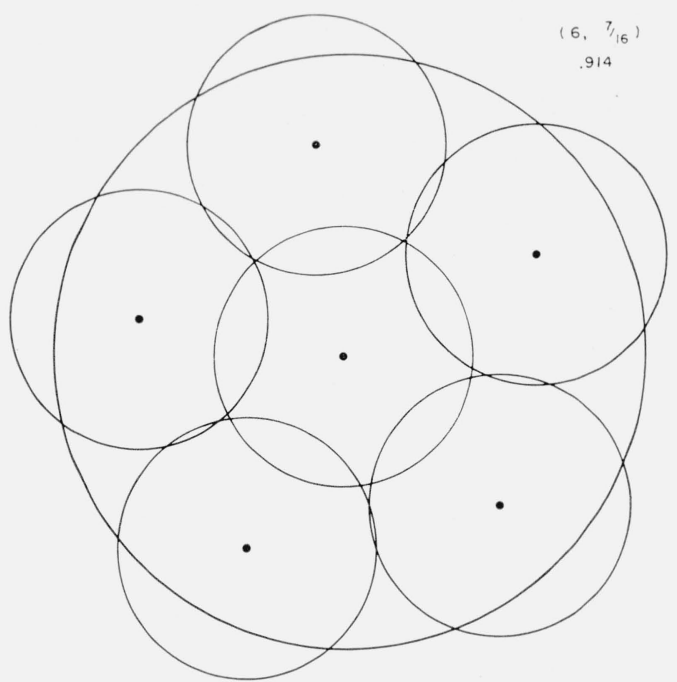

Figure 64 . Case $(6,7 / 16)$.

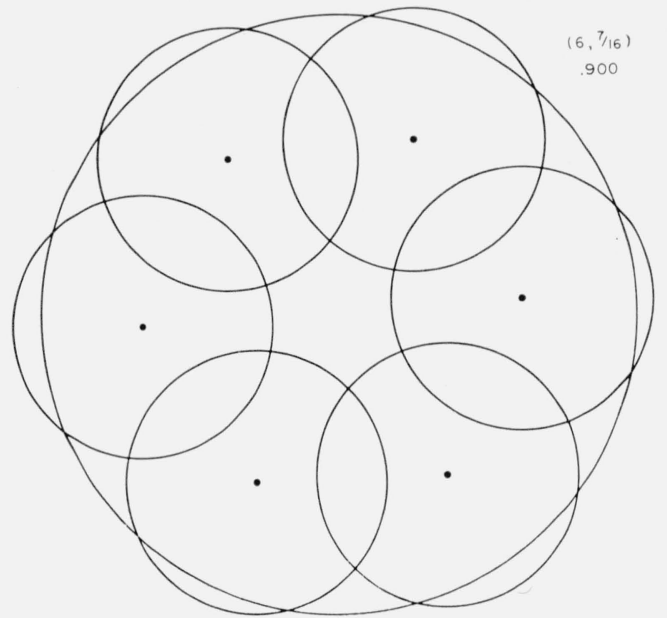

Figure 65. Case $(6,7 / 16)$.

with five disks is possible is 0.609375 ; this is called the "critical radius." The results of the run were quite surprising. The ratio of coverage was 0.999942 , just less than total coverage. It had not been suspected that the method we used would achieve a configuration exactly like that of Neville (see fig. 3), since other runs seemed to show that the ratio of coverage for configurations that are associated with almost total coverage is rather insensitive to changes in the configuration. This made it seem likely that the configuration achieved by the computer run would not resemble the Neville configuration too closely. The configuration arrived at by our searching technique (see fig. 68) bears a remarkable resemblance to that of Neville. Each configuration contains three disks that intersect in a point very near the center of $\mathrm{A}$ and two others symmetrically placed with centers substantially displaced from the center of $\mathrm{A}$.

An analytical solution to the covering problem for the cases where $n=2$ and $1 / 2<r<1$ is contained

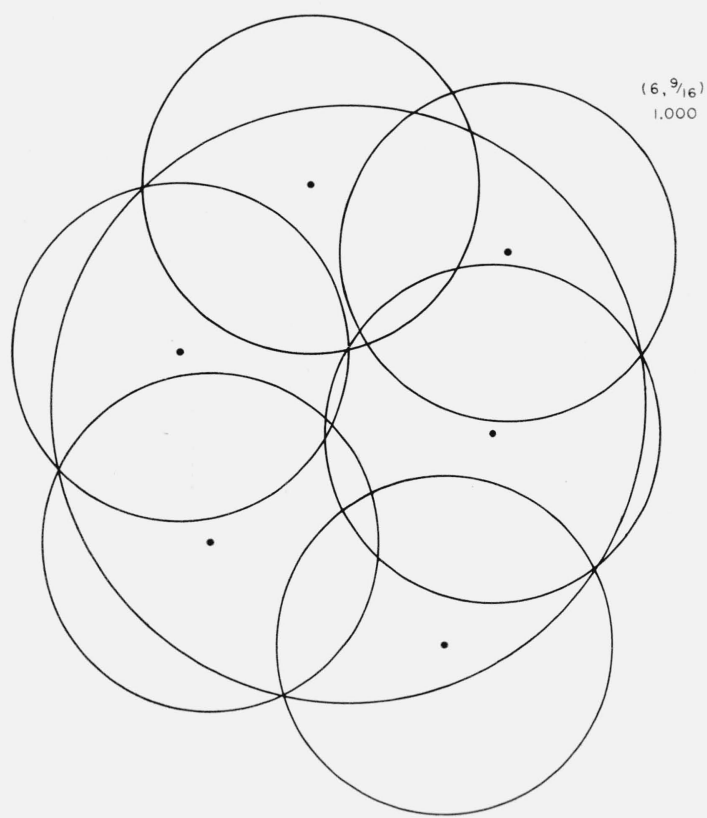

Figure 66 . Case $(6,9 / 16)$.

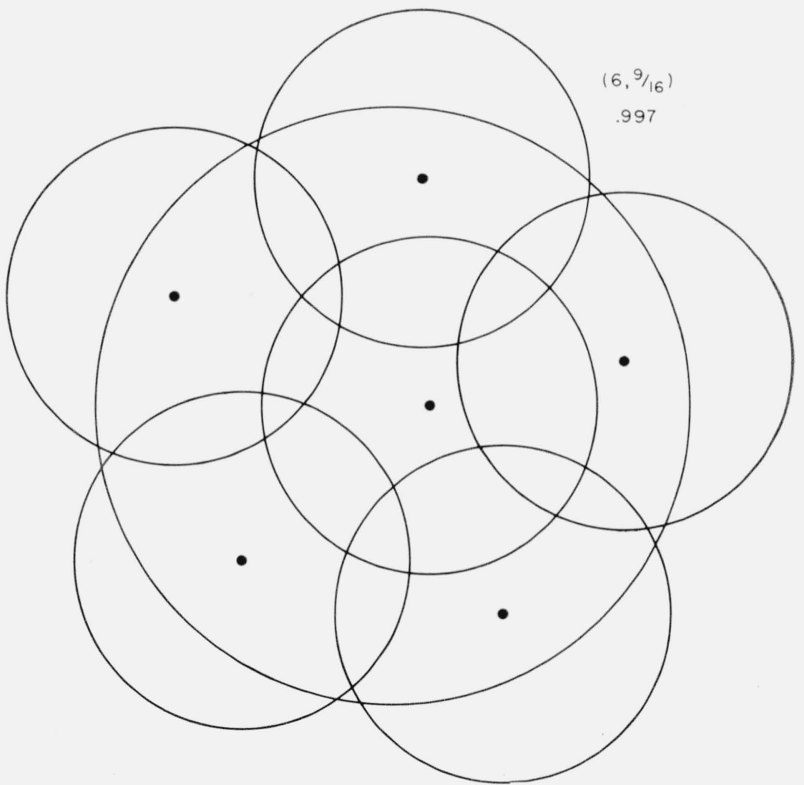

Figure 67. Case $(6,9 / 16)$.

Note: Figure 68 is located on p. 182.

in appendix 2. A comparison of the numbers gotten from computer runs with the true values showed excellent agreement, usually differing by less than 0.002 .

\subsection{Comparison of Search Methods}

A comparison was made of different methods of search to determine the trequency of occurrence of 


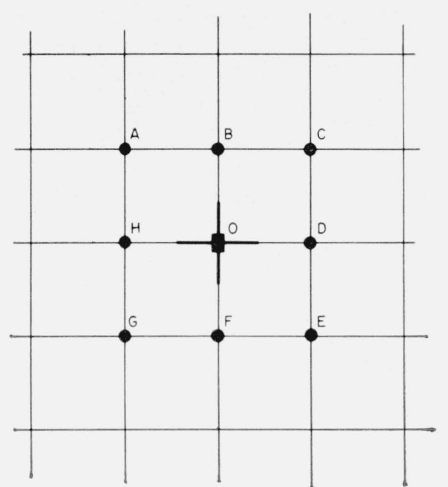

Figure 69. Neighborhood of a mesh point.

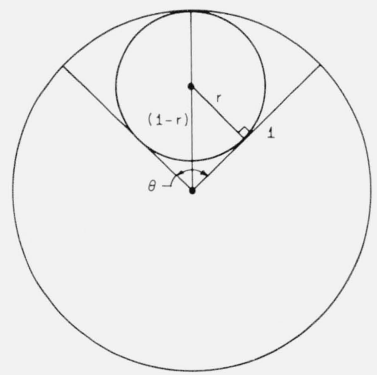

Figure 70. Maximum packing.

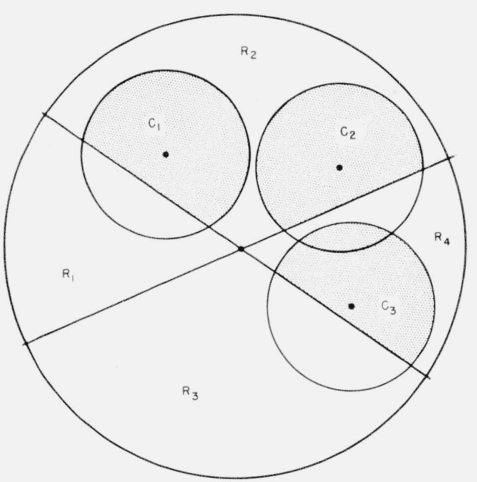

Figure 71. Wall interrupted coverage by three disks.

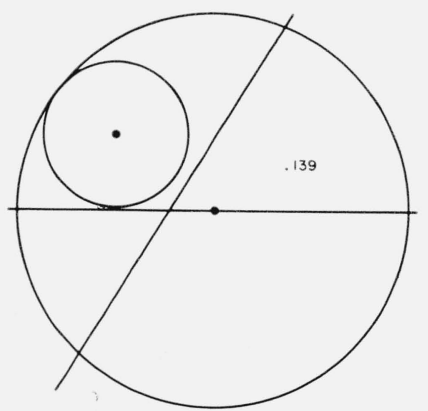

FiguRe 72. 1 disk coverage of wall region.

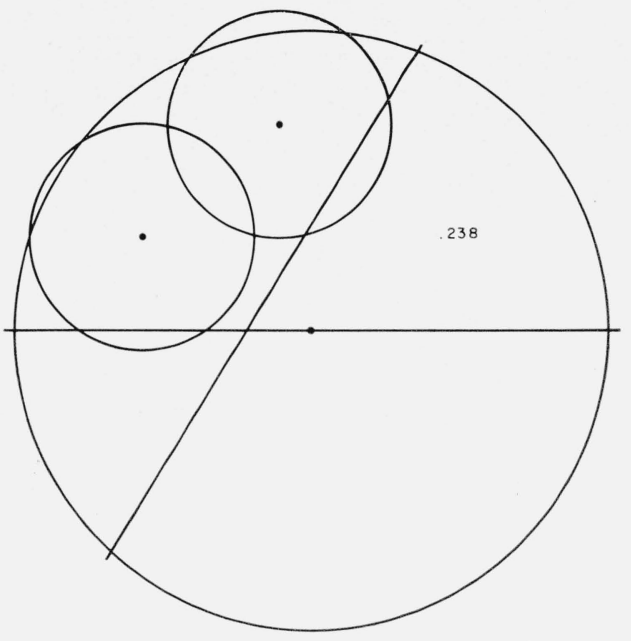

Figure 73. 2 disk coverage of wall region.

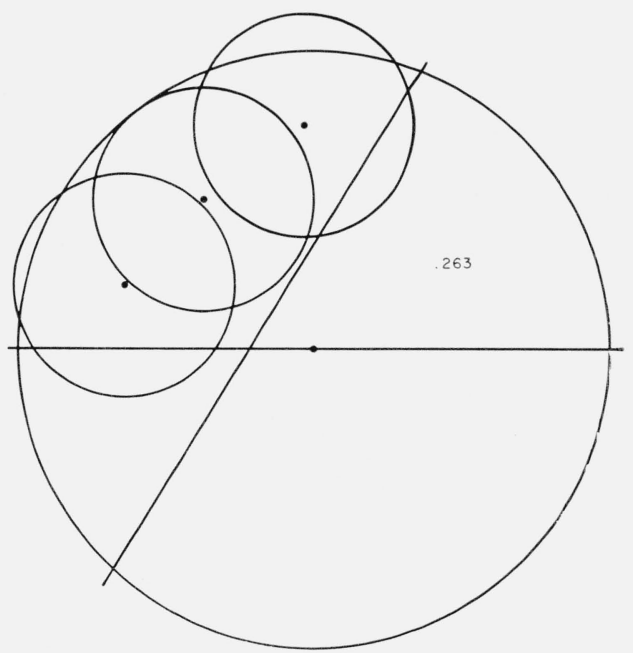

Figure 74. 3 disk coverage of wall region.

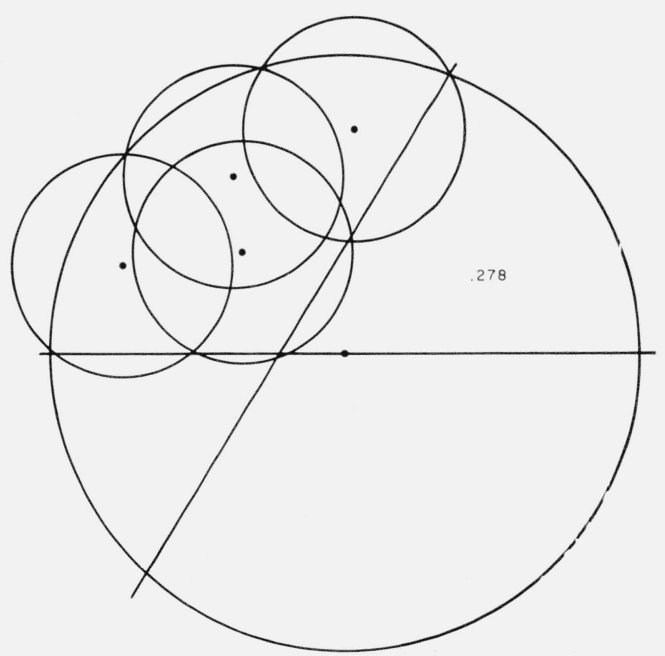

Figure $75 . \quad 4$ disk coverage of wall region. 
"global" maxima and to get some idea of the time required by the different methods. Five "methods" were programed and run. They are all derived from the three described in section 3.1, with minor variations.

(1) Plateau Steepest Ascent: This is basically the "steepest-ascent" method with two exceptions. First - if at some stage of the search there are no positive moves ${ }^{22}$ indicated for the current configuration $X$, then the neighborhood of $X$ is effectively enlarged by allowing each disk to move to any one of the eight grid-points surrounding its center (points $A$ through $H$ in fig. 69). This is done only when no moves are possible under the original definition of "neighbor." Second - if both of these searches fail to obtain a positive move then a search is made to determine if all four moves (that is, a move from 0 to one of B, D, F, H in fig. 69) of some disk are zero moves (i.e., leave RATIO unchanged). If such a disk is found, successive random placements of its center are tried until the value of RA'TIO is increased. If 25 trials fail to accomplish anything, the search is abandoned, and the refinement of mesh is made, etc.

This second feature is what gives the procedure the name "Plateau." In a function of a single variable $f(x)$ one says that a point $x_{0}$ is on a plateau of the function if all points in some neighborhood of $x_{\mathrm{o}}$ have equal tunction values. In two dimensions a plateau, analogously defined, can be visualized as being a flat portion of the surface represented by the function $g(x, y)$. An intuitive idea of the meaning of "plateau" is possible in these two cases precisely because a "picture" of the function can be represented in three dimensions or less.

Although we cannot visualize plateaus in higher dimensional spaces, we nevertheless define plateau analogously as a neighborhood of the space on which the function is defined throughout which the function is constant. In our case we have considered $F$ as a function of two variables, the coordinates of the center of a single disc, and so we have the type of plateau that makes sense visually.

It should be mentioned here that this particular feature of the "Plateau Steepest Ascent" method did not have nearly the same significance as did the first feature (the enlargement of neighborhood). That is to say, the results in using the steepest ascent method were more drastically altered by neighborhood enlargement than by the "plateau" feature.

(2) Least Positive Ascent: This is the "slowest ascent" described in section 3.1.

(3) First Positive Ascent: As described in section 3.1 .

(4) Cycle First Positive Ascent: This is the same as (3) except that the first disk whose moves are to be tried is the disk immediately following the disk that was last moved. The disks are ordered in a cycle $C_{1}, C_{2}, \ldots, C_{n-1}, C_{n}, C_{1}$, etc., for this purpose. This variation of (3) was used because the return to

${ }^{22}$ A positive move from a configuration $\mathrm{X}$ is effected by changing $\mathrm{X}$ to one of
its neighbors $\mathrm{X}_{1}$ such that $F\left(\mathrm{X}_{1}\right)>F(\mathrm{X})$. It is accomplished by a change in the its neighbors $\mathrm{X}_{1}$ such that $F\left(\mathrm{X}_{1}\right)>F(\mathrm{X})$. It is accomplished by a change in the
position of a single disk in one of the coordinate directions by one mesh unit.
$C_{1}$ at each stage seemed to introduce some bias that was undesirable.

(5) Cycle First Positive Ascent with Eight Degrees of Freedom: this resembles (4) except that throughout the search the neighborhood of $X$ is the enlarged neighborhood employed by the Plateau Steep Ascent.

Two cases, $(6,1 / 2)$ and $(10,3 / 8)$, were selected to be used in the comparison. Each case was known to have nonglobal local maxima that occurred quite frequently, and the configurations were quite distinct from a visual standpoint. Table 4 describes the frequency of occurrence ${ }^{23}$ of the global maximum in both cases, according to which method was used. The last column is probably the most significant because the cost depends on the time consumed and not the number of trials.

TABLE 4. Comparison of the 5 ascent methods for 2 cases

\begin{tabular}{|c|c|c|c|c|c|c|c|}
\hline Method & Case & Trials & $\begin{array}{c}\text { Global } \\
\text { max- } \\
\text { ima }\end{array}$ & Time & $\begin{array}{c}\text { Global } \\
\text { maxima } \\
\text { per trial }\end{array}$ & $\begin{array}{c}\text { Time } \\
\text { per } \\
\text { trial }\end{array}$ & $\begin{array}{c}\text { Global } \\
\text { maxima } \\
\text { per min }\end{array}$ \\
\hline th- & $\begin{array}{c}(6,1 / 2) \\
(6,1 / 2) \\
(6,1 / 2) \\
(6,1 / 2) \\
(6,1 / 2) \\
(10,3 / 8) \\
(10,3 / 8) \\
(10,3 / 8) \\
(10,3 / 8) \\
(10,3 / 8)\end{array}$ & $\begin{array}{l}25 \\
25 \\
25 \\
25 \\
25 \\
25 \\
21 \\
24 \\
25 \\
20\end{array}$ & $\begin{array}{r}13 \\
10 \\
11 \\
7 \\
8 \\
5 \\
0 \\
0 \\
5 \\
5\end{array}$ & $\begin{array}{r}\text { Min. } \\
44 \\
47 \\
34 \\
19 \\
52 \\
82 \\
89 \\
71 \\
40 \\
90\end{array}$ & $\begin{array}{r}0.52 \\
.40 \\
.44 \\
.28 \\
.32 \\
.20 \\
.00 \\
.00 \\
.20 \\
.25\end{array}$ & $\begin{array}{r}\text { Min. } \\
\text { 1. } 76 \\
1.88 \\
1.36 \\
.76 \\
2.08 \\
3.28 \\
4.24 \\
2.96 \\
1.60 \\
4.50\end{array}$ & $\begin{array}{r}0.295 \\
.213 \\
.324 \\
.368 \\
.154 \\
.061 \\
.000 \\
.000 \\
.125 \\
.056\end{array}$ \\
\hline
\end{tabular}

From this point of view, the Cycle First Positive Ascent (4) is clearly the best. There is another point of view, however, that may be still more significant in a comparison of these five methods. The point is this - the method to be preferred is the method that tends to reach more different peaks. Such a method would reach the global maximum less often but one would feel more confident of the highest peak among 5 or 10 than he would if only 2 or 3 distinct peaks had been found. Table 5 contains a record of the number of distinct peaks that were found by each method. It seems to indicate that the variations of the "first positive ascent" method are better than the others at locating different peaks.

TABLE 5. Number of different peaks found by the 5 methods

\begin{tabular}{|c|c|c|c|c|c|}
\hline \multicolumn{6}{|c|}{ Case $(6,1 / 2)$} \\
\hline $\begin{array}{l}\text { Method } \\
\text { Peaks. }\end{array}$ & $\begin{array}{l}1 \\
3\end{array}$ & $\begin{array}{l}2 \\
4\end{array}$ & $\begin{array}{l}3 \\
4\end{array}$ & $\begin{array}{l}4 \\
3\end{array}$ & $\begin{array}{l}5 \\
4\end{array}$ \\
\hline $\begin{array}{l}\text { Method } \\
\text { Peaks. }\end{array}$ & $\begin{array}{l}1 \\
3\end{array}$ & $\begin{array}{l}2 \\
3\end{array}$ & $\begin{array}{l}3 \\
4\end{array}$ & $\begin{array}{l}4 \\
5\end{array}$ & $\begin{array}{l}5 \\
3\end{array}$ \\
\hline
\end{tabular}

\subsection{Critical Values of Radius}

Considering problems (c) and (d) of section 1.2 once again, we define the "low critical radius" of $n$ disks $r_{1}(n)$ as the answer to problem (d) and the

23 Two final configurations are deemed equivalent, in this comparison, if they are similar geometrically and achieve nearly identical values of RATIO. 
"high critical radius," $r_{2}(n)$ as the answer to problem (c). Neville [1] refers to the latter simply as the "critical radius" but we want to study both. For any number of disks $n$ the cases that are "interesting" are the cases $(n, r)$ where $r_{1}(n) \leq r \leq r_{2}(n)$.

A determination was made of $r_{1}(n)$ and $r_{2}(n)$ for all $n$ in the range $1 \leq n \leq 10$ (table 6 ). Some of the values were found by using the search methods and others could be determined analytically. For example we can calculate $r_{1}(n)$ for $2 \leq n \leq 5$ by the following argument:

We want to place the $n$ disks in a ring around the center of $A$ in such a way that the disks are packed in as tightly as possible without overlapping. Under these conditions each disk requires a sector cut off by an angle $\theta=2 \pi / n$. Referring to the diagram in figure 70 we can immediately write

$$
r /(1-r)=\sin \theta / 2 \quad \text { and } \theta=2 \pi / n,
$$

which reduces finally to

$$
r=\frac{\sin (\pi / n)}{1+\sin (\pi / n)}
$$

\begin{tabular}{|c|c|c|c|c|c|c|c|c|c|c|}
\hline$n_{-.}$ & 1 & 2 & 3 & 4 & 5 & 6 & 7 & 8 & 9 & 10 \\
\hline $\begin{array}{l}(n) \\
(n)\end{array}$ & $\begin{array}{l}1.0 \\
1.0\end{array}$ & $\begin{array}{r}0.50 \\
1.0\end{array}$ & $\begin{array}{r}0.464 \\
.866\end{array}$ & $\begin{array}{r}0.414 \\
.707 \\
.903\end{array}$ & $\begin{array}{r}0.370 \\
.609 \\
.30\end{array}$ & $\begin{array}{r}0.333 \\
.555 \\
202\end{array}$ & $\begin{array}{r}0.333 \\
.500 \\
167\end{array}$ & $\begin{array}{r}0.302 \\
.437 \\
135\end{array}$ & $\begin{array}{r}0.276 \\
.422\end{array}$ & $\begin{array}{r}0.266 \\
.398 \\
.39\end{array}$ \\
\hline$r_{\ldots} \ldots$ & 0 & .500 & .402 & .293 & .239 & .222 & .167 & .135 & .146 & \\
\hline
\end{tabular}

TABLE 6. Low and high critical radii for up to 10 disks

and this formula is valid so long as the ring configuration is clearly the optimum packing.

For small values of $n, r_{2}(n)$ can be calculated by noting that to achieve total coverage, all of the circumference of $A$ must be covered. For a given $n$ the minimum $r$ required to cover the circumference is given by:

$$
r=\sin \pi / n .
$$

If total coverage is in fact achieved at this value of $r$ then $r_{2}(n)$ has been found. This argument was found to be valid for $n=2,3,4$.

Table 6 shows that the length $\Delta r$ of the interval $\left(r_{1}(n), r_{2}(n)\right)$ shows a tendency to decrease with $n$, although not monotonically. Thus for larger $n$ there is a smaller range of interesting cases. In fact when $n=10$ the interval is $(0.266,0.398)$ whose length is only 0.132 , whereas when $n=2$ the interval is $(0.50,1.0)$ of length 0.500 .

\subsection{Efficiency of Covering Configuration}

It was thought that some measure of the efficiency of a covering might be useful in some applications. The efficiency should give some indication of ratio of coverage versus total covering area available. We therefore define the efficiency $E(n, r)$ as the ratio between the maximum area (not percent!) coverable by $n$ disks of radius $r$, and the total composite area of the $n$ disks. If we denote the best percent coverage by $C(n, r)$ and remember that we have been assuming $R=1$ we obtain the formula for efficiency:

$$
E(n, r)=\frac{\pi C(n, r)}{n \pi r^{2}}=\frac{C(n, r)}{n r^{2}}
$$

The values of $C(n, r)$ are contained in table 1 , and $E(n, r)$ in table 7 . For most pairs $(n, r)$ the efficiency is greater than $1 / 2$ but two cases were found where it was less than $1 / 2$. Specifically, $E(3,7 / 8)=0.435$ and $E(4,3 / 4)=0.444$.

TABLE 7. Efficiency of coverage, $\mathrm{E}(\mathrm{n}, \mathrm{r})$

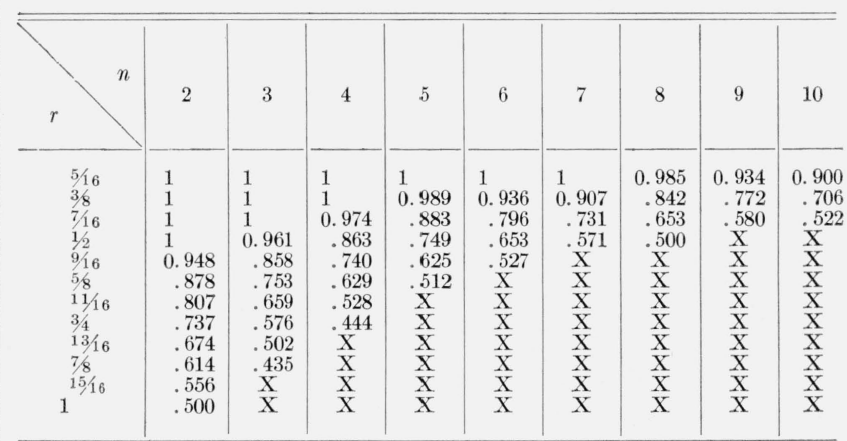

$\mathrm{X}$ indicates total coverage is possible, so increases in $P=n r^{2}$ are just wasted.

TABLE 8. Comparison of covering efficiency with $\mathrm{nr}^{2}$ held constant

\begin{tabular}{l|l|l|l|l|l|l|l}
\hline & & & & & & \\
Case & $(4,39 / 64)$ & $(5,35 / 64)$ & $(6,1 / 2)$ & $(7,15 / 32)$ & $(8,7 / 16)$ & $(9,13 / 32)$ & $(10,25 / 64)$ \\
Ratio & 0.973409 & 0.980666 & 0.979418 & 0.995094 & 0.999432 & 0.999130 & 0.999626 \\
$n r^{2}$ & 1.485 & 1.495 & 1.500 & 1.538 & 1.531 & 1.485 & 1.526 \\
$E(n, r)$ & 0.655 & 0.656 & 0.653 & 0.647 & 0.653 & 0.673 & 0.655 \\
\hline
\end{tabular}

Several runs were made to obtain data pertaining to problem (e) of section 2.2. The value of $P=n r^{2}$ was set at 1.5 (or as nearly as possible) ${ }^{24}$ and the corresponding efficiencies were calculated. At first it was suspected that efficiency would always be better when $n$ was larger, but the results in table 8 contradict the conjecture. The efficiency of $(5,35 / 64)$ is 0.656 and that of $(6,1 / 2)$ is only 0.653 . This discrepancy could hardly be due to the errors involved in the approximation because the approximation tends almost always to be an under estimate of the true value (see sec. 6).

The Kerschner result cited in section 1.3 can be rewritten slightly to read:

$$
\lim _{\substack{r \rightarrow 0 \\ n \rightarrow \infty}} \frac{A}{N(r) \pi r^{2}}=9 / 2 \pi \sqrt{3}=0.827 .
$$

The expression on the left is the limit of the efficiency in the case of total coverage as the number of circles increases and $r$ decreases.

Denoting $\lambda=2 \sqrt{3} / 9$ we get the following corollary to the Kerschner theorem:

24 The possible values of $r$ are restricted to numbers of the form $k / 64$ where $k$ is a positive integer $<64$. 
Given a set $S$ of area $P / \lambda$ and $\epsilon>0$, there exists a number $r_{0}>0$ such that $\left|r^{2} N(r)-P\right|<\epsilon$ when $r<r_{0}$.

This follows immediately by writing Kerschner's theorem as:

$$
\lim _{r \rightarrow 0} r^{2} N(r)=\lambda(P / \lambda)=P
$$

If we define the potential $P$ of a set of $N$ disks of radius $r$ as $P=N r^{2}$, the above corollary says that any set of area $P / \lambda$ can be covered by a set of disks with potential arbitrarily close to $P$.

In particular, if the set is a disk of radius $R$, then we have $\pi R^{2}=P / \lambda$ or $R=(P / \pi \lambda)^{1 / 2}$ where $1 / \pi \lambda=0.827$. If we put $P=1.5$ we get $R=1.114$ and this means that a disk of radius 1.114 can be "almost" covered by disks with a potential of 1.5 .

\section{Convergence of the Approximation}

Some estimates of the accuracy of the approximation used in the search methods have been determined. The approximation was introduced in the calculation of the ratio of coverage (RATIO). The convergence of the approximation as the mesh is refined was studied by an auxiliary computer program called RATIO CONVERGENCE which calculates the value of RATIO for a particular configuration at mesh sizes $8,16,32,64,128$. Several of the configurations were simple enough so that the ratio of coverage could also be calculated accurately by analytical methods. Table 9 gives the results of several runs performed to estimate the convergence.

The configuration of the first case in table 9 consists of a single disk of radius $r=1 / 2$ centered at the center of $A$. This means that the ratio of coverage should be exactly 0.250 . The table indicates that at a mesh of 64 the approximation differs by only 0.0006 from the correct ratio of coverage..$^{25}$ At a mesh of 256 the difference is only 0.00016 .

In the first three cases the change in RATIO between mesh 64 and mesh 128 was less than or equal to 0.0004 .

In the fourth case the configuration involved two disks and the correct ratio of coverage was computed using the algorithm referred to in section 2.2 . The true ratio of coverage was found to be 0.40225 , which means the approximation differs by less than 0.001 from the correct value at mesh 64 .

Two other configurations were tried in which all disks lie entirely inside $A$ and no overlap occurs. The cases were $(6,5 / 16)$ and $(6,2 / 8)$. In the former case the approximate ratio at mesh 256 was 0.584940 and the true ratio was 0.585937 , a difference of 0.001 . In the latter case the approximate ratio was 0.374503 and the exact value 0.375000 , a difference of 0.0005 .

These tests of the accuracy of the approximation seem to indicate that at a mesh of 64 one can expect

${ }^{25} \mathrm{~A}$ final mesh of 64 was the one used in most of the computer maximization experiments.
TABLE 9. Comparison of ratio convergence at different meshes

\begin{tabular}{l|r|r|r|r|r}
\hline \hline & & & & \\
\\
Mesh
\end{tabular}

accuracy $^{26}$ on the order of \pm 0.002 . Furthermore, the approximation was usually an underestimate of the true value. At a mesh of 256 the accuracy is usually about twice as good. Most runs used a final mesh of 64 since the additional time required to increase to a mesh of 256 did not seem to be warranted by the additional accuracy obtained.

An attempt was made to obtain rigorous limits for the error involved in approximating the covered area by the number of grid-points.

Let $L(r)$, denote the number of lattice points inside the disk defined by $x^{2}+y^{2} \leq r^{2}$, and let

$$
D(r)=\left|\pi r^{2}-L(r)\right| \text {. }
$$

It is easily shown [18] that $D(r)$ converges to zero as $r \rightarrow \infty$, but the question of just how fast is another matter entirely.

Hilbert and Cohen-Vossen show that

$$
D(r) \leq 4 \sqrt{2} \pi r
$$

or in other words

$$
D(r)=0(r) .
$$

Landau [19, pp. 183-278] shows that

$$
D(r)=0\left(r^{2 / 3}\right),
$$

in fact $[19$, p. 271],

$$
D(r)=0\left(r^{37 / 56+\epsilon}\right) \text { for any } \epsilon>0,
$$

but that

$$
D(r) \neq 0\left(r^{1 / 2}\right) .
$$

These have been somewhat improved [20] to read,

$$
D(r)=0\left(r^{15 / 23+\epsilon}\right) \text { for any } \epsilon>0 .
$$

The best result to date seems to be

$$
D(r)=0\left(r^{13 / 20}\right)
$$

which was shown by Loo-Keng Hua in 1940 [21].

The "conjectured" result is

$$
D(r)=0\left(r^{1 / 2+\epsilon}\right) \text { for } \epsilon>0
$$

26 This refers to the error in estimating $F(X)$ by the approximation $n_{2} / n_{1}$ defined in section 32 . The error involved in estimating a relative maximum of $F(X)$ by one of the final configurations produced by the computer program is somewhat one of ther.
larger. 
and it has already been pointed out above that this is the best result possible.

Obtaining numerical upper bounds for $D(r)$ from these results would involve a great deal more effort than seems worthwhile here. The problem is clearly tied in with fairly abstruse number theoretical investigations. Furthermore, the error bounds that may be derived from the Hilbert and Cohen-Vossen inequality are so bad that there is some real doubt as to whether the subsequent tightening of this inequality is substantial enough to help us out.

There is also the problem of estimating the error for $n$ possibly (and indeed probably) overlapping disks. The above results pertain to the estimation of the area of a single disk without any bites taken out of it, whereas we have a considerably more involved situation, especially since we really want as tight an inequality as possible.

A recent paper by H. L. Mitchell III [22] gives a large amount of numerical results concerning $L(r)$, the number of lattice-points (grid-points) in a circle of radius $r$. The paper includes calculations of $D(r)$ and also $D(r) / r^{1 / 2}$. The latter values were calculated to get some evidence for the conjecture that $D(r)=0\left(r^{1 / 2+\epsilon}\right)$ for every $\epsilon>0$ mentioned above.

\section{A Conjecture Refuted by the Study}

During the research on the covering problem the following conjecture, essentially a "law of diminishing" returns," was formulated:

If $\mathrm{C}(\mathrm{n}, \mathrm{r})$ denotes the maximum ratio of coverage attainable with $\mathrm{n}$ disks of radius $\mathrm{r}$, then

$$
C(n+1, r)-C(n, r) \leq C(n, r)-C(n-1, r) .
$$

This means that the successive gains in coverage by the addition of disks one at a time are monotonically nonincreasing. Unfortunately two of the cases that were studied produced results that contradict the conjecture. Table 10 gives the values of the ratio of coverage for the cases involved and the differences. According to the conjecture the second difference should be a negative number, but table 10 shows the two cases found in which it is positive. The amount by which it is positive is large enough so that the error of the approximation could not be responsible for the sign of the second difference.

TABLE 10. Results of several cases showing positive second difference in $\mathrm{C}(\mathrm{n}, \mathrm{r})$

\begin{tabular}{l|r|r|r}
\hline \hline \multicolumn{1}{c|}{ Case } & $\begin{array}{r}\text { Ratio (mesh } \\
\text { of 256) }\end{array}$ & $\begin{array}{c}\text { First differ- } \\
\text { rence }\end{array}$ & $\begin{array}{c}\text { Second diffe- } \\
\text { rence }\end{array}$ \\
\cline { 1 - 2 }$(5,3 / 8)$ & $\begin{array}{r}0.700365 \\
(794168\end{array}$ & $\begin{array}{r}+0.093803 \\
+.101745\end{array}$ & +0.007942 \\
\hline$(7,3 / 8)$ & 895913 & & \\
$(8,5 / 16)$ & .773017 & +.051449 & +.004903 \\
$(9,5 / 16)$ & 824466 & +.056352 & \\
\hline$(10,5 / 16)$ & .880818 & & \\
\hline
\end{tabular}

\section{The Second Covering Problem}

\subsection{Description of Problem}

The second covering problem that was studied is somewhat more complex. We are given a circular area $A$ of radius $R$ and a certain number of straight lines which intersect the area $A$ and divide it into $m$ regions $R_{i}(1 \leq i \leq m)$. In addition, we are given a certain number $n$ of circular disks of radius $r<R$. The problem is to find that placement of the $n$ disks which "covers" the largest amount of the area of $A$ subject to the following restriction:

A point is considered to be covered if and only if it lies inside some disk whose center lies within the same region as the point in question.

For example, the area of $A$ that is covered by the three disks of figure 71 is shaded. Note that the part of disk $C_{1}$ that is in $R_{1}$ is not covered because $C_{1}$ is not centered in $R_{1}$, but rather in $R_{2}$.

\subsection{Analysis of Problem}

A configuration of $n$ disks that maximizes the coverage will necessarily have a given number $n_{i}$ of disks centered in each region $R_{i}$. Furthermore, the placement of the $n_{i}$ disks in $R_{i}$ constitutes the best coverage of $R_{i}$ by $n_{i}$ disks independently of what occurs in other regions.

This observation enables us to separate the problem into two parts and solve it as follows:

(1) For each pair $(i, j)$ subject to $1 \leq i \leq m$ and $1 \leq j \leq n$, calculate the maximum area of region $R_{i}$ that can be covered by $j$ disks. Denote this area by $A(i, j)$.

(2) Let $\Phi$ be the family of $m$-dimensional vectors, $V=\left(n_{1}, n_{2}, \ldots, n_{m}\right)$ such that the $n_{i}$ are all nonnegative integers and $\sum_{i=1}^{m} n_{i}=n$. Now for each $V_{\epsilon} \Phi$ define the sum,

$$
S(V)=\sum_{i=1}^{m} A\left(i, n_{i}\right)
$$

the maximum coverage we seek is then given by

$$
S_{\max }=\operatorname{Max}\{S(V): V \epsilon \Phi\} .
$$

Portion (2) of the solution is a purely combinatorial problem whose solution depends only on the values of the entries in the matrix $A(i, j)$. We shall return to this problem later.

\subsection{Computing $A(i, j)$}

According to the above formulation, the first step in a solution to the problem is the calculation of the matrix $A(i, j)$ for $1 \leq i \leq m, 1 \leq j \leq n$. For a particular $(i, j)$ this means finding the maximum area of a specific region $R_{i}$ that can be covered with $j$ disks. If the region $R_{i}$ were circular, then the calculation would be that of the problem described earlier (sec. 
1). This strong similarity suggested the possibility of using a similar method to calculate $A(i, j)$. As it turned out, only very minor changes were necessary to transform the methods of solving the earlier problem into methods which will compute $A(i, j)$. The changes essentially amount to restricting the centers of disks to grid-points inside $R_{i}$, and not counting points outside $R_{i}$ when computing the coverage of a particular configuration. So far as the computer programs were concerned, these changes were effected with a minimum of difficulty, considering the usual complications which arise in modifying computer codes. The basic reason for this was that the new problem differed from the former only in the region to be covered, and therefore many of the complexities of the program were unchanged.

The program accepts as inputs certain parameters specifying the lines that, along with the boundary of the large circle, form the boundaries of each region $R_{i}$ in question. A line is specified as a "lower slope" or an "upper slope" at input time, according as the region $R_{i}$ lies above or below the line. As is well known, any line in the plane (excepting vertical lines) can be written in the form

$$
y=p x+q .
$$

We assumed further that our lines have rational slopes and $y$-intercepts. That is, $p=p_{1} / p_{2}$ and $q=q_{1} / q_{2}$ where $p_{1}, p_{2}, q_{1}, q_{2}$ are integers with $p_{2}>0, q_{2}>0$. This will be true whenever the line passes through at least two grid-points. ${ }^{27}$ Our equation then can be written as,

or

$$
y=p_{1} x / p_{2}+q_{1} / q_{2}
$$

$$
\left(p_{2} q_{2}\right) y=\left(p_{1} q_{2}\right) x+\left(q_{1} p_{2}\right),
$$

which is of the form

$$
a y=b x+c,
$$

where $a, b, c$ are integers and $a=p_{2} q_{2}>0$. These three integers $a, b, c$ are the input parameters that specify a line $L$. If $L$ were further specified as a lower slope for the region $R_{i}$, then any point $(x, y)$ which lies in $R_{i}$ must satisfy

$$
y \geq p x+q=(b / a) x+(c / a),
$$

that is, $a y \geq b x+c$. It should be noted that this inequality can be tested by the computer using integer arithmetic. This is precisely why we required that $p$ and $q$ be rational. For any $(x, y)$, only a finite number of tests need be made to decide if $(x, y)$ lies in $R_{i}$.

An example of the results of using this program to compute $A(i, j)$ for a particular region $R_{i}(r=3 / 8)$ is given in figures 72 to 75 . The only interesting cases are $j=1,2,3,4$ since total coverage would certainly be possible with five disks of the same radius. Since the computer program is essentially the same as that used in the solution of the former covering prob-

27 Suppose the line $y=p x+q$ goes through points $P_{1}=(r, s)$ and $P_{2}=(t, u)$; then it can be shown easily that $p=(u-s) /(t-r)$ and $q=s-p r$. Therefore if $r, s, t, u$ are integers, then $p$ and $q$ are rational. lem, there seemed no need for an exhaustive examination of cases. The particular difficulties in the second problem may in fact lie in the combinatorial question described in section 8.2.

\subsection{Combinatorial Aspect}

Returning to the second part of the problem described in section 8.2 we find that the combinatorial problem can be reformulated ${ }^{28}$ as an integer linear programing problem. We shall use $a_{i j}$ instead of $A(i, j)$ below, where " $i$ " indexes subregions including a fictitious zeroth subregion to absorb any unused disks. Let the matrix $\left(x_{i j}\right), 0 \leq i \leq M, 0 \leq j \leq N$ be defined as follows:

$x_{i j}=1$ if exactly $j$ disks are allotted to the $i$ th subregion and $x_{i j}=0$ otherwise. We can then express the problem as follows:

Maximize $\Sigma_{i, j} a_{i j} x_{i j}$ subject to constraints
(a) $x_{i j} \geq 0$
$0 \leq i \leq M$
$0 \leq j \leq N$
(b) $\sum_{j=0}^{N} x_{i j}=1$
$0 \leq i \leq M$
(c) $\sum_{i, j} j x_{i j}=N$
(d) $x_{i j}$ an integer
$0 \leq i \leq M$
$0 \leq j \leq N$.

As such, the problem can be handled at least in principle by the methods developed by Gomory [23]. Perhaps an especially effective algorithm can be constructed for the special problem involved here. This remark is added because the general method of Gomory has been found to converge unacceptably slowly in some cases.

\section{Appendix 1. Algorithm and Analysis for Two-Disk Coverage Formula}

\subsection{Description of Parameters}

Suppose given a circle $A$ of radius $r=1$, and two other circles $B$ and $C$, of radius $r<1$, whose centers are at respective distances $d_{1}$ and $d_{2}$ from the center of circle $A$. Let $\theta$ be the angle between

(1) the segment joining the center of $B$ to that of $A$, and

(2) the segment joining the center of $C$ to that of $A$ (see fig. 4).

We construct an algorithm to compute the area ${ }^{29}$ of the "coverage set" $M$ which is common to circle $A$ and at least one of the two circles $B$ and $C$. $M=A \cap(B \cup C)$.

We may assume that the interiors of $B$ and $C$ both meet the interior of $A$ (i.e., both $A \cap B$ and $A \cap C$ are nonempty). Further restrictions on parameters $\left(r, \theta, d_{1}, d_{2}\right)$ are as follows:

(1) $0<r<1$

(2) $d_{1}-r<1 ; d_{2}-r<1$ since $A \cap B$ and $A \cap C$ are nonempty.

28 This integer programing formulation was suggested by A. J. Goldman (NBS Operations Research Section).

${ }_{29}|X|$ shall mean the area of $X$; thus area of $M=|M|$, etc. 

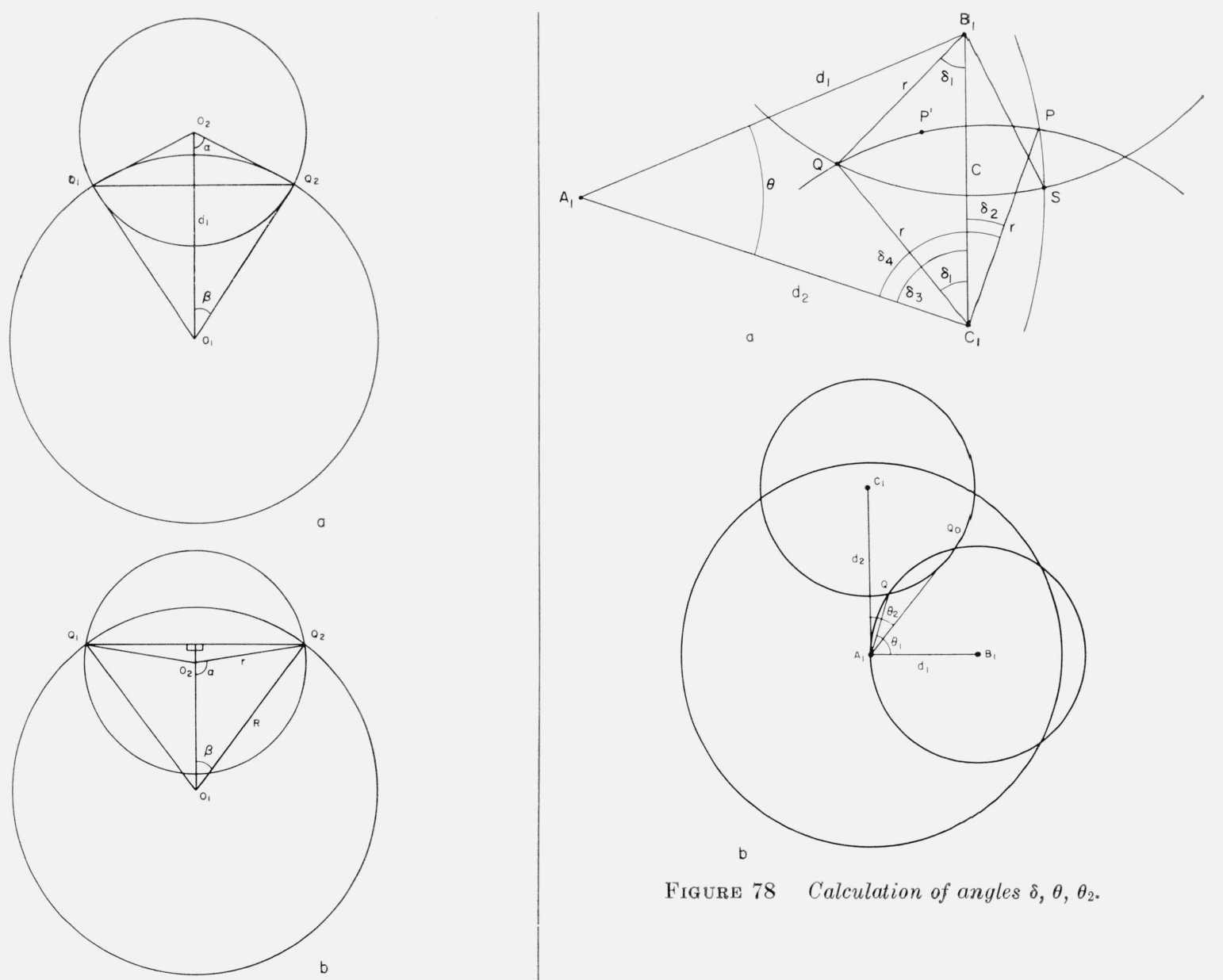

FiguRe 76. Area common to two disks.
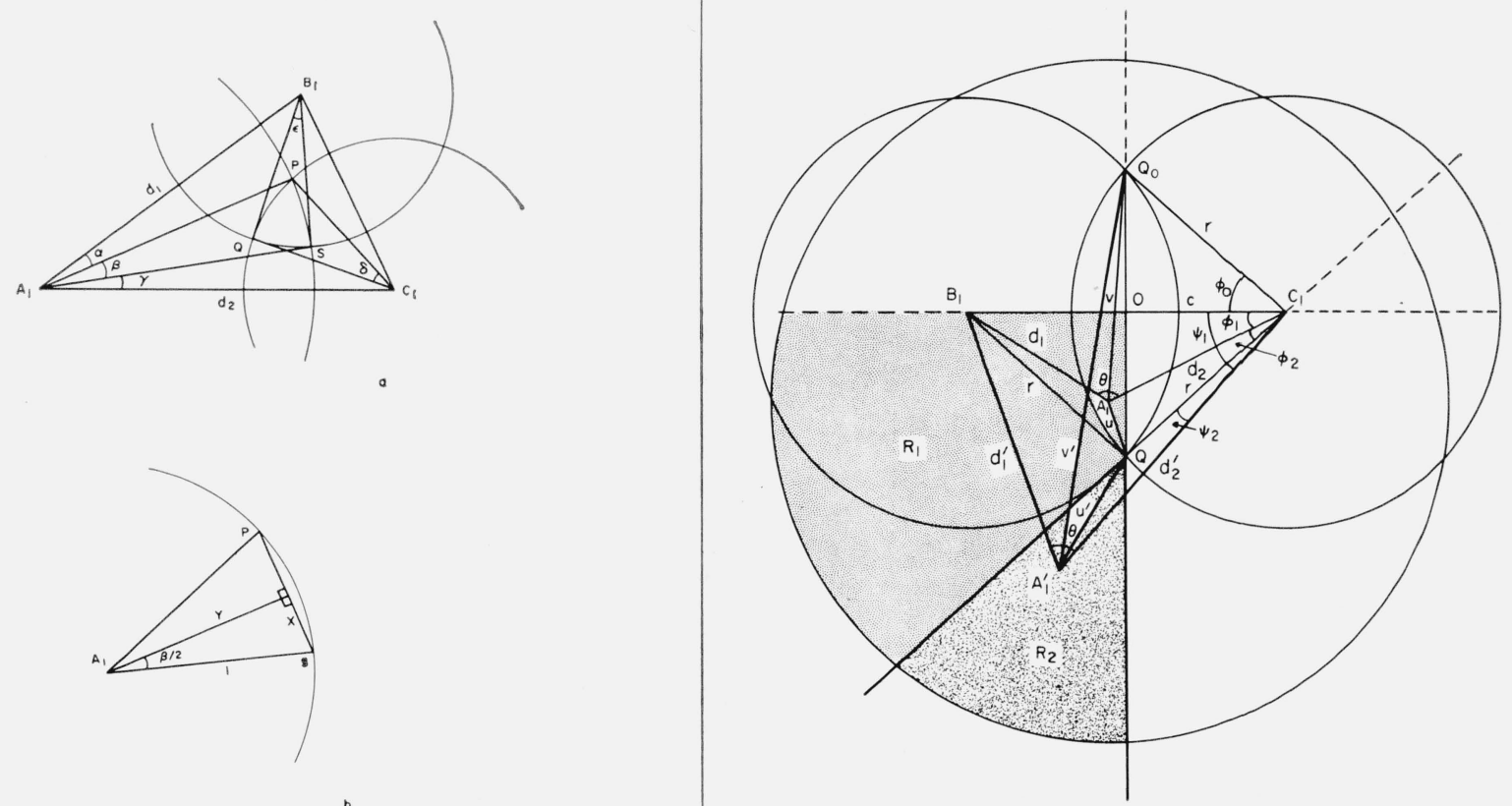

Figure 77. Area common to three disks. 


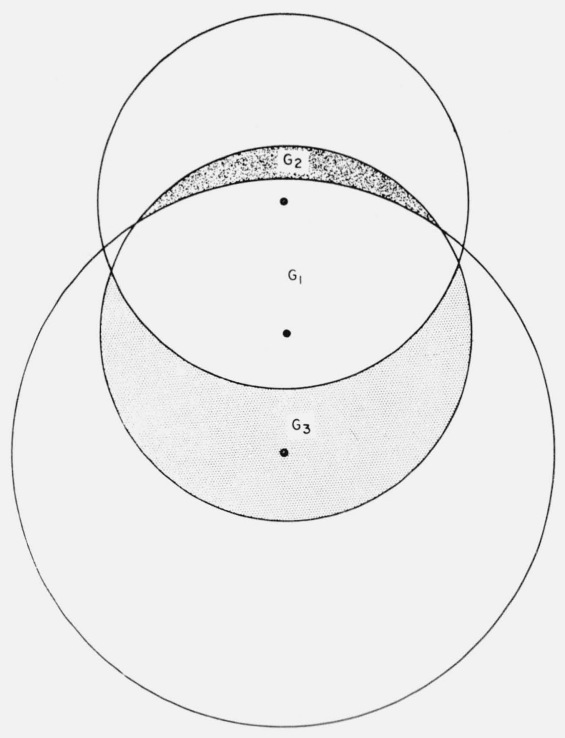

Figure 80. Area bounded by four arcs.

(3) $0 \leq \theta \leq \pi$ since replacing $\theta$ by $(2 \pi-\theta)$ or $(-\theta)$ does not change area.

(4) $d_{1} \leq d_{2}$; we may assume for convenience that $B$ 's center is no further from $A$ 's center than $C$ 's center is.

We shall create an additional parameter $c$ (depending on $\left.\theta, d_{1}, d_{2}\right)$,

$$
c=\left(d_{1}^{2}+d_{2}^{2}-2 d_{1} d_{2} \cos \theta\right)^{1 / 2}
$$

then $c$ is the length of the segment joining the center of $B$ to that of $C$ as can be shown easily from the law of cosines.

There follows an analysis, case by case, of the various configurations that require different treatment when calculating the coverage area in terms of the given parameters $r, \theta, d_{1}, d_{2}$, and the defined parameter $c$.

\subsection{Intersection of Two Disks}

We begin by calculating the area common to two disks of radii $r$ and $R$ respectively with distance of centers $d_{1}<r+R$. In case $\alpha<\pi / 2$ (see fig. 76a) the area may be calculated as follows: ${ }^{30}$

Area $=$ Sector $\left(Q_{1} O_{1} Q_{2}\right)-\Delta Q_{1} O_{1} Q_{2}+$ Sector $\left(Q_{2} O_{2} Q_{1}\right)$ $-\Delta Q_{1} O_{2} Q_{2}$,

Sector $\left(Q_{1} O_{1} Q_{2}\right)=(2 \beta / 2 \pi) \pi R^{2}=R^{2} \beta$,

Sector $\left(Q_{2} O_{2} Q_{1}\right)=(2 \alpha / 2 \pi) \pi r^{2}=r^{2} \alpha$,

$\Delta Q_{1} O_{1} Q_{2}+\Delta Q_{1} O_{2} Q_{2}=2\left(\Delta O_{1} O_{2} Q_{2}\right)=d_{1} R$ sin $\beta$.

We finally get the formula

$$
\text { Area }=R^{2} \beta+r^{2} \alpha-d_{1} R \sin \beta .
$$

30 The symbol $\Delta Q_{1} O_{1} Q_{2}$ means the triangle with these vertices. Sector $\left(Q_{1} O_{1} Q_{2}\right)$ shall denote the area of the sector taken in a clockwise sense from $Q_{1}$ to $Q_{2}$ about
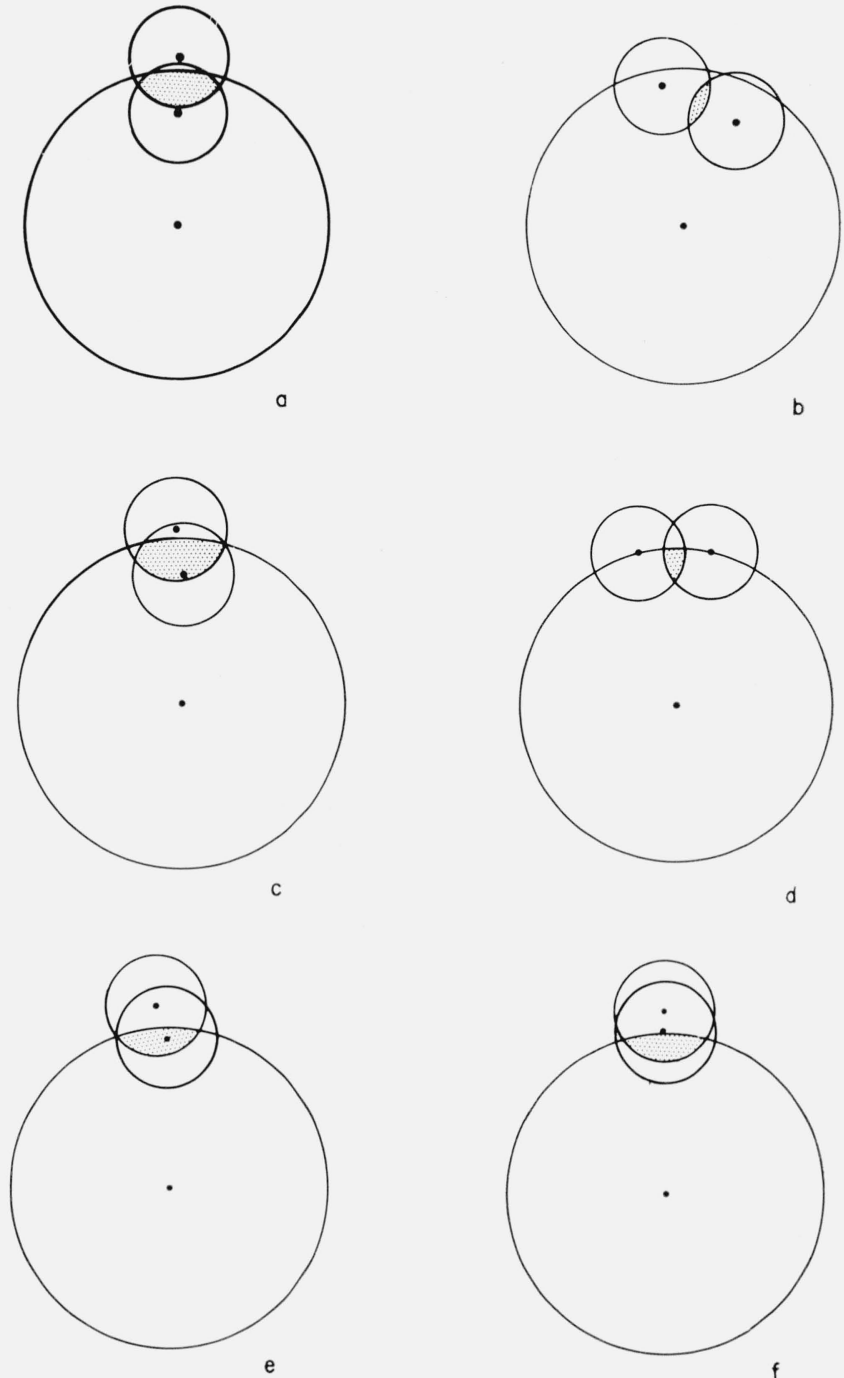

Figure 81. Geometry of two disk cases.

In case $\alpha>\pi / 2$ (see figure $76 \mathrm{~b}$ ) the area is:

Area $=$ Sector $\left(Q_{2} O_{2} Q_{1}\right)+\left(\right.$ Sector $\left(Q_{1} O_{1} Q_{2}\right)$

$\left.-2\left(\Delta O_{1} O_{2} Q_{2}\right)\right)$.

This formula reduces to the same formula as the case $\alpha<\pi / 2$ by a similar argument.

Furthermore in case $\alpha=\pi / 2$ our formula gives Area $=R^{2} \beta+\pi r^{2} / 2-d_{1} R$ sin $\beta$ which is correct also. We have thus shown that for $0<\alpha<\pi$,

$$
\text { Area }=R^{2} \beta+r^{2} \alpha-d_{1} R \sin \beta .
$$

Use of the law of cosines on $\Delta O_{1} O_{2} Q_{2}$ yields

$$
\begin{aligned}
& r^{2}=d_{1}^{2}+R^{2}-2 d_{1} R \cos \beta \\
& R^{2}=d_{1}^{2}+r^{2}-2 r d_{1} \cos \alpha,
\end{aligned}
$$


whence we get $\alpha$ and $\beta$ as

$$
\begin{aligned}
& \alpha=\arccos \left(\left(r^{2}+d_{1}^{2}-R^{2}\right) / 2 r d_{1}\right) \\
& \beta=\arccos \left(\left(R^{2}+d_{1}^{2}-r^{2}\right) / 2 R d_{1}\right) .
\end{aligned}
$$

In particular we notice that if both disks are of equal radius $r$, and $d_{1}=c$, then we get

Area $=2 r^{2}$ arc $\cos (c / 2 r)-c r \sin (\operatorname{arc} \cos (c / 2 r))$.

If $R=1$ and $r<1$, the common area becomes

$$
\text { Area }=\beta+r^{2} \alpha-d_{1} \sin \beta,
$$

where $\alpha$ and $\beta$ are given as

$$
\begin{aligned}
& \alpha=\operatorname{arc} \cos \left(\left(r^{2}+d_{1}^{2}-1\right) / 2 r d_{1}\right), \\
& \beta=\arccos \left(\left(1+d_{1}^{2}-r^{2}\right) / 2 d_{1}\right) .
\end{aligned}
$$

\subsection{Intersection of Three Disks}

We now calculate the area common to three disks where the common area is bounded by three circular arcs (see fig. 77a). In this figure $A_{1}, B_{1}$, and $C_{1}$ represent the respective centers of $A, B$, and $C$ and $P, Q, S$ represent the points of intersection of the three circular arcs. The common area will be calculated by adding together the area of $\triangle P Q S$ and the three areas each bounded by one of the ares and its associated chord. We call these slivers (see fig. 77b).

First we must calculate $\beta$, the angle subtended at $A_{1}$ by the arc PS of disk $A$. Using the law of cosines on $\Delta A_{1} B_{1} S$ and $\Delta A_{1} C_{1} P$, remembering that $\overline{B_{1} S}=r$ $=\overline{C_{1} P}$ and putting ${ }^{31} R=1$, we get

$$
\begin{aligned}
& \alpha+\beta=\arccos \left(\left(d_{1}^{2}-r^{2}+1\right) / 2 d_{1}\right) \\
& \beta+\gamma=\arccos \left(\left(d_{2}^{2}-r^{2}+1\right) / 2 d_{2}\right) .
\end{aligned}
$$

Adding the two equations and subtracting the equation $\alpha+\beta+\gamma=\theta$, we get

$\beta=\arccos \left(\left(d_{1}^{2}-r^{2}+1\right) / 2 d_{1}\right)+\arccos \left(\left(d_{2}^{2}-r^{2}\right.\right.$

$$
\left.+1) / 2 d_{2}\right)-\theta \text {. }
$$

Referring to figure $77 \mathrm{~b}$, we calculate the area of the sliver associated with angle $\beta$ as the difference between the sector $\left(P A_{1} S\right)$ and $\triangle A_{1} P S$. Denoting the area of the sliver as $S_{\beta}$ we have,

$$
\begin{aligned}
S_{\beta} & =\text { Sector }\left(P A_{1} S\right)-\Delta A_{1} P S \\
& =(\beta / 2 \pi) \pi-x y \\
& =\beta / 2-\sin (\beta / 2) \cos (\beta / 2) \\
& =(\beta-\sin \beta) / 2 .
\end{aligned}
$$

We must now calculate the angles $\delta$ and $\epsilon$ of figure 77a. We shall present the argument leading

${ }^{31}$ This assumption involves no loss of generality since the ratio of coverage of $A$ depends only on $(r / R)$. to the calculation of $\delta$; the calculation of $\epsilon$ is similar.

Referring to figure $78 \mathrm{a}$ we wish to calculate the angle $\Varangle Q C_{1} P=\delta_{1}+\delta_{2}=\delta$. Notice that $P$ might lie on the left side of $\overline{B_{1} C_{1}}$, say at $P^{\prime}$, in which case the angle $\delta_{2}$ if measured by $\left(\delta_{4}-\delta_{3}\right)$ would be negative. The formulas we shall derive will not be affected by this difference, as can be readily checked.

We note immediately that

$$
\delta=\delta_{1}+\delta_{4}-\delta_{3}
$$

so that we need only to calculate $\delta_{1}, \delta_{3}, \delta_{4}$.

Using the law of cosines or sines on the proper triangles and noticing that $\triangle Q C_{1} B_{1}$ is isosceles we obtain

$$
\begin{aligned}
\cos \delta_{1} & =(c / 2) / r=c / 2 r, \\
\sin \delta_{3} / d_{1} & =\sin \theta / c, \\
1 & =r^{2}+d_{2}^{2}-2 r d_{2} \cos \delta_{4} .
\end{aligned}
$$

Solving for $\delta_{1}, \delta_{3}, \delta_{4}$ we get

$$
\begin{aligned}
& \delta_{1}=\arccos (c / 2 r) \\
& \delta_{3}=\arcsin \left(d_{1} \sin \theta / c\right) \\
& \delta_{4}=\arccos \left(\left(r^{2}+d_{2}^{2}-1\right) / 2 r d_{2}\right)
\end{aligned}
$$

Substituting into eq (7) we get

$\delta=\operatorname{arc} \cos (c / 2 r)+\arccos \left(\left(r^{2}+d_{2}^{2}-1\right) / 2 r d_{2}\right)$

$$
-\operatorname{arc} \sin \left(d_{1} \sin \theta / c\right) \text {. }
$$

We get a similar expression for $\epsilon$,

$$
\begin{aligned}
\epsilon=\operatorname{arc} \cos \left(\left(r^{2}+d_{1}^{2}-1\right) / 2 r d_{1}\right) & +\operatorname{arc} \cos (c / 2 r) \\
& -\arcsin \left(d_{2} \sin \theta / c\right) .
\end{aligned}
$$

An argument similar to that leading to eq (6) can be used to show that

$$
\begin{aligned}
& \mathrm{S}_{\delta}=r^{2}(\delta-\sin \delta) / 2, \\
& \mathrm{~S}_{\epsilon}=r^{2}(\epsilon-\sin \epsilon) / 2 .
\end{aligned}
$$

Referring to figures $77 \mathrm{a}, \mathrm{b}$ we can readily see that the sides $s_{1}, s_{2}, s_{3}$ of $\triangle P Q S$ are given by

$$
\begin{aligned}
& s_{1}=2 \sin (\beta / 2) \\
& s_{2}=2 r \sin (\delta / 2) \\
& s_{3}=2 r \sin (\epsilon / 2) .
\end{aligned}
$$

The area of $\triangle P Q S$ can then be gotten from the semiperimeter formula

where

$$
\text { Area } \Delta=\sqrt{s\left(s-s_{1}\right)\left(s-s_{2}\right)\left(s-s_{3}\right)}
$$

$$
s=\left(s_{1}+s_{2}+s_{3}\right) / 2 .
$$

In our case $s=\sin (\beta / 2)+r \sin (\delta / 2)+r \sin (\epsilon / 2)$, 
and the common area of the three disks is

Area $=\sqrt{s\left(s-s_{1}\right)\left(s-s_{2}\right)\left(s-s_{3}\right)}$

$$
+(1 / 2)\left(\beta-\sin \beta+r^{2}(\delta-\sin \delta+\epsilon-\sin \epsilon)\right) .
$$

\subsection{Additional Parameters}

In the algorithm which is to follow shortly it is necessary to calculate the distance from $\dot{A}_{1}$, the center of $A$, to the two points, $Q$ and $Q_{0}$, where the disks $B$ and $C$ intersect. We label these points so that $Q$ is the closer to $A_{1}$ and refer to their distances from $A_{1}$ as $u$ and $v$,

$$
u=\overline{A_{1} Q} ; \quad v=\overline{A_{1} Q_{0}} ; \quad u \leq v .
$$

If we consider the disks $B$ and $C$ as already occupying fixed positions in the plane and consider all possible positions of $A_{1}$ we notice almost immediately (referring to fig. 79 ) that $A_{1}$ must lie below $\overline{B_{1} C_{1}}$ and to the left of $\overline{Q Q_{0}}$. This is because $d_{1} \leq d_{2}$ and $u \leq v$. We distinguish two cases.

If $\overline{A_{1}}$ lies in the region $R_{1}$, we have the following equations:

$$
\begin{gathered}
u^{2}=r^{2}+d_{2}^{2}-2 r d_{2} \cos \varphi_{2}, \quad\left(0 \leq \varphi_{2}<\pi / 2\right) \\
\varphi_{1}+\varphi_{2}=\operatorname{arc} \cos (c / 2 r), \quad\left(0 \leq \varphi_{2}<\pi / 2\right) \\
\sin \varphi_{1} / d_{2}=\sin \theta / c
\end{gathered}
$$

and these can be solved for the value of $u^{2}$ as:

$u^{2}=r^{2}+d_{2}^{2}-2 r d_{2} \cos \lceil\arccos (c / 2 r)$

$$
\left.-\arcsin \left(d_{1} \sin \theta / c\right)\right] \text {. }
$$

If $A_{1}$ lies in region $R_{2}$, say at $A_{1}^{\prime}$ in the figure, then we get the equations:

$$
\begin{gathered}
u^{2}=r^{2}+d_{2}^{2}-2 r d_{2} \cos \varphi_{2}, \quad\left(0 \leq \varphi_{2}<\pi / 2\right) \\
\varphi_{1}-\varphi_{2}=\operatorname{arc} \cos (c / 2 r), \quad\left(0 \leq \varphi_{1}<\pi / 2\right) \\
\sin \varphi_{1} / d_{1}=\sin \theta / c
\end{gathered}
$$

and these can be solved for the value of $u^{2}$ as:

$u^{2}=r^{2}+d_{2}^{2}-2 r d_{2} \cos \left[\operatorname{arc} \sin \left(d_{1} \sin \theta / c\right)\right.$

$$
-\operatorname{arc} \cos (c / 2 r)] \text {. }
$$

We notice that the expressions in brackets in eqs (18) and (19) differ only in sign and since $\cos x$ is an even function $(\cos (-x)=\cos x)$ the formulas for $u^{2}$ are identical.

In the first case $\left(A_{1}\right.$ in $\left.R_{1}\right)$ we have the following expressions

$$
v^{2}=r^{2}+d_{2}^{2}-2 r d_{2} \cos \left(\varphi_{0}+\varphi_{1}\right)
$$

where $\varphi_{0}$ is given by

$$
\varphi_{0}=\operatorname{arc} \cos (c / 2 r)
$$

and $\varphi_{1}$ is given by

$$
\varphi_{1}=\arcsin \left(d_{1} \sin \theta / c\right)
$$

In the second case $\left(A_{1}\right.$ in $\left.R_{2}\right)$ we have

$$
v^{2}=r^{2}+d_{2}^{2}-2 r d_{2} \cos \left(\varphi_{0}+\psi_{1}\right)
$$

where $\psi_{1}$ is given by

$$
\psi_{1}=\arcsin \left(d_{1} \sin \theta / c\right)
$$

and $\phi_{0}$ is given by (21).

Once again the formulas for $v^{2}$ are identical so there is no need to make a distinction between the cases.

Recalling that we are assuming $R=1$, the situation that $Q$ lies inside $A$ is expressed by $u \leq 1$ or equivalently $u^{2} \leq 1$. Similarly $Q_{0}$ lies inside $\bar{A}$ if and only if $v^{2} \leq 1$. Thus we have a perfectly effective test for this situation.

\subsection{Analysis of Cases}

We suppose throughout that both disks $B$ and $C$ meet the large circle $A$, and that $B$ is at least as close as $C$ to the center of $A$. We also assume that the radius $r$ of $B$ and $C$ is less than that of $A$ and that $\theta$ measures the smaller angle formed at the center of $A$. These assumptions are equivalent to the algebraic restrictions on parameters contained in (1), (2), (3), and (4) of this appendix 1, section 9.2.

Case $F_{1}$ : Both disks are entirely within $A$. This is the case when

$$
d_{2}+r \leq 1 .
$$

This says that disk $C$ is inside $A$, but we agreed that $B$ was at least as close in, so both must lie inside. We distinguish two subcases.

Subcase $F_{11}$ : $B$ and $C$ overlap. This is true when ${ }^{32}$

$$
c<2 r,
$$

and the formula for the common area covered is

$$
\text { Area }=2 \pi r^{2}-|B \cap C| \text {. }
$$

where $|B \cap C|$ is found according to eq (2).

Subcase $F_{12}$ : $B$ and $C$ do not overlap. This is true when

$$
c \geq 2 r
$$

and the formula for the area covered is

$$
\text { Area }=2 \pi r^{2} .
$$

Case $F_{2}: B$ is entirely inside $A, C$ partially so. This is true when $d_{1}+r \leq 1<d_{2}+r$. Again we distinguish two subcases.

Subcase $F_{21}: B$ and $C$ overlap. The condition is (26) and the area is given by

$$
\text { Area }=\pi r^{2}+|A \cap C|-|B \cap C|,
$$

32 The quantity $c$ denotes the distance of centers of $B$ and $C$ and is calculated by $c=\left(d_{1}^{2}+d_{2}^{2}-2 d_{1} d_{2} \cos \theta\right)^{1 / 2}$. 
where $|A \cap C|$ is given by (3), (4), (5) with $d_{2}$ replacing $d_{1}$ in all three formulas for obvious reasons. $|B \cap C|$ is given by (2).

Subcase $F_{22}: B$ and $C$ do not overlap. The condition is (28) and the formula for the area is

$$
\text { Area }=\pi r^{2}+|A \cap C| \text {. }
$$

Case $F_{3}$ : Both $B$ and $C$ are only partially inside $A$ and they overlap. The condition is (26) and $d_{1}+r>1$.

We now consider in order the subcases of $\mathrm{F}_{3}$ beginning with the case $\theta=\pi$ and the case $c=0$. We eliminate the possibility $c=0$ early in the game since we call for division by $c$ in many cases.

Subcase $F_{30}$ : The centers of $B, A$, and $C$ are collinear in the order indicated so that the condition is $\theta=\pi$; it can be easily established that in this case $u<1$ necessarily and that the common region $B \cap C$ lies entirely within $A$. The area is then given by

$$
\text { Area }=|A \cap B|+|A \cap C|-|B \cap C| .
$$

Subcase $F_{31}: B$ and $C$ are coincident. The conditions are

$$
\theta=0 ; \quad d_{1}=d_{2}
$$

and the area is given by

$$
\text { Area }=|A \cap B|,
$$

which is calculated by eqs (3), (4), (5).

Subcase $F_{32}$ : The centers of $A, B$, and $C$ are collinear, but $B$ and $C$ are not coincident, and the two intersection points of disks $B$ and $C$ lie inside $A$. The conditions are

$$
\theta=0 ; \quad d_{1} \neq d_{2} ; \quad u^{2}<1
$$

where $u^{2}$ is given by

$$
u^{2}=r^{2}+d_{1} d_{2} .
$$

It can be readily verified that eq (18) reduces to (36) under the conditions of (35). The area is given by

$$
\text { Area }=\pi r^{2}+|A \cap C|-|B \cap C| .
$$

Subcase $F_{33}$ : The same conditions hold as for $F_{32}$ except the two intersection points of $B$ and $C$ lie outside $A$. The condition is

$$
u^{2}=r^{2}+d_{1} d_{2} \geq 1
$$

and the area is given by

$$
\text { Area }=|A \cap B| \text {. }
$$

In the following situations we shall describe the cases according to the conditions on the parameters and let the reader figure out the geometry for himself. First we define two new parameters $\theta_{1}$ and $\theta_{2}$.

$$
\theta_{i}=\operatorname{arc} \cos \left(\left(1+d_{i}^{2}-r^{2}\right) / 2 d_{i}\right) \quad i=1,2 .
$$

Referring to figure $78 b, \theta_{1}$ is the angle $\Varangle Q A_{1} B_{1}$ and $\theta_{2}$ the angle $\Varangle C_{1} A_{1} Q_{0}$.

Subcase $F_{34}$ : The conditions are ${ }^{33}$

$$
\theta \neq 0 ; \quad u^{2}<1 ; \quad v^{2}<1 ; \quad \theta<\theta_{2}
$$

and the area is given by

$$
\text { Area }=\pi r^{2}+|A \cap C|-|B \cap C| .
$$

Subcase $F_{35}$ : The conditions are ${ }^{34}$

$$
\theta \neq 0 ; \quad u^{2}<1 ; \quad v^{2}<1 ; \quad \theta>\theta_{2}
$$

and the area is given by

$$
\text { Area }=|A \cap B|+|A \cap C|-|B \cap C| .
$$

Subcase $F_{36}$ : The conditions are

$$
u^{2}<1 ; \quad v^{2}>1
$$

and the area is given by

$$
\text { Area }=|A \cap B|+|A \cap C|-|B \cap C \cap A|
$$

where the last term is the common area of the triangular region whose area is calculated in section 9.3 of appendix 1 , eq (14).

Subcase $F_{37}$ : The conditions are

$$
u^{2}<1 ; \quad v^{2}=1 ; \quad \theta<\theta_{2}
$$

and the area is given by

$$
\text { Area }=|A \cap B|+|A \cap C|-|B \cap C \cap A| .
$$

Subcase $F_{38}$ : The conditions are

$$
u^{2}<1 ; \quad v^{2}=1 ; \quad \theta>\theta_{2}
$$

and the area is given by

$$
\text { Area }=|A \cap B|+|A \cap C|-|B \cap C| .
$$

Subcase $F_{39}$ : The conditions are

$$
u^{2}=1 ; \quad v^{2}=1
$$

and the area is given by

$$
\text { Area }=|A \cap B| \text {. }
$$

Subcase $F_{40}$ : The conditions are

$$
u^{2}=1 ; \quad v^{2}>1 ; \quad \theta<\theta_{1}
$$

and the area is given by

$$
\text { Area }=|A \cap B| \text {. }
$$

${ }^{33}$ See section 9.6 of appendix 1 for an explanation of the inequalities on the $\theta_{i}$. See section 9.4 of appendix 1 for definitions of $u$ and $v$.

${ }_{34} \theta \neq 0$ will be true for all cases $F_{34}$ through $F_{43}$. 
Subcase $F_{41}$ : The conditions are

$$
u^{2}=1 ; \quad v^{2}>1 ; \quad \theta>\theta_{1}
$$

and the area is given by

$$
\text { Area }=|A \cap B|+|A \cap C| .
$$

Subcase $F_{42}$ : The conditions are

$$
u^{2}>1 ; \quad v^{2}>1 ; \quad \theta<\theta_{1}
$$

and the area is given by

$$
\text { Area }=|A \cap B| .
$$

Subcase $F_{43}$ : The conditions are

$$
u^{2}>1 ; \quad v^{2}>1 ; \quad \theta>\theta_{1}
$$

and the area is given by

$$
\text { Area }=|A \cap B|+|A \cap C| \text {. }
$$

The final case occurs when both disks $B$ and $C$ meet $A$ but not each other.

Case $F_{44}$ : The conditions are

$$
d_{2}+r>1 ; \quad d_{1}+r>1 ; \quad c \geq 2 r
$$

and the area is given by

$$
\text { Area }=|A \cap B|+|A \cap C| \text {. }
$$

\subsection{Special Cases}

Most of the formulas for the area covered that are presented in section 9.5 of appendix 1 can be verified by a consideration of the geometry of the configurations. An exception to this is the formula $\pi r^{2}+|A \cap C|-|B \cap C|$ which appears in cases $F_{32}$ and $F_{34}$.

Referring to figure 80 and denoting the areas indicated as $G_{1}, G_{2}$, and $G_{3}$ we get the following expression for the area covered:

$$
\text { Area }=|A \cap C|+|A \cap B|-G_{1}
$$

but we also have the equations

$$
\begin{aligned}
G_{1}+G_{2}+G_{3} & =\pi r^{2} \\
G_{1}+G_{2} & =|B \cap C| \\
G_{1}+G_{3} & =|A \cap C| .
\end{aligned}
$$

Solving for $G_{1}$ and substituting in (63) we get

$$
\text { Area }=|A \cap C|-|B \cap C|+\pi r^{2} .
$$

As regards the inequalities on the $\theta_{i}$, if $\theta<\theta_{2}$ then the portion of disk $B$ that lies outside $A$ is inside $C$. If $\theta>\theta_{2}$ then this portion does not meet $C$ and the area is calculated accordingly. Figures 81a and $81 \mathrm{~b}$ refer respectively to cases $\mathrm{F}_{34}$ and $\mathrm{F}_{35}$ and the inequalities can be seen geometrically. The inequalities on $\theta$ and $\theta_{1}$ are similarly motivated.

\section{Appendix 2. Analytical Solution ${ }^{35}$ for $N=2$}

This section deals with the maximization of $F(X)$ in the very simple case $n=2$. As noted in the main text, and explained in detail in appendix $1, F(X)$ is given by one of eight different formulas, depending on the nature of the configuration formed by the fixed circle $A$ of radius $R=1$ (this is the circle "to be covered") and the two "covering circles" $C_{1}$ and $C_{2}$ of radius $r<1$. Despite this complication, we shall show that the problem can be solved analytically.

To a void trivial cases, the assumption $1 / 2<r<1$ will be made throughout. As in appendix 1, the following notation will be used:

$d_{1}=$ distance from $C_{1}$ 's center to $A$ 's center,

$d_{2}=$ distance from $C_{2}^{\prime}$ 's center to $A$ 's center,

$c=$ distance between $C_{1}$ 's center and $C_{2}$ 's center, $\theta=$ angle between radius of $A$ through $C_{1}$ 's center and that through $C_{2}$ 's center.

Thus we have, by the Law of Cosines,

$$
c^{2}=d_{1}^{2}+d_{2}^{2}-2 d_{1} d_{2} \cos \theta .
$$

The function to be maximized is given, in set theoretic notation, by

$$
\begin{aligned}
& \pi F(X)=G\left(d_{1}, d_{2}, \theta\right)=\text { Area }\left(A \cap C_{1}\right) \\
& + \text { Area }\left(A \cap C_{2}\right)-\text { Area }\left(A \cap C_{1} \cap C_{2}\right) .
\end{aligned}
$$

A preliminary remark which greatly simplifies the situation is that $\theta=\pi$ for any configuration which maximizes $F(X)$. To prove this, temporarily regard $d_{1}$ and $d_{2}$ as fixed, but $\theta$ as variable. That is, regard $C_{1}$ as fixed but $C_{2}$ as rotatable around the center of $A$. Then the first two areas in the right-hand side of eq (2) are constant, but the third area is a decreasing function of $C$ and therefore (cf.eq (1)) is a decreasing: function of $\theta$ for $0 \leq \theta \leq \pi$ and an increasing function of $\theta$ for $\pi \leq \theta \leq 2 \pi$.

In what follows, therefore, $\theta=\pi$ will be assumed, so that eqs (1) and (2) become, respectively,

$$
\begin{gathered}
c=d_{1}+d_{2}, \\
G\left(d_{1}, d_{2}, \pi\right)=g\left(d_{1}, d_{2}\right)=\text { Area }\left(A \cap C_{1}\right) \\
+ \text { Area }\left(A \cap C_{2}\right)-\text { Area }\left(C_{1} \cap C_{2}\right),
\end{gathered}
$$

where eq (4) follows from the observation that $A \cap C_{1} \cap C_{2}=C_{1} \cap C_{2}$ when $\theta=\pi$.

Next it will be shown that, for every configuration maximizing $F(X)$,

$$
\begin{gathered}
d_{1}+d_{2}=c \leq 2 r, \\
d_{i}+r \geq 1 \quad i=1,2 .
\end{gathered}
$$

35 This solution is due to B. K. Bender and A. J. Goldman (NBS Operations Research Section). C.T. Zahn, Jr., suggested several expository improvements. 
Condition (5) asserts that $C_{1}$ and $C_{2}$ meet, while condition (6) asserts that $C_{1}$ and $C_{2}$ "stick out" past $A$, or at least are not entirely interior to $A$.

For the proof, first assume tentatively that $c>2 r$ for some configuration which maximizes $F(X)$. Then $d_{i}+r \leq 1$ cannot hold for both $i=1$ and $i=2$, since this would imply

$$
4 r<c+2 r=\left(d_{1}+r\right)+\left(d_{2}+r\right) \leq 2,
$$

contradicting the hypothesis $r>1 / 2$. Thus at least one of the sunctions Area $\left(A \cap C_{i}\right)(i=1,2)$ is a strictly decreasing function of $d_{i}$ near the configuration in question. For such an $i$, we can slightly decrease $d_{i}$ and thus increase Area $\left(A \cap C_{i}\right)$ without violating the condition $c>2 r$. Thus one of the first two areas in eq (4) is increased, the other is unchanged, and the third remains zero since $C_{1}$ and $C_{2}$ are disjoint when $c>2 r$. Therefore $g\left(d_{1}, d_{2}\right)$ has been increased, violating the assumption that the original configuration was maximizing. So the tentative assumption that $c>2 r$ is untenable, i.e., condition (5) holds for every maximizing configuration.

Now temporarily regard $c$, and thus the third area in eq (4), as fixed, so that eq (3) is a constraint on $d_{1}$ and $d_{2}$. For any configuration in which $d_{1}+r>1$ but $d_{2}+r<1$, it would be possible to decrease $d_{1}$ slightly (thus increasing Area $\left(A \cap C_{1}\right)$ ) and to increase $d_{2}$ by the same amount so that $d_{2}+r<1$ is not violated and Area $\left(A \cap C_{2}\right)$ retains the value $\pi r^{2}$. Thus $g\left(d_{1}, d_{2}\right)$ would be increased, and so the original configuration could not have been maximizing. A similar argument applies with $i=1$ and $i=2$ interchanged. Therefore a maximizing configuration either obeys (6) for $i=1,2$, or obeys

$$
d_{i}+r \leq 1 \quad \text { for } i=1,2 .
$$

(6a)

Under the condition (6a), however, the first two areas in eq (4) have the value $\pi r^{2}$ and only the third one is variable. This area is minimized (i.e., $g\left(d_{1}, d_{2}\right)$ is maximized) by choosing $d_{1}$ and $d_{2}$ (and thus $c=d_{1}+d_{2}$ ) as large as possible. Subject to (6a) these choices are $d_{i}-1-r(i=1,2)$, which still satisty (5) since

$$
c=2-2 r \leq 2 r \text { because } r>1 / 2 .
$$

But these choices also obey (6). This completes the proof that (5) and (6) hold for all configurations maximizing $F(X)$.

In what follows, therefore, conditions (5) and (6) will be assumed. It is convenient to introduce the following quantities:

$2 \theta_{i}=$ angle intercepted at $A$ 's center by subtended arc of $C_{i}$,

$2 \varphi_{i}=$ angle intercepted at $C_{i}$ 's center by subtended arc of $A$,

$z_{i}=$ length of common chord of $A$ and $C_{i}$,

$2 \psi=$ angle intercepted at center of either $C_{1}$ or $C_{2}$ by subtended arc of the other of $C_{1}$ or $C_{2}$,

$z=$ length of common chord of $C_{1}$ and $C_{2}$.
It is readily found that

$$
\begin{aligned}
& \cos \theta_{i}=\left(1-r^{2}+d_{i}{ }^{2}\right) / 2 d_{i}, \\
& \cos \theta_{i}=\left(1-r^{2}-d_{i}{ }^{2}\right) / 2 r d_{i}, \\
& \cos \psi=c / 2 r \\
& \sin \theta_{i}=r \sin \phi_{i}=\frac{1}{2} z_{i}, r \sin \psi=\frac{1}{2} z,
\end{aligned}
$$

Area $\left(A \cap C_{i}\right)=\pi r^{2}+\theta_{i}-\frac{1}{2} \sin 2 \theta_{i}-r^{2} \varphi_{i}+\frac{1}{2} r^{2} \sin 2 \varphi_{i}$,

Area $\left(C_{1} \cap C_{2}\right)=2 r^{2} \psi-r^{2} \sin 2 \psi$.

From the geometry of the situation, it follows that

$$
\text { о }\left(\text { Area }\left(A \cap C_{i}\right)\right) / \partial\left(d_{i}\right)=-z_{i} ;
$$

an analytical derivation of this will be given later. Exactly the same argument shows that

$$
\partial\left(\text { Area }\left(C_{1} \cap C_{2}\right)\right) / \partial d_{i}=-z,
$$

so that (see eq (4)) we have

$$
\partial g / \partial d_{i}=z-z_{i} \quad(i=1,2) .
$$

Now it will be shown that there is precisely one maximizing configuration, the one characterized rather elegantly by

$$
z_{1}=z_{2}=z
$$

or equivalently, via eq (10), by

$$
\varphi_{1}=\varphi_{2}=\psi
$$

Note that, as might be expected, the maximizing configuration is symmetric in the sense that $d_{1}=d_{2}$.

To prove eq (15), tentatively suppose it false. Without loss of generality suppose $z_{1} \neq z$. Then the function $g\left(d_{1}, d_{2}\right)$ assumes its maximum on the triangle:

$$
T: d_{1} \geq 1-r, \quad d_{2} \geq 1-r, \quad d_{1}+d_{2} \leq 2 r,
$$

defined in the $\left(d_{1}, d_{2}\right)$ - plane by conditions (5) and (6), at a point at which $\partial g / \partial d_{1}$ does not vanish. Such a point must lie on the boundary of $T$, and in fact not on its horizontal leg (endpoints excluded); this follows from standard calculus arguments. If the point is on the vertical leg of $T$ (upper end-point excluded), then on the one hand $\partial g / \partial d_{1}$ must be nonpositive and thus negative, so that $z<z_{1}$ by eq (14), and on the other hand $d_{1}+r=1$ so that $C_{1}$ is internally tangent to $A$, implying $z_{1}=0$. Since $z<z_{1}$ and $z_{1}=0$ are incompatible, this case is ruled out. If the point is on the hypotenuse of $T$ (upper endpoint excluded), then on the one hand $\partial g / \partial d_{1}$ must be nonnegative and thus positive, so that $z>z_{1}$, and on the other hand $c=2 r$ so that $C_{1}$ and $C_{2}$ are internally tangent, implying $z=0$. Since $z>z_{1}$ and $z=0$ are incompatible, this case is also ruled out. Finally, if the point is the upper vertex of $T$, then on the one hand consideration of the directional derivative 
along the hypotenuse of $T$ yields

$$
\partial g / \partial d_{1}-\partial g / \partial d_{2}=\left(z-z_{1}\right)-\left(z-z_{2}\right)=z_{2}-z_{1} \leq 0,
$$

while on the other hand $z_{1}=0$ and $z=0$ as above. This implies $z_{2}=0$, so that $C_{1}$ and $C_{2}$ are externally tangent to each other and internally tangent to $\dot{A}$. Such a configuration can only occur if $r=1 / 2$, contradicting our assumption that $r>1 / 2$. So every alternative to eq (15) has been ruled out, and the equation must hold. To describe the maximizing configuration more explicitly, let $x$ denote the common value of $d_{1}$ and $d_{2}$. Equations (8), (9), and (16) then yield

$$
\left(1-r^{2}-x^{2}\right) / 2 r x=x / r,
$$

which implies that

$$
x=\left(\left(1-r^{2}\right) / 3\right)^{1 / 2}
$$

As a check, note that $x \rightarrow 1 / 2$ as $r \rightarrow 1 / 2$ and $x \rightarrow 0$ as $r \rightarrow 1$, as would be anticipated.

The maximum coverage ratio $F_{\max }$ can now be found in terms of $r$. First we have, from eqs (4), (11), (12), and (16)

$$
\pi F_{\max }=2 \pi r^{2}+\left(2 \theta_{i}-4 r^{2} \psi\right)-\sin 2 \theta_{i}+2 r^{2} \sin 2 \psi .
$$

From eqs (7) and (18), however,

$$
\cos \theta_{i}=2\left(\left(1-r^{2}\right) / 3\right)^{1 / 2}=2 x,
$$

which with the aid of eqs (9), (10), and (16) yields $2 r^{2} \sin 2 \psi=4 r^{2} \sin \varphi_{i} \cos \psi=4 r\left(\sin \theta_{i}\right)(x / r)=$

$$
2 \sin \theta_{i} \cos \theta_{i}=\sin 2 \theta_{i} .
$$

Thus the last two terms in the above expression for $\pi F_{\max }$ cancel each other, leading to

\begin{tabular}{|c|c|c|c|c|}
\hline$r$ & $x$ & $x_{c}$ & $F_{\max } / \pi$ & $\mathrm{RATIO}_{\max }$ \\
\hline $\begin{array}{l}9 / 16 \\
5 / 8 \\
11 / 16 \\
344 \\
1316 \\
7 / 8 \\
15 / 16\end{array}$ & $\begin{array}{r}0.47735 \\
.45069 \\
.41926 \\
.38188 \\
.33657 \\
.27951 \\
.20091\end{array}$ & $\begin{array}{r}\text { b } 0.47754 \\
.45069 \\
.41753 \\
.38181 \\
.33802 \\
.28128\end{array}$ & $\begin{array}{r}0.601 \\
.686 \\
.762 \\
.829 \\
.889 \\
.939 \\
.979\end{array}$ & $\begin{array}{r}0.600 \\
.686 \\
.732 \\
.829 \\
.889 \\
.939 \\
.979\end{array}$ \\
\hline
\end{tabular}

$$
\pi F_{\max }=2 \pi r^{2}+2 \arccos (2 x)-4 r^{2} \operatorname{arc} \cos (x / r),
$$

where $x$ is given by (18).
TABLE 11. Comparison of analytic and computer solutions ${ }^{a}$ for the case $\mathrm{n}=2$

a $x$ is the distance of the centers of each of $C_{1}$ and $C_{2}$ from that of $A$ in the optimal configuration, and $F_{\max } / \pi$ is the value of coverage obtained from the configuration, i.e., the maximum coverage. The corresponding values $x_{c}$ and RATIO Rax $_{a x}$ are those obtained from the computer simulation at a mesh of 256 .

$b$ At the coarser mesh of 64 , the value is $x_{c}=0.476$

The value of $x$ and maximum coverage $\left(F_{\max } / \pi\right)$ are compared in table 11 with the corresponding values $x_{c}$ and RATIO max obtained by the computer. ${ }^{36}$ As can be seen, the agreement is excellent. 13).

We conclude with an analytical derivation of eq

First use eq (11) to write

ว $\left(\right.$ Area $\left.\left(A \cap C_{i}\right)\right) / \partial\left(d_{i}\right)=2 \sin ^{2} \theta_{i}\left(\partial \theta_{i} / \partial\left(d_{i}\right)\right)$

$$
-2 r^{2} \sin ^{2} \varphi_{i}\left(\partial \varphi_{i} / \partial\left(d_{i}\right)\right)
$$

By eq (10), this can be written

ว $\left(\right.$ Area $\left.\left(A \cap C_{i}\right)\right) / \partial\left(d_{i}\right)=z_{i} \sin \theta_{i}\left(\partial \theta_{i} / \partial\left(d_{i}\right)\right)$

$$
-r \sin \varphi_{i}\left(\partial \varphi_{i} / \partial\left(d_{i}\right)\right)
$$

From eqs (7) and (8), however,

$$
\begin{gathered}
-\sin \theta_{i}\left(\partial \theta_{i} / \partial\left(d_{i}\right)\right)=-\left(1-r^{2}-d_{i}{ }^{2}\right) / 2 d_{i}{ }^{2}, \\
-r \sin \varphi_{i}\left(\partial \varphi_{i} / \partial\left(d_{i}\right)\right)=-\left(1-r^{2}+d_{i}{ }^{2}\right) / 2 d_{i}{ }^{2} .
\end{gathered}
$$

Substitution of these results into eq (20) yields eq (13).

${ }^{30}$ This table was prepared by C. T. Zahn, Jr. 


\section{Appendix 3. The FORTRAN Program}

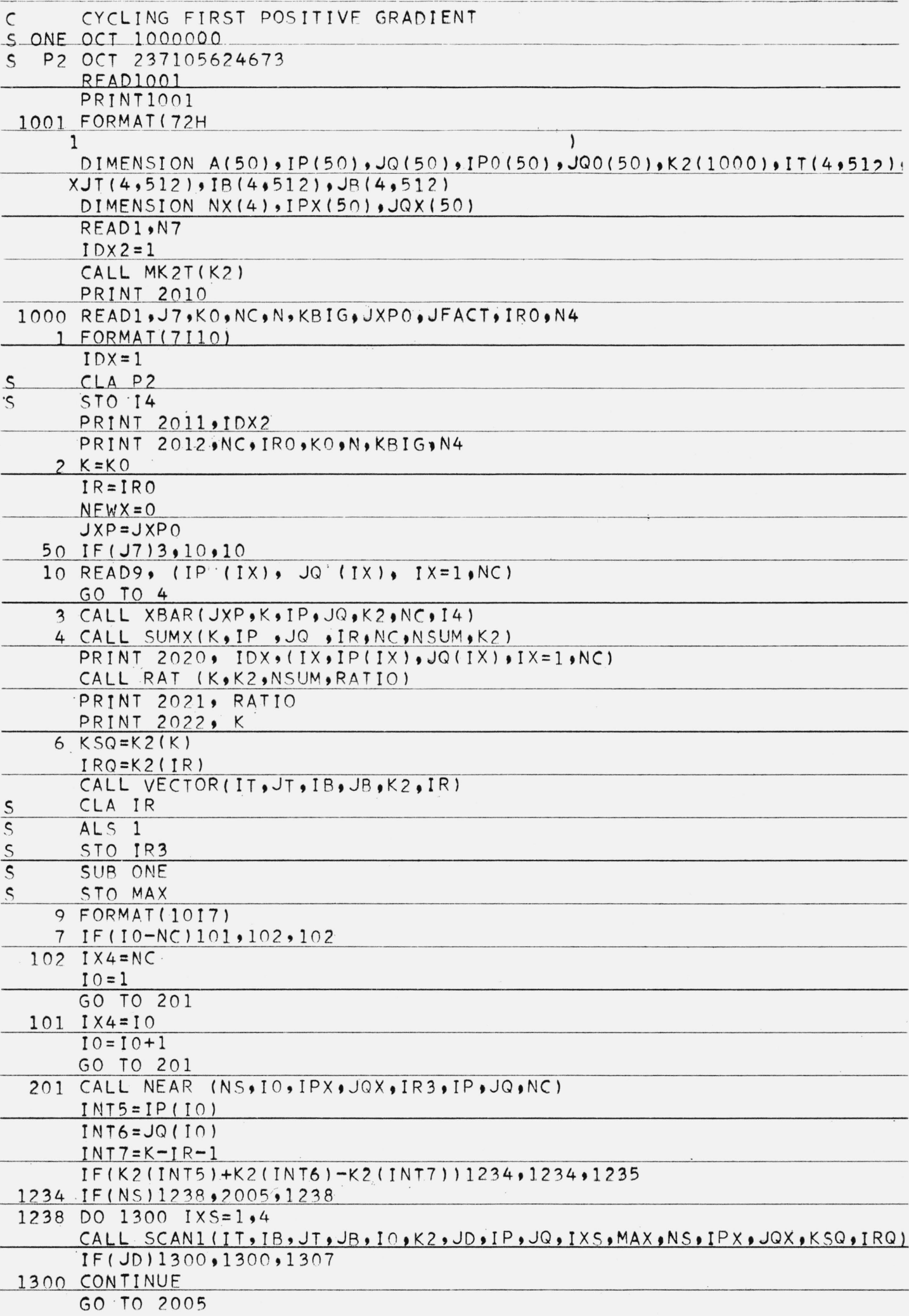


1235 DO $1250 \quad$ IXS $=1,4$

CALL SCAN2 (IT, IB, JT, JB, I , K2, JD, IP, JQ, IXS, MAX,NS, IPX, JQX,KSQ, I RQ)

IF ( JD) $1250,1250,1307$

1250 CONTINUF

2005 IF $(I 0-I \times 4) 304,303,302$

302 IF (IO-NC) $304,305,305$

$305 \quad 10=1$

GO TO 201

$30410=10+1$

GO TO 201

303 IF $(K-K B I G) 18,10,19$

1307 I $X 0=10$

$M \times 2=I \times S$

CALL XNEW $(M \times 2, I P, J Q, I X 0)$

NEWX $=N E W X+1$

GO TO 7

18 PRINT 2027, NEWX

PRINT $2030, K$, (IX, IP(IX),JQ(IX),IX $=1, N C$ )

CALL SUMX(K,IP,JQ,IR,NC,NSUM,K2)

CALL RAT (K,K2,NSUM,RATIO)

PRINT 2021, RATIO

CALL REFINE (IT,JT,IB,JB,NC,K,IR,IP,JQ,JXP,JFACT)

PRINT 2032, K

GO TO 6

19 CALL SUMX (K,IP,JQ,IR,NC,NSUM,K2)

CALL RAT (K,K2,NSUM,RATIO)

PRINT $2040, K,($ IX, IP (IX), JQ(IX), IX $=1, N C)$

PRINT 2041, RATIO

PRINT 2042, NEWX

53 IF(IDX-N4) 41,42,42

4. IDX $=$ IDX+1

GO TO 2

42 IF (IDX2-N7) 43,44, 44

44 CALL RETURN

43 ID $2=10 \times 2+1$

GO TO 1000

2010 FORMAT ( $1 \mathrm{HI}, 19 \mathrm{X}, 38 \mathrm{HCYCLING}$ FIRST POSITIVE GRADIENT SEARCH)

2011 FORMAT $(1 \mathrm{HI}, 19 \times, 5 \mathrm{HCASE}, \mathrm{I} 2 / 1)$

2012 FORMAT $120 \mathrm{X}, 1$ OHTHERE ARE, I $2,26 \mathrm{H}$ COVERING DISCS OF RADIUS, I3,1H/, I $\times 3 / 20 \times, 25 H M O N T E$ CARLO IS USED WITH, I $3,8 \mathrm{H}$ TRIALS $\bullet / 20 X, 19 \mathrm{HFINAL} \mathrm{MESH}$ $X$ SIZF IS, I4, I3H. KICK OFF, I3,7H TIMES.)

2020 FORMAT $(/ / / 15 x, 22$ HINITIAL CONFIGURATION ,I $4 / /(15 x, 12,4 \mathrm{H})$,I5, I 8$) 1$

2021 FORMAT $(/ / 15 X$, OHRATIO IS, F10.6)

2022 FORMAT $(/ / / / / 10 \times, 8$ HMESH IS, I $4 / /)$

2027 FORMAT $(/ / 10 X, 13,21 \mathrm{H}$ MOVES. HAVE BEEN MADE)

2030 FORMAT $(/ / / 15 X, 31$ HRELATIVE MAXIMUM UNDER MESH OF, $14,21 \mathrm{H}$ IS THE CON IFIGURATION//(15X,I2,4H), ,I5, I 8$))$

2032 FORMAT $(/ / / / / 10 X, 19$ HMESH. IS REFINED TO , I 4$)$

2040 FORMAT////15X,41HRELATIVE MAXIMUM WITH FINAL MESH SIZE OF , I4, $21 \mathrm{H}$ $X$ IS. THE CONFIGURATION// $(15 X, 12,4 H)$, I5, I8)

2041 FORMAT $(/ / 15 X, 24 \mathrm{HF}$ INAL VALUF OF RATIO IS ,F10.6)

2042 FORMAT $(/ / 15 \times, 26 H T O T A L$ NUMRER OF MOVES WAS, I $3 / 1 / 1 / 5110 \times, 10 H \times X \times \times \times X X$ $X X X X \| / / / 1 / 1$ END 


\section{References}

[1] E. H. Neville, Solutions of numerical functional equations, Proc. London Math. Soc. 14 (2d ser.), 308-326 (1915).

[2] R. Kerschner, The number of circles covering a set, Am. J. Math. 61, 665 (1939).

[3] S. Verblunsky, On the least number of unit circles which can cover a square, J. London Math. Soc. 24, 164-170 (1949).

[4] L. Fejes Toth, Lagerungen in der Ebene, auf der Kugel, und im Raum, p. 67 (Springer Verlag, 1953).

[5] Gale, On inscribing $n$-dimensional sets in an $n$ dimensional simplex, Proc. Am. Math. Soc. 4, 222-225 (1953).

[6] A. Kolmogoroff, Grundbegriffe der Wahrscheinlichkeitsrechnung, Ergeb. Math. (Berlin), p. 41 (1933).

[7] $\mathrm{R}$. E. Robbins, On the measure of a random set, Ann. Math. Stat. 15, 70-74 (1944).

[8] J. Bronowski and J. Neyman, The variance of the measure of a two-dimensional random set, Ann. Math. Stat. 16, 330-341 (1945).

[9] H. E. Robbins, On the measure of a random set: II, Ann. Math. Stat, 16, 342-347 (1945).

[10] L. A. Santalo, On the first two moments of the measure of a random set, Ann. Math. Stat. 18, 37-49 (1947).

[11] A. Gleason, A search problem in the $N$-cube, Proc. 10 th Am. Math. Soc. Symp. on Applied Math, Vol. 10 (1960).

[12] S. Reiter and G. R. Sherman, Allocating indivisible resources affording external economies or diseconomies, Paper No. 11, Purdue Univ. Inst. for Quantitative Research in Economics and Measurement (Dec. 1960); also, paper No. 13, Choosing an investment program among interdependent projects, by S. Reiter (May 1961).
[13] The Peak-Finding Program, Abstracts of session 43 of the 18th national meeting (Oct. 10-12, 1960) of the Operations Research Society of America. See the Fall 1960 ORSA Bulletin, Supplement 8 to Vol. 8 of Operations Research.

[14] R. C. Buck, Extremal properties of finite sets, delivered at the Symp. on Combinatorial Problems, Princeton, N.J., Apr. 12-13, 1960.

[15] G. E. P. Box and K. B. Wilson, On the experimental attainment of optimum conditions, J. Roy. Stat. Soc. (Ser. B) 13, 1-45 (1951).

[16] G. E. P. Box and J. S. Hunter, Multi-factor experimental designs for exploring response surfaces, Ann. Math. Stat. 28, 195-241 (1957).

[17] S. H. Brooks, A discussion of random methods for seeking maxima, Operations Research 6, 244-251 (1958); A comparison of maximum-seeking methods, Operations Research \%, 430-457 (1959).

[18] D. Hilbert and S. Cohen-Vossen, Geometry and the Imagination, pp. 32-34 (Chelsea, New York, 1952).

[19] E. Landau, Vorlesungen uber Zahlentheorie, Vol. 2.

[20] E. C. Titmarsh, The Lattice-points in a circle, Proc. London Math. Soc. (Ser. 2) 38, 96-115 (1935).

[21] Loo-Keng Hua, The lattice points in a circle, Quarterly J. Math (Oxford Ser.) 13, 18-29 (1942).

[22] H. L. Mitchell III, Numerical experiments on the number of lattice points in a circle, Tech. Rept. 17 Contract Nonr-225(37), (NR-044-211), Applied Mathematics and Statistics Laboratories, Stanford Univ., Calif.

[23] R. Gomory, Outline of an algorithm for integer solutions to linear programs, Bull. Am. Math. Soc. 64, 275 (1958). 